Portland State University

PDXScholar

\title{
The Design and Construction of a Flashlamp- Pumped Frequency-Doubled Dye Laser Applicable to Long Path Monitoring of Air Pollutants
}

Joel Craig Johnson

Portland State University

Follow this and additional works at: https://pdxscholar.library.pdx.edu/open_access_etds

Part of the Applied Mechanics Commons

Let us know how access to this document benefits you.

Recommended Citation

Johnson, Joel Craig, "The Design and Construction of a Flashlamp-Pumped Frequency-Doubled Dye Laser Applicable to Long Path Monitoring of Air Pollutants" (1979). Dissertations and Theses. Paper 2930. https://doi.org/10.15760/etd.2923

This Thesis is brought to you for free and open access. It has been accepted for inclusion in Dissertations and Theses by an authorized administrator of PDXScholar. Please contact us if we can make this document more accessible: pdxscholar@pdx.edu. 
AN ABSTRACT OF THE THESIS OF Joel Craig Johnson for the Master of Science in Applied Science presented

Title: The Design and Construction of a Flashlamp-Pumped FrequencyDoubled Dye Laser Applicable to Long-Path Monitoring of Air Polluţants.

APPROVED BY MEMBERS OF THE THESIS COMMITTEE:
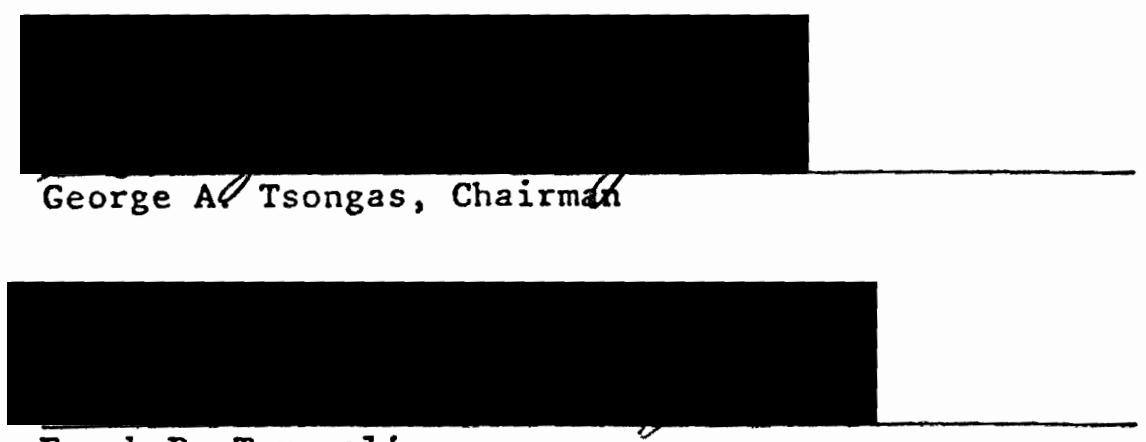

Frank P. Terraglio

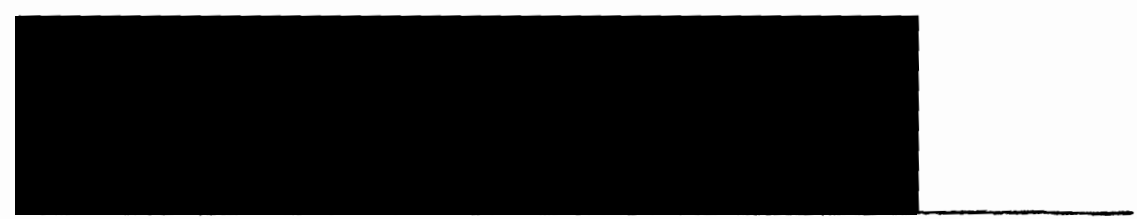

Nan Teh Hsu

The subject of this thesis is the development of a laser light source which could be used in an air pollution monitor to measure the average concentration of sulfur dioxide gas over long paths $(>\mathrm{l} \mathrm{km})$. Many of the problems encountered in designing a long-path pollutant monitor such as instrument cost and reliability, the selection of an optimum spectral region for making the measurement, and light losses due to scattering and turbulence, are considered. The result of these considerations together with a survey of available laser light sources 
suggested that a frequency-doubled dye laser possessed several advantages over other laser light sources.

The construction of a frequency-doubled flashlamp-pumped dye laser was undertaken, and resulted in a laser with a peak output power of $800 \mathrm{~W}$, and which was tunable over the 572 to $610 \mathrm{~nm}$ region with a spectral bandwidth of $0.5 \mathrm{~nm}$. An angle-tuned frequency doubler using an ammonium dihydrogen phosphate (ADP) frequency-doubling crystal was also constructed and converted the dye laser output to tunable ultraviolet with an energy efficiency of $0.3 \%$. This conversion efficiency is in good agreement with theory when the fact that the dye laser spectral width was roughly ten times the spectral acceptance of the frequency doubler is taken into account.

The major problem limiting the usefulness of the frequencydoubled dye laser which was constructed is that of flashlamp lifetime. Several possible solutions to this problem are proposed. 
THE DESIGN AND CONSTRUCTION OF A FLASHLAMP-PUMPED

FREQUENCY-DOUBLED DYE LASER APPLICABLE TO

LONG PATH MONITORING OF AIR POLLUTANTS

by

Joel Craig Johnson

A Thesis Submitted in Partial

Fulfillment of the Requirements for

the Degree of

\author{
MASTER OF SCIENCE \\ in \\ APPLIED SCIENCE
}

Portland State University

1979 


\section{TO THE OFFICE OF GRADUATE STUDIES AND RESEARCH}

The members of the Committee approve the thesis of Joel Craig Johnson presented March 30, 1979.

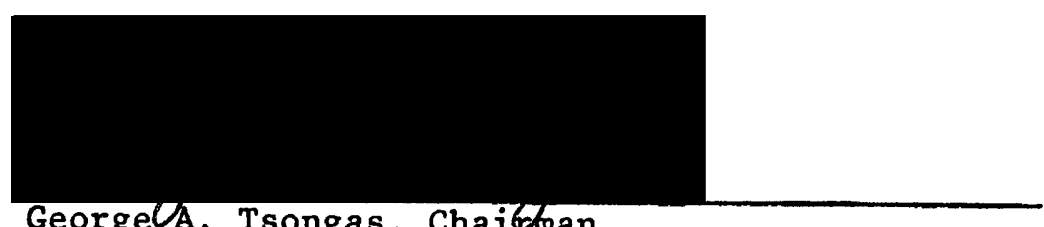

GeorgeVA. Tsongas, Chaibtran
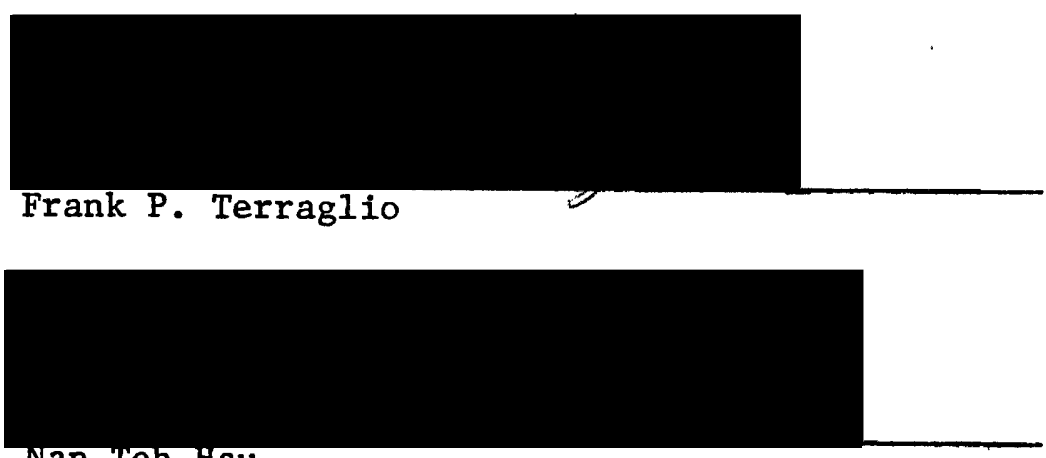

Nan 'Teh Hsu

APPROVED:

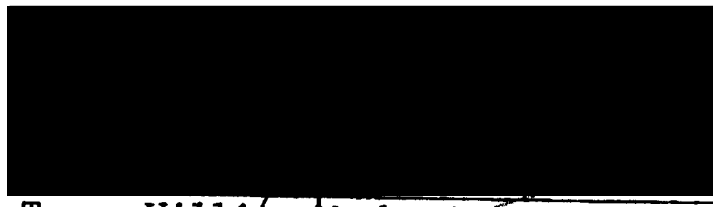

Terry Willi\$, Mechanical Engineering Section Head, Department of Engituering and Applied Science

Stanley Rauch, Dean of Graduate Studies and Research 


\section{ACKNOWLEDGEMENTS}

I would like to first like to thank my major professor, George Tsongas. Without his initial faith in me I would probably not have gone to graduate school. Without his initiative to write the grant which provided the money for this project, this thesis would not exist today. When things were going wrong, and many things did go wrong, George supplied the encouragement and support necessary for a rather green researcher to continue.

Secondly I would like to thank Dr. Frank Terraglio for the good times and conversations which we shared over more cups of Maxwell House instant coffee than I or my stomach wish to recall.

I would like to thank Donna Mikulic for taking the time to just talk.

I would like to thank my family for their understanding and patience when I myself did not understand where I was or what I was doing there. An extension of my immediate family is Eileen Marek who provided me with a great deal of moral support, and to whom I will always be grateful

I would like to thank Dr. Gail Massey for the many conversations which lent technical credibility to this work, and to the Oregon Graduate Center for loaning many of the instruments necessary to complete the measurements on the dye laser and frequency doubler.

The manuscript owes thanks to my typists Bev Kyler, Angeline McClurg, and Karen Moore, and to my graphic artist Deborah Lacy. 
Lastly, I would like to thank God. You know better than

I do your part in this thesis. You have helped me in ways which I do not recognize or understand. I pray that such understanding will come with time.

Thank you all. 


\section{PREFACE}

This work was conceived and begun in the fall of 1971 as an attempt to fill the need for a pollution monitoring instrument which would be useful to local and regional air pollution authorities in performing routine monitoring of average pollutant concentrations. In addition, the pollutant sulfur dioxide was chosen to be the gas of interest, since it was a pollutant of national importance for which no long-path laser monitor had been developed.

A major problem in designing an instrument based upon spectroscopic techniques is the problem of absorption spectra overlap from other materials present in the measurement path. After a substantial literature search into various methods of reducing the effects of such interference, it was decided to adopt the method developed by Barringer Research termed correlation spectroscopy. This method utilizes a number of measurement wavelengths within the pollutant's absorption spectrum in a way to overcome the effects of spectral overlap. A frequency-doubled dye laser was selected as the light source for the instrument since its output fulfilled the two requirements for the light source of tunability and output in the near ultraviolet; and it appeared to offer some attractive advantages such as the possibility of low cost and minimal complexity.

The experimental work for the thesis began in the fall of 1972 and was not completed until the spring of 1976 due to the author's employment. 
TABLE OF CONTENTS

PAGE

ACKNOWLEDGMENTS .................

PREFACE ........................ v v

LIST OF TABLES ...................... $\mathrm{x}$

LIST OF FIGURES . . . . . . . . . . . . . . . xi

CHAPTER

I INTRODUCTION. ..................... 1

II LONG PATH LASER MEASUREMENT CONSIDERATIONS. . . . 12

Interfering Absorption Spectra . . . . . 13

Scattering by Molecules and Particulates... 18

Atmospheric Turbulence . . . . . . . 22

Calculation of Laser Beam Spot Size int

the Absence of Turbulence........ 23

Perturbation of the Laser Beam by

Atmospheric Turbulence. . . . . . . 26

Beam Wander Due to Diurnal, Vertical

Temperature Gradients ........ 33

Methods of Reducing Turbulence Caused Errors - 36

Fluctuation Reduction by Near Simultaneous

Sampling. . . . . . . . . . 36

Fluctuation Reduction by Pulse Averaging. . $\quad 38$

III CHARACTERIZATION OF SPECTRAL REgIONS. . . . . . 41

Vacuum Ultraviolet. . . . . . . . . 41 
Near U1traviolet. . . . . . . . . . .

Visible . . . . . . . . . . . . . .

Near Infrared . . . . . . . . . . 43

Middle Infrared . . . . . . . . . . . 43

Far Infrared. . . . . . . . . . . . 44

Microwave . . . . . . . . . . . . . 44

Sumnary of Spectral Regions.... . . . . 45

IV EXAMINATION OF AVAILABLE TUNABLE LASER SOURCES . 46

Tunable Infrared Laser light Sources. . . . . 47

High Pressure Gas Lasers . . . . . . . . 47

Junction Type Semiconductor Lasers . . . . 48

Parametric Oscillators . . . . . . . 49

Spin-Flip Raman Lasers... . . . . . 50

Summary of Tunable Infrared Laser Devices. $\quad 52$

Tunable Ultraviolet Laser Light Sources . . . 52

Ultraviolet Dye Lasers . . . . . . . 52

Excimer Lasers . . . . . . . . . 52

Frequency-Doubled Tunable Visible Light 53

Sources. . . . . . . . . . . .

Nitrogen Laser Pumped Dye Lasers. . . . 53

Excimer Laser Pumped Dye Lasers . . . $\quad 55$

Flashlamp Pumped Dye Lasers... . . . 55

Choice of Optimum Dye Laser Excitation

Source................ 56

V OVERVIEW OF PROPOSED LONG-PATH MONITOR . . . . . 59 
VI DESIGN AND CONSTRUCTION OF A FLASHLAMP-PUMPED

DYE LASER . . . . . . . . . . . . . . .

Energy Transfer Pathways in Excited State Dye Molecules.............

Analysis and Design of Flashlamp System . .

Flashlamp Driver System . . . . . . . .

81

High Voltage Power Supply. . . . . . . . 81

Energy Storage Capacitor . . . . . . . 83

Triggered Spark Cap. . . . . . . . . 83

Flashlamp Construction . . . . . . . 88

Design of the Dye Cell and Dye Circulation

System. . . . . . . . . . . . .

Dye Cel1 Design and Construction... . . 94

Dye Circulation System . . . . . . . 100

Design of the Laser Resonator . . . . . . . 104

Dye Laser Operation Using a Diffraction Grating as a Tuning Element. . . . . .

Dye Laser Operation Using Flint Prisms as Tuning Elements... . . . . . . . 110

Methods for Producing Simultaneous or Near Simultaneous Outputs at Two Wavelengths...

VII DESIGN AND CONSTRUCTION OF A DYE LASER FREQUENCY DOUBLER. . . . . . . . . . . . . . .

Methods of Phase Matching . . . . . . . . 122

Cholce of Crystal . . . . . . . . . . 127

Frequency Doubling Considerations . . . . . 129

Angle Tolerance of Phase Matching. . . . 129

Walkoff. . . . . . . . . . . 131 
CHAPIER

Spectral Acceptance of an Angle Matched

Frequency Doubler. . . . . . . . 135

Frequency Doubler Fabr1cat1on . . . . . . 136

VIII OPERATION OF THE FLASHLAMP-PUMPED FREQUENCY-

DOUBLED DYE LASER. . . . . . . . . . . . 138

Dye Lager Operating Characteristics . . . . 138

Frequency Doubler Performance . . . . . . . 144

IX CONCLUSIONS AND RECOMMENDATIONS. . . . . . . 148

REFERENCES . . . . . . . . . . . . . . . 152

APPENDIX I . . . . . . . . . . . . . . . . 158 


\section{LIST OF TABLES}

TABLE

PAGE

I Federal Concentration Standards for Five Pollutants .. . . . . . . . . . . . . 42

II Projected Component Costs of Constructing

a Frequency-Doubled Dye Laser . . . . . . . 57 
LIST OF FIGURES

FIGURE

PAGE

1. Envisioned long-path, laser pollutant monitor system. . . . . . . . . . . . . . . . . .

2. Light projecting system such as might be used in a long-path pollutant monitor utilizing a conventional light source.... . . . . . .

3. Light transmission loss due to Rayleigh scattering as a function of wavelength. . . . . . .

4. Light transmission loss due to Mie scattering as a function of wavelength, and for three visibility conditions . . . . . . . . . . . .

5. Minimum receiver/transmitter diameter as a function of pathlength and for three wavelengths... . . . . . . . . . . . . .

6. Schematic representation of the perturbation of a plane wave propagating through a turbulent medium . . . . . . . . . . . . . . . .

7. Minimum receiver size necessary. to capture 99 percent of the transmitted laser power 36 percent of the time under best and worst case atmospheric beam steering conditions. . . . .

8. Receiver diameter necessary to collect 95 percent of the transmitted laser power under high turbulence beam spreading conditions. . . . .

9. Block diagram of the proposed.long path $\mathrm{SO}_{2}$ monitor............... . . .

10. Low resolution absorption spectrum of sulfur dioxide gas in the middle ultraviolet . . . .

11. High resolution absorption spectrum of sulfur dioxide gas in the middle ultraviolet . . . .

12. Block diagram of detection electronics. .... 
13. Block diagram of a frequency-doubled, flashlamp-pumped dye laser . . . . . . . . .

14. Simplified rotational mount to be used to automatically rotate tuning components. . . .

15. Absorption and emission spectrum of rhodamine 6G laser dye. . . . . . . . . . . . .

16. Dye energy levels and transitions relevant to an organic dye laser. . . . . . . . . . .

17. Simplified diagram of a capacitor discharge powered flashlamp pumping system.

18. Simplified flashlamp pumping system showing only those components and parameters used in estimating the flashlamp pulse length .....

19. Simplified schematic of the high voltage power supply used to charge the energy storage capacitor . . . . . . . . . . . .

20. Diagram of the triggered spark gap used to fire the flashlamp..............

21. Electrical breakdown characteristics of the triggered spark gap ............

22. Simplified schematic of the circuit used to trigger the triggered spark gap.......

23. Diagram of the low pressure air filled flashlamp. . . . . . . . . . . .

24. Diagram of the one atmosphere, flowing argon flashlamp . . . . . . . . . . . .

25. Flashlamp light output as a function of time. .

26. Cross-sectional diagram of the elliptical dye cell cavity showing the placement of the dye cell and flashlamp. ...........

27. Broadband dye laser output energy as a function of input energy to the flashlamp. .......

28. Dye laser tuning configuration using a Littrow mounted diffraction grating . . . . . . . 
29. Dye laser tuning configuration using two prisms as tuning elements... . . . . . . 106

30. Tuning prism showing minimum deviation condition. . . . . . . . . . . . . . 111

31. Dye laser tuning configuration capable of providing simultaneous outputs at two different wavelengths . . . . . . . . . .

32. Dye laser tuning scheme utilizing a fast beam deflector to tune the laser between two closely spaced wavelengths. . . . . . . . . . . .

33. A crystalline electro-optic beam deflector. .

34. Crystalline axis showing the indices of refraction seen by various incident electric fields. . . . . . . . . . . . . . . .

35. Indices of refraction for the non-linear crystal ammonium dihydrogen phosphate.....

36. Phase matched wavelength as a function of angle for the crystal ammonium dihydrogen phosphate . . . . . . . . . . . . . . .

37. Frequency doubling in an angle matched crystal illustrating walkoff between the fundamental and second harmonic beams ...........

38. Final operating configuration of flashlamppumped frequency-doubled dye laser. . . . . .

39. Rhodamine $6 G$ dye laser output energy as a function of wavelength. . . . . . . . . . .

40. Integrating photodiode circuit used to measure dye laser output energy . . . . . . . . . . .

41. Dye laser output pulse. . . . . . . . . .

42. Second harmonic output energy as a function of wavelength. . . . . . . . . . . . . . . 
CHAPTER I

\section{INTRODUCTION}

At the present time approximately forty gaseous air pollutants are being monitored in the United States, primarily using so-called wet chemical techniques. ${ }^{1}$ With these techniques a sample of air containing the pollutant is bubbled through a solution which chemically traps the pollutant. After a predetermined amount of sample gas has passed through the absorbing solution, the solution is chemically analyzed to determine the amount of pollutant trapped. By knowing the amount of pollutant gas trapped and the volume of gas from which the pollutant was trapped, it is possible to calculate the original concentration of the pollutant.

While wet chemical techniques have been and currently are widely used, they do have a number of limitations. ${ }^{2}$ Frequently the chemical technique used to detect the presence of the pollutant gas in the absorbing solution is an insensitive one, thus requiring that substantial anounts of the pollutant gas be trapped by the absorbing solution before an accurate analysis can be made. This requirement, when coupled with the typical trace pollutant concentration levels found in the atmosphere, implies that large volumes of air must be drawn through the absorbing solution. Due to the limited flow rates of most sampling systems, large volumes of air may be translated to mean long sampling times. Indeed, sampling times on the order of several hours are not uncommon. Such sampling times are unacceptably 
long for a number of gaseous pollutants in that the Federal Primary Standards ${ }^{3}$ specify the maximum concentration permissible in any single hour. Thus it is essential that detection instruments be developed which do not require long sampling times. In fact, it would be highly desirable to develop an instrument capable of real-time pollutant monitoring.

A second major problem is that in many instances, the chemical method used to determine the concentration of a particular pollutant has also been found to be sensitive to a variety of other pollutants a1so present in the atmosphere. For this reason it is often necessary to precondition the sample gas before passing it through the absorbing solution. Preconditioning refers to some method of either removing or chemically altering those gases which would later interfere with the determination of the pollutant concentration. The effects of such preconditioning processes upon the pollutant of interest can often leave the results of any subsequent concentration analysis open to question.

While a wide variety of new instruments have been developed to alleviate the aforementioned shortcomings of wet chemical methods, 4 one very important characteristic remains in that almost all such instruments developed so far have been point measurement devices. That is to say, the concentration value determined by the instrument is indicative only of the pollutant concentration at the point from which the sample was drawn. In many instances, such as source monitoring, the only information desired is the concentration of a particular pollutant at some point. In many other cases however, it is desirable 
to monitor the average concentration of a pollutant over a geographically large area such as a city. At the present time an area-wide program might entail the use of a large number of point monitors digtributed throughout the study area. The cost of acquiring and maintaining a large number of point monitors is, in most cases unacceptably high. A more practical solution would be a single instrument which could replace all or a number of the point monitors.

Indeed, in the last few years a great deal of interest has been shown in the development of so-called long-path monitors. In a longpath monitor, light of known intensity is passed through the polluted atmosphere to a detector where the transmitted light intensity is monitored (see Fig. 1). In a polluted atmosphere, some of the light will be absorbed by the various pollutants. If one is clever enough to pick a wavelength for which only one pollutant gas absorbs, then the amount of absorption is indicative of the amount of pollutant present between the light source and the detector. The functional relationship between the pollutant concentration and transmitted light intensity is Beer's law: 5

$$
I=I_{0} e^{-a(\lambda) c L}
$$

where:

I = the transmitted 1 ight intensity

$I_{0}=$ the initial light intensity

$a(\lambda)=$ the pollutant's absorption coefficient at the wavelength $\lambda$ of interest

$c=$ the average pollutant concentration along the light path 

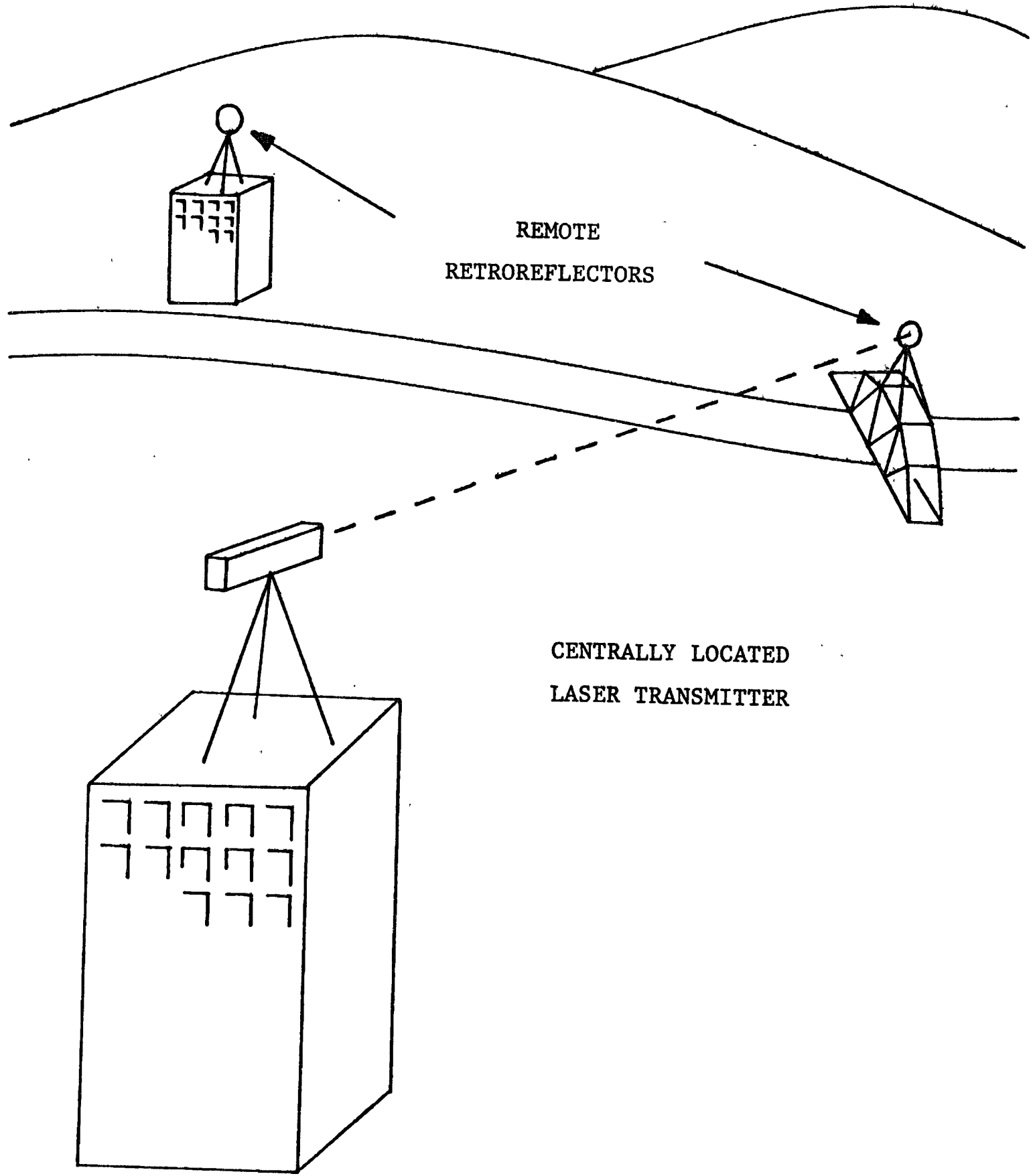

CENTRALLY LOCATED

LASER TRANSMITTER

Fig. 1. Envisioned long path, laser pollutant monitor system. 
$L=$ the length of the absorption path over which the light travels

To illustrate the sensitivity which might be expected from a long-path monitor, consider an instrument designed to measure the concentration of sulfur dioxide gas using ultraviolet light. Solving (1.1) for the pollutant concentration $c$ one gets:

$$
c=\frac{-1}{\mathrm{aL}} \ln \left(\frac{\mathrm{I}}{I_{0}}\right)
$$

Assume that the instrument operates over a $1 \mathrm{~km}$ path and is capable of detecting a change in light intensity: due to absorption of 0.5 percent. The absorption coefficient for sulfur dioxide around $300 \mathrm{~nm}$ is on the order of $10^{3} \mathrm{~atm}^{-1} \mathrm{~m}^{-1}$. Inserting these values into (1.2) one finds that the minimum detectable concentration is on the order of 5 partsper-billion.

This minimum concentration is a factor of 6 less than the maximum yearly average concentration for $\mathrm{SO}_{2}$ as established by the Federal government and thus represents a useful signal-to-noise ratio. Early attempts at making long-path monitoring devices utilized light sources either in the form of a hot filament or a high current electric arc. Unfortunately, such light sources produce light which is intrinsically unsuitable for making long-path absorption measurements. The problems are two-fold. First, while the arc or filament may radiate a large amount of light, the amount of power within any narrow wavelength interval is sma1l. Second, the light is emitted in all directions rather than in a collimated beam such as would be desired for use in a long path monitor.

To illustrate the seriousness of these two problems, consider 
an optical system in which light from an arc is collected by a lens and is focussed on a detector such as illustrated in Fig. 2. To determine how much light will be incident upon the detector, we must first know how large the light source appears to be in the detector plane. The 1ateral magnification of the light source in the image or detector plane is known from geometrical optics to be: ${ }^{7}$

$$
M=\frac{L}{d}
$$

where:

$$
\begin{aligned}
& M=\text { the lateral magnification } \\
& L=\text { the lens to image distance } \\
& d=\text { the source to lens distance. }
\end{aligned}
$$

The maximum useful image size of the light source will be the same as that of the receiver collector. Relaying the receiver collector size back through the magnification of the projection optics we arrive at the maximum useful size of the light source:

$$
r_{s}=\frac{r_{c}}{M}=r_{c} \frac{d}{L}
$$

where:

$$
\begin{aligned}
& r_{s}=\text { the maximum useful radius of the emitting arc } \\
& \text { or filament assuming a circular collector } \\
& r_{c}=\text { the radius of the receiver collector } \\
& d=\text { the light source to projection lens distance } \\
& L=\text { the projection lens to receiver distance. }
\end{aligned}
$$

The maximum usable light source area is then:

$$
A_{s}=\pi\left(\frac{d}{L}\right)^{2} r_{c}^{2}
$$

where $A_{S}$ is the area of the light source whose light strikes the 


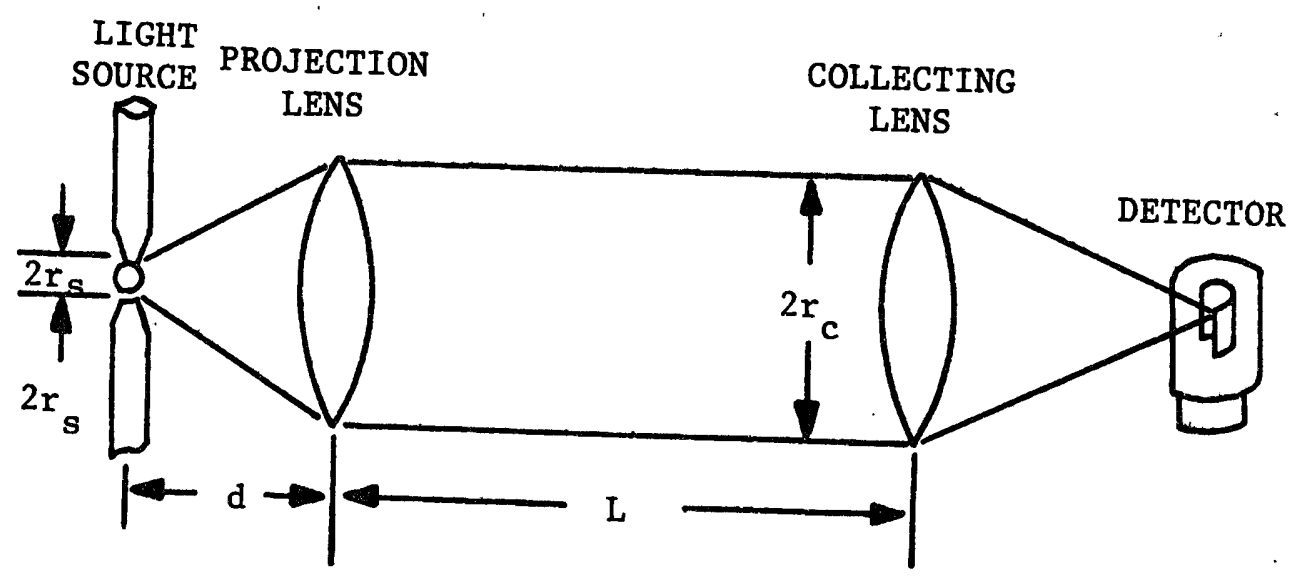

Fig. 2. Light projecting system such as might be used in a long- 
receiver. Quick examination of (1.5) reveals that the size of the light source will be quite small. If the lamp is assumed to radiate as a blackbody as many arcs and most hot filaments do, then the amount of power emitted per square centimeter of emitter surface area, per Angstrom of wavelength per steradian of solid angle will be given by : 8

$$
R(\lambda, T)=\frac{3.74 \times 10^{15}}{\lambda^{5}\left[\frac{1.438 \times 10^{7}}{\lambda T}-1\right]}
$$

where:

$$
\begin{aligned}
& R=\text { the spectral radiance in wat } \mathrm{s} / \mathrm{cm}^{2} / \AA / \text { steradian } \\
& \lambda=\text { the wavelength in nanometers for which the } \\
& \text { radiance is desired } \\
& T=\text { the source temperature in degrees Kelvin. }
\end{aligned}
$$

The amount of power withit any one Angstrom wavelength interval, which can be collected and imaged on a receiver is given by the products of (1.5) and (1.6) and one final factor $\Omega$ which corresponds to the solid angle of the projection optics from the source.

$$
\begin{aligned}
P_{R} & =A_{s} R(\lambda, T) \Omega \\
& =r_{c}^{2}\left(\frac{d}{L}\right)^{2}\left[\frac{3.74 \times 10^{15}}{\left(\frac{1.438 \times 10^{7}}{\lambda T}-1\right)_{\lambda}}\right]\left[2 \pi 1-\left[1-\left(\frac{d}{2 r_{P}}\right)^{2}\right]^{1 / 2}\right]
\end{aligned}
$$

where the units of $A_{s}$ must be square centimeters. Let us now evaluate the above expression for two cases of interest: first for longpath monitoring in the ultraviolet at $300 \mathrm{~nm}$, and second in the middle infrared at 5 microns. The reasons for choosing these spectral regions 
will become clear in chapter III.

Let us assume that both the projecting and receiving optics are mirrors $15 \mathrm{~cm}$ in diameter and have $15 \mathrm{~cm}$ focal lengths. Mirrors are a better choice than lenses since for comparable diameters and focal lengths they are physically lighter and less expensive, and $15 \mathrm{~cm}$ represents a compromise between size and cost. Furthermore, let us assume that the transmitter and collector are separated by one kilometer. The highest temperature lamp commercially available, and therefore the one which produces the greatest amount of power per wavelength interval, operates at a blackbody temperature of $10,000 \mathrm{~K} .{ }^{9}$ Inserting these values into (1.7), and assuming a wavelength of $300 \mathrm{~nm}$, we get a received power of $0.5 \mu \mathrm{W}$. If this were incident upon a photodetector such as a 1 P28 photomultiplier; ${ }^{10}$ it would give an electrical output only ten times greater than the noise output from the detector in the absence of any signal. Since many other lossy optical components such as lenses, prisms, as we11 as atmospheric transmission losses would be present in a real system, such a small optical signal would generally not be acceptable.

In the infrared at 5 microns, the amount of received power would be $1.5 \times 10^{-10}$ W. If this light were chopped at a $1 \mathrm{kHz}$ frequency to allow AC detection and amplified by an amplifier with a 10 percent of center frequency bandwidth, the output from a cooled, $1 \mathrm{~mm}^{2} \mathrm{PbSe}$ detector ${ }^{11}$ would be just larger than the detector noise.

Thus, because of their spectrally broadband emission, and poor collimation characteristics, conventional light sources are not well suited for use in long-path monitoring instruments. 
Laser light sources, however, intrinsically produce well collimated beams which are spectrally very narrow with linewidths of $1 \AA$ or less and divergences of 1 milliradian or less being typical. Byers $^{12}$ has stated that using a laser light source, it should be possible to make pollutant concentration measurements over pathlengths as long as $50 \mathrm{~km}$. Thus the laser would appear to be the missing link needed for the realization of a true long-path monitor. In fact, a number of long-path research instruments have recently been built for the detection of a variety of pollutant gases. ${ }^{13-16}$

Those long-path laser instruments which have been developed thus far, have displayed adequate sensitivity and a few are capable of monitoring more than one pollutant. None of those instruments, however, have had the simplicity, the reliability, and especially the low cost to be valuable to air pollution control agencies. This is evidenced by the comnercial nonavailability of a laser-based long-path pollutant monitor. The need remains, therefore, for a long-path detection instrument which would be suitable for use in the field by local and regional air pollution authorities.

It was thus decided to try to develop a laser-based, long-path pollutant monitor which could be low in cost, reliable in operation, require little maintenance, and capable (ideally) of monitoring a number of the major gaseous air pollutants found in urban atmospheres. While it is obviously advantageous to be able to monitor a number of pollutants using the same instrument, it was decided to initially. concentrate on developing an instrument for monitoring sulfur dioxide. The reasons for choosing sulfur dioxide were that it is a pollutant 
of national interest for which federal standards had been established, and it was a pollutant for which no laser long-path monitor existed at the inception of this work. 16 
CHAPTER II

LONG-PATH LASER MEASUREMENT CONSIDERATIONS

One of the primary considerations in constructing any measurement instrument is to make certain that the quantity to be measured is the only quantity being measured. That is to say, if one is making an instrument to measure the resistance of a wire, then the instrument should not be subject to exrors introduced by the leads used to connect the measurement instrument to the wire under test. Or, if one is making an instrument to measure the amount of light absorbed by some medium, then precautions must be taken to ensure that the measurement is not influenced by absorption or scattering from anything but the medium of interest. It is to the understanding of this general problem that chapter II is dedicated, for a light beam passing through the atmosphere may be subjected to a number of processes which can produce errors in the absorption measurement if not accounted for and corrected. In particular, a light beam being propagated through the atmosphere may be absorbed by gases other than the pollutant of interest. A portion of the light beam may be scattered by particulates in the atmosphere and even the air molecules themselves. In addition, atmospheric temperature variations along the path can cause the beam to spread and wander about the receiver plane. Such beam movements can result in a loss of power on the receiver which results in measurement errors.

Each of these effects will be examined in this chapter. 


\section{Interfering Absorption Spectra}

The first problem to be examined will be that of interference from gases other than the pollutant, whose absorption spectra overlap in wavelength the absorption spectra of the pollutant gas. If one makes a simple, single wavelength absorption measurement such as described in the introduction, it will be impossible to tell how much of light was absorbed by the pollutant, and how much was absorbed by any interfering gases. The reason can be easily seen by examining the Beer's law expression for absorption by two gases:

$$
I=I e^{-\left[a_{1}\left(\lambda_{1}\right) c_{1}+a_{2}\left(\lambda_{2}\right) c_{2}\right] L}
$$

where:

$$
\begin{aligned}
& \text { I }=\text { the received light intensity } \\
& I_{0} \quad=\text { the transmitted light intensity } \\
& a_{1}\left(\lambda_{2}\right)=\text { the absorption coefficient of the pollutant } \\
& \text { of interest at the wavelength } \lambda_{1} \\
& c_{1}=\text { the concentration of the pollutant } \\
& a_{2}\left(\lambda_{2}\right)=\text { the absorption coefficient of the interfering } \\
& \text { gas at the wavelength } \lambda_{1} \\
& c_{2}=\text { the interfering gas concentration } \\
& \text { I = the pathlength. }
\end{aligned}
$$

This can be rewritten as:

$$
\ln \frac{I(\lambda)}{I_{0}(\lambda)}=-a_{1}\left(\lambda_{1}\right) c_{1} L-a_{2}\left(\lambda_{1}\right) c_{2} L
$$

which is nothing more than a linear equation in at least two unknowns, $c_{1}$ and $c_{2}$. In general at least two independent equations will be required to determine $c_{1}$, the concentration of the pollutant of interest. 
The second independent equation can be obtained by performing the absorption measurement at a different wavelength $\lambda_{2}$ such that:

$$
\ln \frac{I\left(\lambda_{2}\right)}{I_{0}\left(\lambda_{2}\right)}=-a_{1}\left(\lambda_{2}\right) c_{1} L-a_{2}\left(\lambda_{2}\right) c_{2} L .
$$

Solving (2.3) for $c_{2}$ and using that expression in (2.2) we can solve for the pollutant concentration:

$$
c_{1}=\frac{\left(\frac{a_{2}\left(\lambda_{1}\right)}{a_{2}\left(\lambda_{2}\right)}\right) \ln \left(\frac{I\left(\lambda_{2}\right)}{I_{0}\left(\lambda_{2}\right)}\right)-\ln \left(\frac{I\left(\lambda_{1}\right)}{I_{0}\left(\lambda_{1}\right)}\right)}{a_{1}\left(\lambda_{1}\right) L\left[1-\left(\frac{a_{1}\left(\lambda_{2}\right)}{a_{1}\left(\lambda_{1}\right)}\right)\left(\frac{a_{2}\left(\lambda_{1}\right)}{a_{2}\left(\lambda_{2}\right)}\right)\right]}
$$

The previous analysis could have been performed for the general case of $\mathrm{n}$ interfering gases by making $\mathrm{n}$ measurements at $\mathrm{n}$ different wavelengths. Usually little is known about the values of the absorption coefficients of the interfering gas, thus making it impossible to evaluate (2.4) for $c_{1}$. However it is sometimes possible to pick two wavelengths $\lambda_{1}$ and $\lambda_{2}$ such that there is a significant change in the absorption coefficient of the gas of interest, but little change in the absorption coefficient of any interfering gas. If $a_{2}\left(\lambda_{1}\right) \sim a_{2}\left(\lambda_{2}\right)$, then $(2.4)$ can be reduced to:

$$
c_{1}=\frac{\ln \left(\frac{I\left(\lambda_{2}\right)}{I_{0}\left(\lambda_{2}\right)}\right)-\ln \left(\frac{I\left(\lambda_{1}\right)}{I_{0}\left(\lambda_{1}\right)}\right)}{\left(a_{1}\left(\lambda_{1}\right)-a_{1}\left(\lambda_{2}\right)\right) L} .
$$

The absorption coefficient of a gas is generally a slowly varying function of wavelength except in the vicinity of the center of an absorption line, where under conditions normally found near the surface of the earth, the strength of the absorption line will have a rapidly 
varying Lorentzian shape with respect to wavelength. ${ }^{17}$ The width of the Lorentzian absorption 1 ine will depend upon parameters particular to the gas and absorption line of interest. To make the absorption coefficient for the interfering gas at the two measurement wavelengths as similar as possible it is desirable to keep the two measurement wavelengths as close together in wavelength as possible; the minimum wavelength separation might be taken to be one-half of the pollutant gase's absorption linewidth. Making the first measurement at the wavelength of maximum absorption for the pollutant gas and allowing the second measurement wavelength to fall one-half absorption linewidth away, also tends to maximize the change in the pollutant's absorption coefficient, a condition for high sensitivity.

Measurement systems which rely upon the fact that the value of the absorption coefficient of the interfering gas at the two measurement wavelengths is constant are termed dual beam systems. 5 Such systems have long been used in the laboratory to subtract out interfering backgrounds. The dual beam system is capable of discriminating against forms of spectral interference which result in a loss of transmitted 1 ight which does not vary significantly with wavelength, and can therefore also be used to eliminate light losses due to scattering.

If the measurement is subject to interference from a gas with a rapidly varying interference spectrum, it is necessary to adopt a measurement method utilizing many wavelengths. One could think of a brute force system using a laser which emits light at a large number of wavelengths, and a computer to solve a series of linear equations 
to determine the concentrations of the various absorbers present. A small system based on this idea was built by General Electric ${ }^{18}$ and used four wavelengths from an infrared carbon dioxide laser. It was capable of measuring ammonia and ethylene gas at part-per-billion concentrations over a two mile range. Unfortunately ethylene and ammonia are not pollutants of extreme interest. Had it been possible to match the output wavelengths of the $\mathrm{CO}_{2}$ laser to one of the more interesting pollutants such as sulfur dioxide or carbon monoxide, the instrument would have been useful. Unhappily, coincidences between pollutant absorption lines and the output from discrete wavelength lasers such as $\mathrm{CO}_{2}$ are rare.

A second type of multiple wavelength, pollution monitoring instrument is the correlation spectrometer ${ }^{19}$ developed by Barringer Research, Ltd., Ontario, Canada. In this instrument, light from a blackbody light source such as the sun or arc lamp, which has passed. through the atmosphere enters the correlation spectrometer where it is dispersed by a diffraction grating or prism. Detection is accomplished by inserting a mask in the spectrometer's focal plane, which allows only light at those wavelengths corresponding to the pollutant's absorption lines to pass through to the detector. If the mask oscillates in the focal plane of the spectrometer such that at one point in its physical excursion, only those wavelengths corresponding to the pollutant absorption lines are allowed to pass, while at some later time in the mask vibration the light passing through the mask corresponds to wavelengths for which little pollutant absorption occurs, it is possible to perform dual wavelength measurements at a number of pollutant 
absorption lines simultaneously. The number of measurement wavelengths is determined by the number of slits in the mask, and for sulfur dioxide gas is usually on the order of five to ten slits. The ability of the correlation spectrometer to discriminate against interference stems purely. from the fact that it is improbable that an interfering gas will have an absorption spectra which exactly matches the pollutant's absorption spectrum at all of the measurement wavelengths. While it is possible to imagine that a number of interfering absorption spectra might synergistically combine in a way which could fool this system, no evidence to this effect has been found.

The fact that this instrument uses a blackbody light source may at first appear inconsistent with earlier arguments which showed that because of the small amount of optical power which would be incident upon an instrument's photodetector, blackbody light sources were a poor choice for a long-path monitor. The correlation spectrometer is able to avoid the low signal problem by using many slits instead of one, and by allowing each of the slits to pass many Angstroms of optical power. The penalty for making the slits wide is a reduction in the instrument's sensitivity to a particular pollutant and in its ability to discriminate against interference. Since lasers intrinsically produce light with a very narrow linewidth, a correlation spectrometer which uses a laser light source should display superior sensitivity and selectivity to one which uses a blackbody light source.

While the Barringer instrument is limited to detecting a single pollutant because of the necessity of using a mask which matches the pollutant's absorption spectrum, it is capable of 
detecting any gas possessing rapidly varying absorption spectra lying within the wavelength range of the spectrometer, by simply changing masks. With a tunable laser light source the through-put advantage would not be needed and the wavelength selecting function of the mask could be simulated by sequentially tuning the laser to the appropriate wavelengths. Since the tuning function can be controlled by a small computer programmed to select any number of desired wavelengths, it should be possible to monitor several pollutants by tuning the laser to the appropriate wavelengths.

Thus at least two methods utilizing two or more wavelengths have been developed to reduce if not eliminate the effects of spectral overlap in absorption measurements.' Both can be used with laser sources. Which method is adopted will depend upon the severity of the interference problem encountered, and the laser light source used.

\section{Scattering by Molecules and Particulates}

The second general problem, that of scattering from molecules and particulates can be divided into two regimes depending upon the the size of the scatterer relative to the wavelength of the light being propagated. When the wavelength is much larger than the size of the scatterer, the process is termed Rayleigh scattering ${ }^{20}$ and has a functional dependence which is inversely proportional to the fourth power of the wavelength, i.e.:

$$
0 \propto \frac{1}{\lambda^{4}}
$$

where:

$$
\sigma=\text { the Rayleigh scattering coefficient }
$$


$\lambda=$ the wavelength of the 1 ight.

The fractional transmission for a one kilometer path through air is shown in Fig. $3 .^{20}$ As can be seen, for $1 \mathrm{~km}$ paths Rayleigh scattering becomes an almost negligible loss mechanism at wavelengths longer than about $320 \mathrm{~nm}$, the 90 percent transmission point.

When the size of the scattering particle is on the same order, or larger than the wavelength of the light being scattered, the scattering process is termed Mie scattering, 20 and the amount of 1 ight scattered will depend upon the wavelength of the light being propagated, the size spectrum of the scattering particles, and the index of refraction of the particles. The fraction of light transmitted as a function of wavelength under various visibility conditions is shown in Fig. 4. 21 Once again, the transmission decreases rapidly as one goes to short wavelengths. Neither form of scattering is so intense as to preclude the possibility of making a long path measurement provided there is sufficient light available from the transmitter to overcome scattering losses. At ultraviolet wavelengths around $300 \mathrm{~nm}$, the transmitter will have to overcome losses of the order of 75 percent. In the middle infrared, the losses are a factor of three smaller or about 25 percent under medium haze conditions.

In addition to Mie and Rayleigh scattering, fog will reduce the intensity of a transmitted laser beam. At wavelengths less than roughly 10 microns the amount of 1 ight transmitted through fog will be on the order of one part in $10^{14} \cdot 20$ At longer wavelengths the transmission improves slowly, but in general, optical measurements through fog will not be possible. 


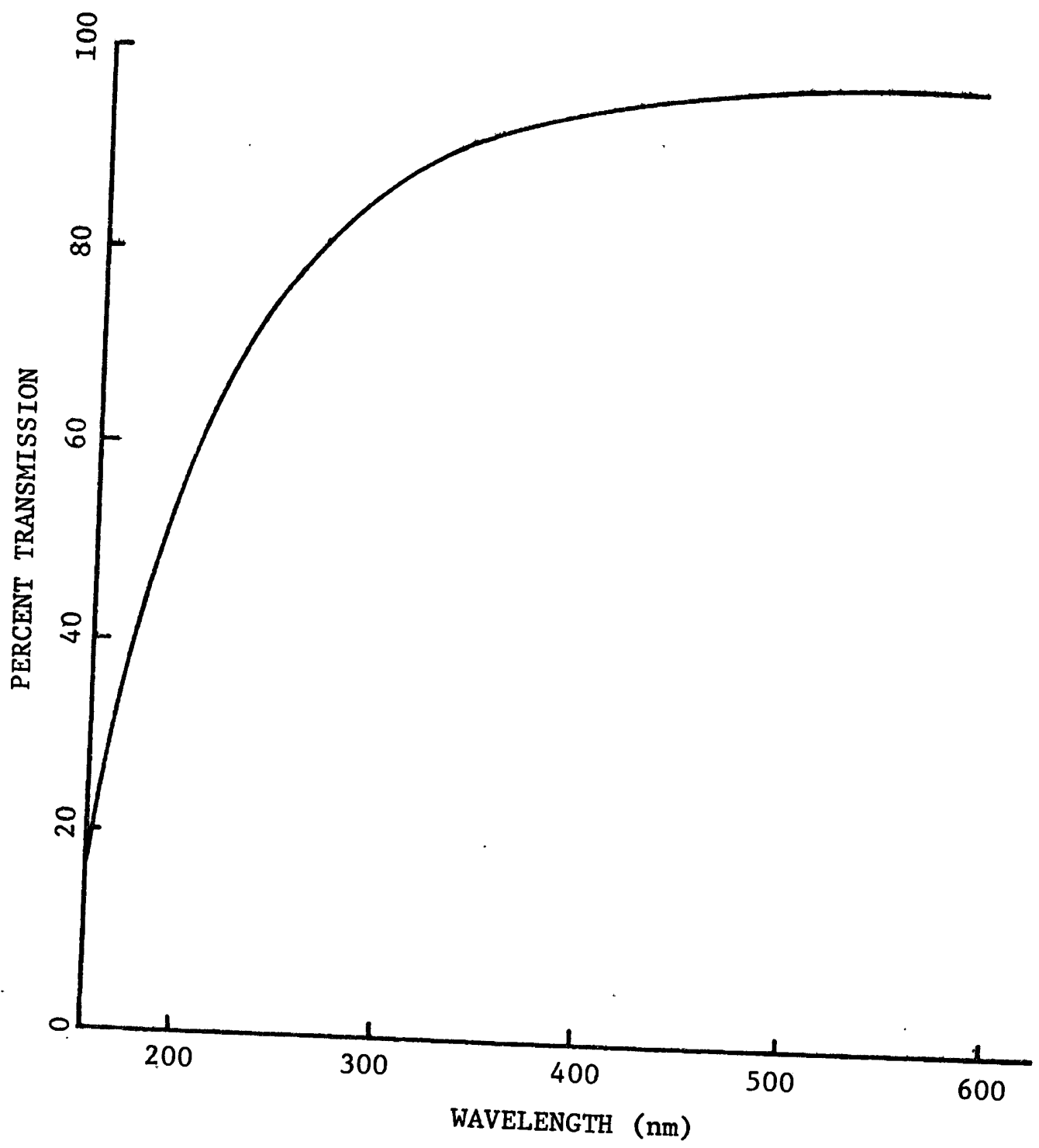

Fig. 3. Atmospheric transmission over a $1 \mathrm{~km}$ sea level path 


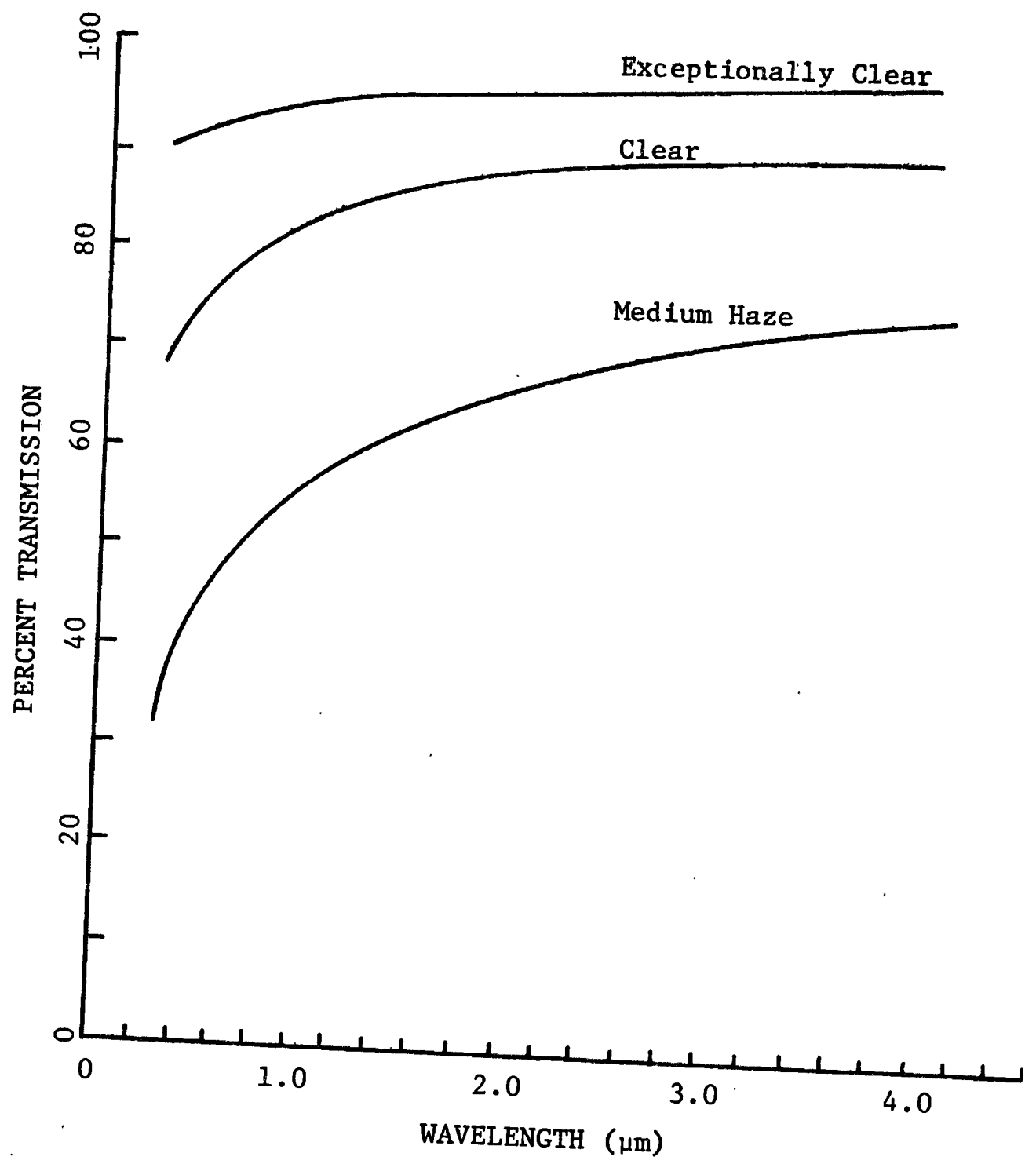
Fig. 4. Measured atmospheric transmission over a $1 \mathrm{~km}$ sea level
path showing losses due to Mie scattering. (Ref. 21) 
Atmospheric Turbulence

The third general problem to be faced is that of atmospheric turbulence. The general problem of light propagation through a turbulent atmosphere is beyond the scope of this thesis, and the reader is referred to any one of the excellent review papers on this subject for more detailed analysis. 21,22 simplistically, atmospheric turbulence refers to the fact that the atmosphere is not homogeneous with respect to temperature but rather consists of various sized "bubbles" of air which are warmer or cooler than the surrounding air. Since the refractive index of air depends upon the temperature of the air, the bubbles of warmer or cooler air act like lenses of varying focal length which are carried through the beam by the wind. 23 The effect of such "lenses" upon the beam is simplistically two-fold. "First, if the diameter of the "lenses" is smaller than the beam, a case corresponding to very small regions of warm or cold air, then the primary result will be to cause the beam to spread in the receiver plane. If on the other hand the "lenses" are large in diameter relative to the diameter of the beam, a case corresponding to very large bubbles of warm or cold air, then the main effect will be that the beam will wander around in the receiver plane. Since the atmosphere has in it a virtual continuum of bubble sizes, both beam spreading and steering will be present. The net effect of both phenomena is to increase the required size of the receiver collector since it must now capture not just a stationary beam of a certain size, but one which both "blooms" due to lensing, and moves around in the receiver plane because the "lenses" are moving. In a following section we will calculate the size of a receiver 
collector necessary to capture a laser beam in the presence of turbulence. If the entire bearn can be captured then the effects of turbulence will be zero since energy will be conserved. As will be seen, the size of a collector necessary to capture the entire beam will become prohibitively large under worst case turbulence conditions and it will be necessary to adopt some method other than just using a larger collector to reduce the effects of turbulence. Before proceeding with such calculations it is necessary to determine the diameter of the collector which could capture the entire beam in the absence of turbulence. This collector size can then be used to calculate the necessary collector diameter in the presence of turbulence.

\section{Calculation of Laser Beam Spot Size in the Absence of Turbu-}

lence. The diameter of a laser beam is not a parameter which can be measured in the same manner as one would the size of a box or a desk, since a laser beam has no sharply defined edge beyond which there is no light. In genera1, the intensity of a laser beam gradually approaches zero as one moves away from the beam axis and is usually characterized as having a Gaussian intensity profile. ${ }^{24}$ Because a laser beam lacks a well defined edge, its size is usually measured in terms of a parameter which is termed the spot size or radius, and is defined in the case of a beam with a Gaussian intensity distribution to be the distance from the beam axis to a point where the laser beam intensity has dropped to $1 / \mathrm{e}^{2}$ of its on-axis value.

The beam spot size $w$ is in turn a function of the distance along the beam axis measured from a plane where the radius of 
of curvature of the beam wave front is infinite. This plane is termed the waist. In geometrical optics this plane was called the focal plane, and for laser beams which are slowly convergent or divergent, the two planes are equivalent. The beam spot size $w$ is given by: 24

$$
w(z)=w_{0}\left[1+\left(\frac{\lambda z}{\pi w_{0}^{2}}\right)^{2}\right]^{1 / 2}
$$

where:

$$
\begin{aligned}
w(z)= & \text { the beam spot size (radius) a distance } z \\
& \text { away from the beam waist } \\
\lambda \quad= & \text { the laser wavelength } \\
w_{0}= & \text { the beam spot size at the beam waist, } z=0 .
\end{aligned}
$$

As is shown in Appendix I, the smallest diameter which can be propagated between two points a distance $\mathrm{L}$ apart is one for which the spot size $w$ at the two ends of the path is equal and related to the spot size in the waist region by:

$$
w=w_{0} \sqrt{2}
$$

which can be rewritten as:

$$
\mathrm{w}=\left(\frac{\lambda L}{\pi}\right)^{1 / 2}
$$

A laser beam which is propagated in this manner is termed confocally focussed. To capture 99 percent of the transmitted power, both the transmitter and receiver should have a diameter of $3 w,{ }^{25}$ and this value of receiver/transmitter diameter is plotted in Fig. 5 as a function of pathlength for three wavelengths. As can be seen, the beam sizes are 


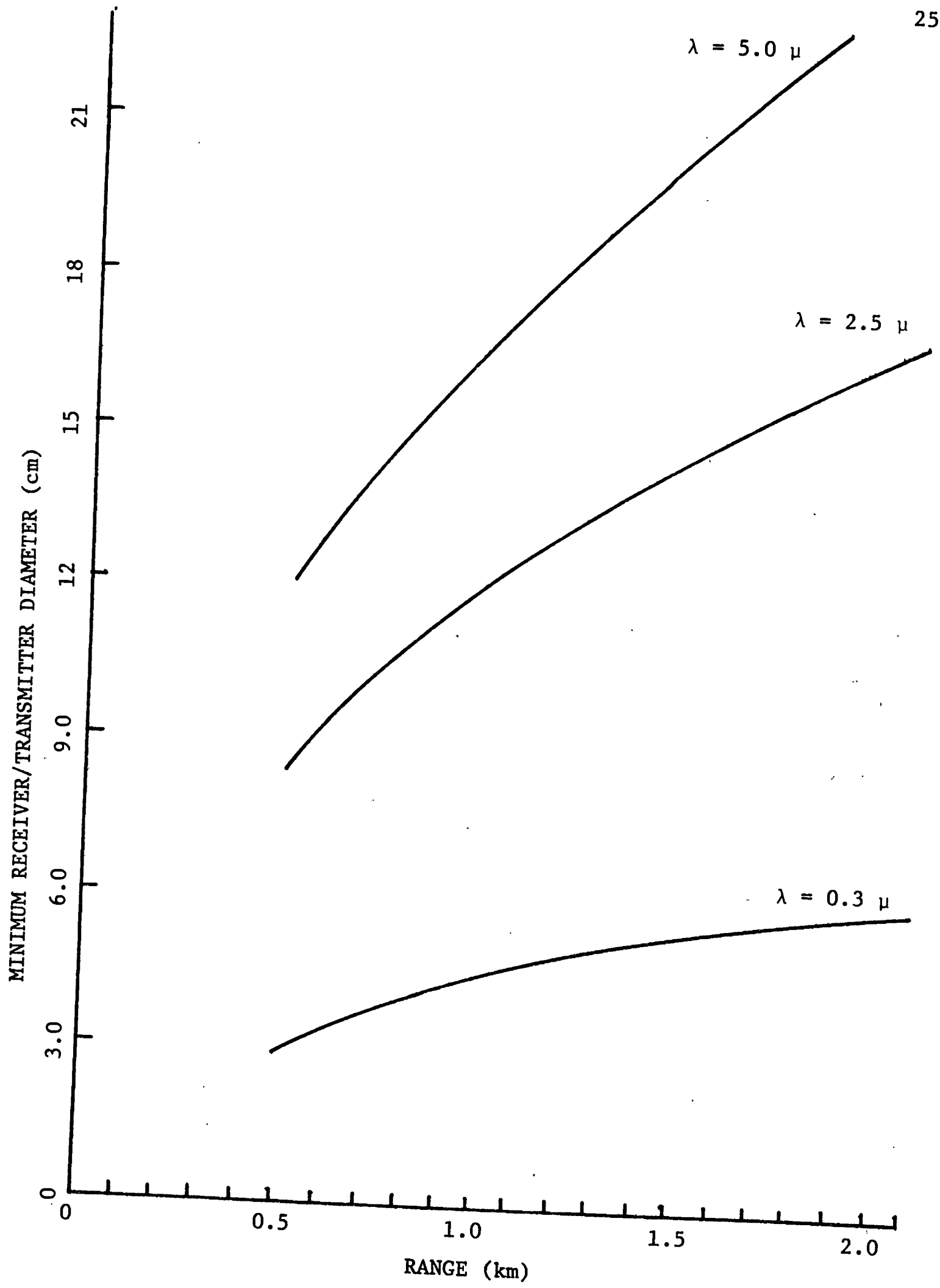
Fig. 5. Minimum diameter laser beam for which 99 percent of the
transmitted power can be propagated between two points. 
quite sma11, being on the order of tens of centimeters. Having determined the laser beam spot size in the absence of turbulence, it is now possible to estimate the beam size in the presence of turbulence.

Perturbation of the Laser Beam by Atmospheric Turbulence. As was stated earlier, the net effect of atmospheric turbulence is to cause the beam to spread out and to wander about the receiver plane. In this section, the magnitude of turbulence caused effects will be estimated. In addition to qualitatively examining the effects of beam steering and beam spreading, beam wander due to diurnal vertical temperature gradients will be considered.

Beam Steering. Consider a plane wave of light which passes through a length of medium whose index of refraction varies due to some perturbation such as a nonuniform temperature profile (see Fig.6). After passing through the medium, various portions of the wavefront will have suffered varying amounts of phase retardation. The phase distortion has the same effect as changing the angle of arrival of the wavefront, that angle being: 23

$$
\Delta \theta=\frac{\Delta \phi}{2 \pi} \cdot \frac{\lambda}{\rho}
$$

where:

$$
\begin{aligned}
\Delta \theta= & \text { the angle of arrival of the perturbed wavefront } \\
& \text { relative to the unperturbed plane wave } \\
\Delta \phi= & \text { the phase retardation between two points along } \\
& \text { the wavefront separated by a distance } \rho .
\end{aligned}
$$

Since the turbulence moves through the beam, the angle of arrival of the wavefront is a quantity whose variation as a. function of time may not be known, but whose statistics can be and have been measured. 


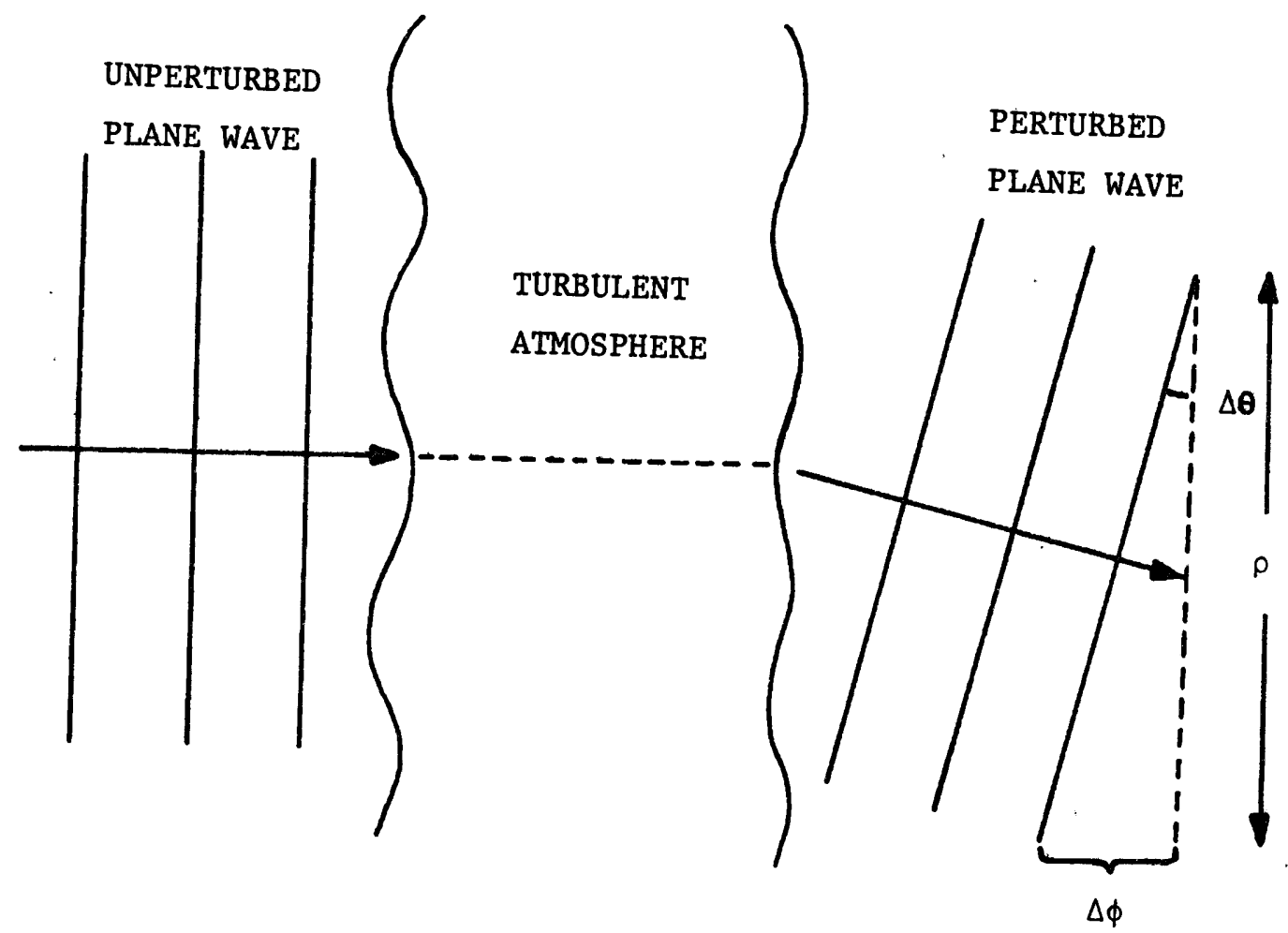

Fig. 6. Schematic representation of the perturbation of a plane
wave on propagating through a turbulent medium. 
The statistical quantities which are used are the standard deviation of the phase fluctuations, $\sigma_{\phi}(\rho)$, and the standard deviation of the angle of arrival fluctuations, $\sigma_{\theta}(\rho)$. The values of the two quantities are taken to be

$$
\sigma_{\phi}(\rho)=\left\langle\Delta \phi^{2}-\langle\Delta \phi\rangle^{2}\right\rangle^{1 / 2}
$$

and,

$$
\sigma_{\theta}(\rho)=\left\langle\Delta \theta^{2}-\langle\Delta \theta\rangle^{2}\right\rangle^{1 / 2}
$$

and are related by:

$$
\sigma_{\theta}(\rho)=\frac{\sigma_{\phi}(\rho)}{2 \pi} \cdot \frac{\lambda}{\rho}
$$

Tatarsl:i 26 postulated that for paths of uniform turbulence intensity, the standard deviation of the phase fluctuations between two points in a beam $\rho$ apart, would have a functional dependence upon the wavelength and pathlength of:

$$
\sigma_{\phi}(\rho)=\frac{3.7 \rho^{5 / 6} C_{n} \mathrm{I}^{1 / 2}}{\lambda}
$$

where $C_{n}$ is an experimentally measured parameter known as the structure constant. Inserting (2.14) into (2.13):

$$
\sigma_{\theta}(\rho)=\frac{1.48 C_{n} L^{1 / 2}}{\rho^{1 / 6}}
$$

The lateral deflection of the beam in the receiver plane will be just:

$$
\delta=\sigma_{\theta}(\rho) L \text { L. }
$$

The actual size of the spot in the receiver plane will be the sum of 
the minimum spot size in the absence of turbulence, plus the amount of lateral deflection due to turbulence.

Taking the minimum spot size to be $3 w(z)$, and assuming it is constant over the path, we get for the size of the beam in the receiver plane, $z=L$ :

$$
\begin{aligned}
D & =3 w(z)+2 \sigma_{\theta}\{3 w(z)\} z \\
& =2.4 \lambda^{1 / 2} L^{1 / 2}+2.56 C_{n} L^{17 / 12} \lambda^{-1 / 12} .
\end{aligned}
$$

This the diameter of the collector necessary to insure that the beam will be entirely captured 39 per cent of the time, and is plotted versus pathlength $\mathrm{L}$ in Figs. $7 a, 7 b$, and $7 c$ for the worst case condition of strong turbulence $\left(C_{n}=5 \times 10^{-7} \mathrm{~m}^{-1 / 3}\right), 2.3$ and for various values of wavelength. The minimum receiver diameter calculated from (2.9) is also shown for comparison.

The time value of 39 percent corresponds to one standard deviation of a Rayleigh distributed variable such as beam deflection. ${ }^{23}$ To increase the fraction of time the beam is completely captured to 99.9 percent, it will be necessary to increase the receiver collector diameter by a factor of three. As can be seen from Figs. 7a, 7b and $7 \mathrm{c}$, even for $1 \mathrm{~km}$ pathlengths receiver diameters on the order of $0.5 \mathrm{~m}$ will be necessary. 'Such collectors are not only expensive, but would require elaborate and expensive mounting fixtures.

Beam Spreading. Beam spreading is the second major turbulence caused effect which will influence the size of the beam in the receiver plane and will be appreciable when the transmitted beam diameter is larger than the turbulence blobs. The mathematical arguments 


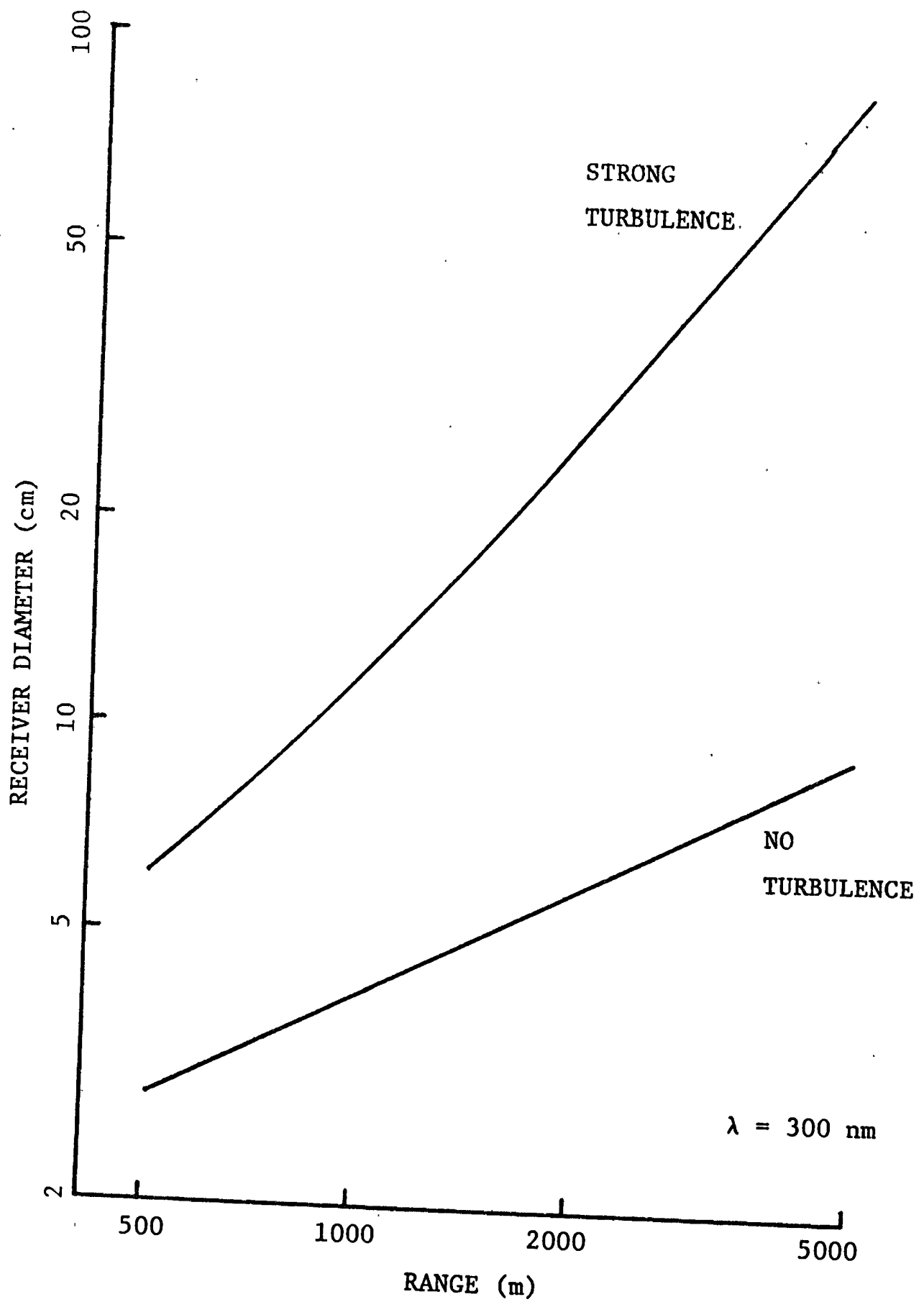

Fig. 7a. Minimum receiver size necessary to capture 99 percent and worst case atmospheric conditions. 


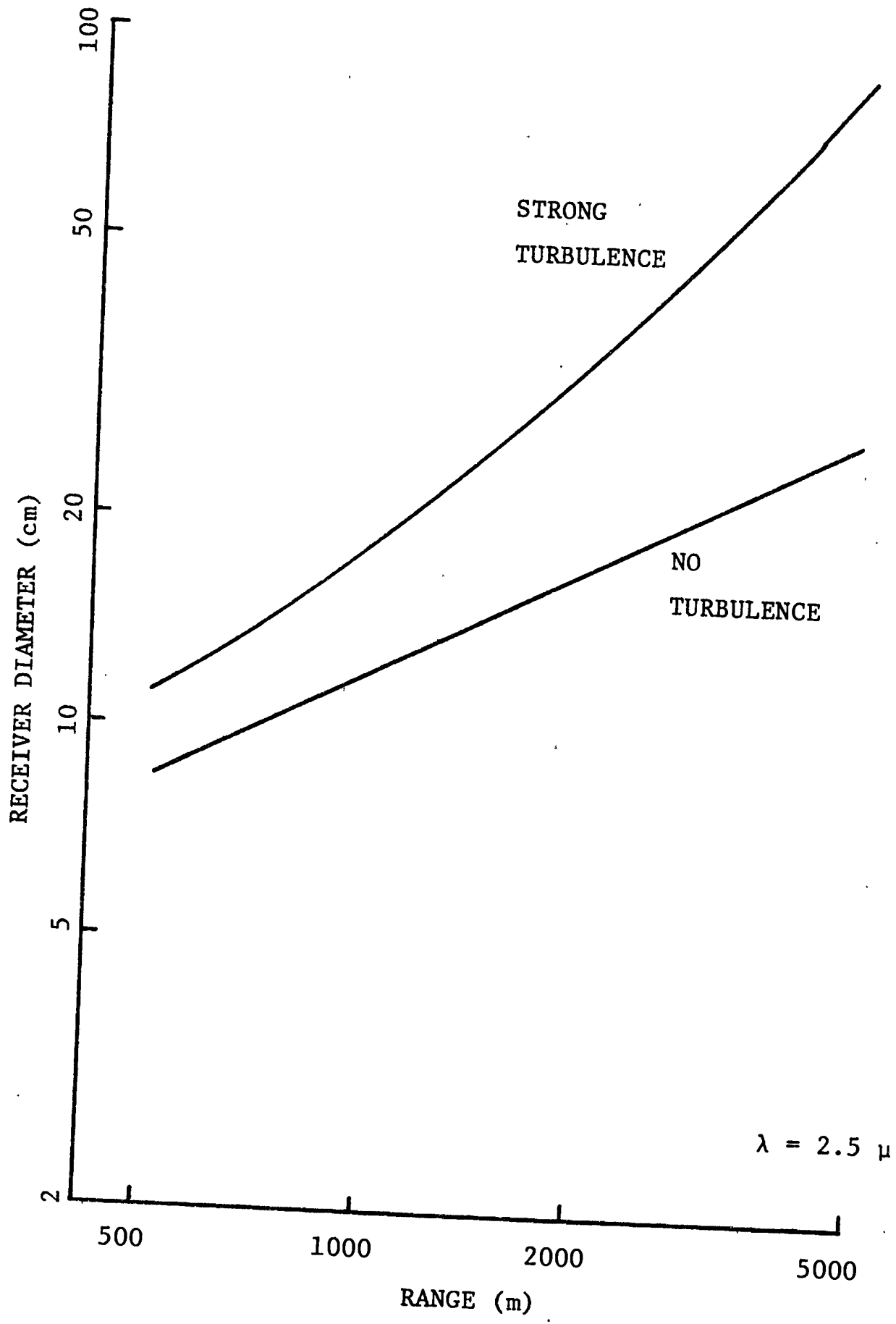

Fig. 7b. Minimum receiver size necessary to capture 99 percent and worst case atmospheric conditions. 


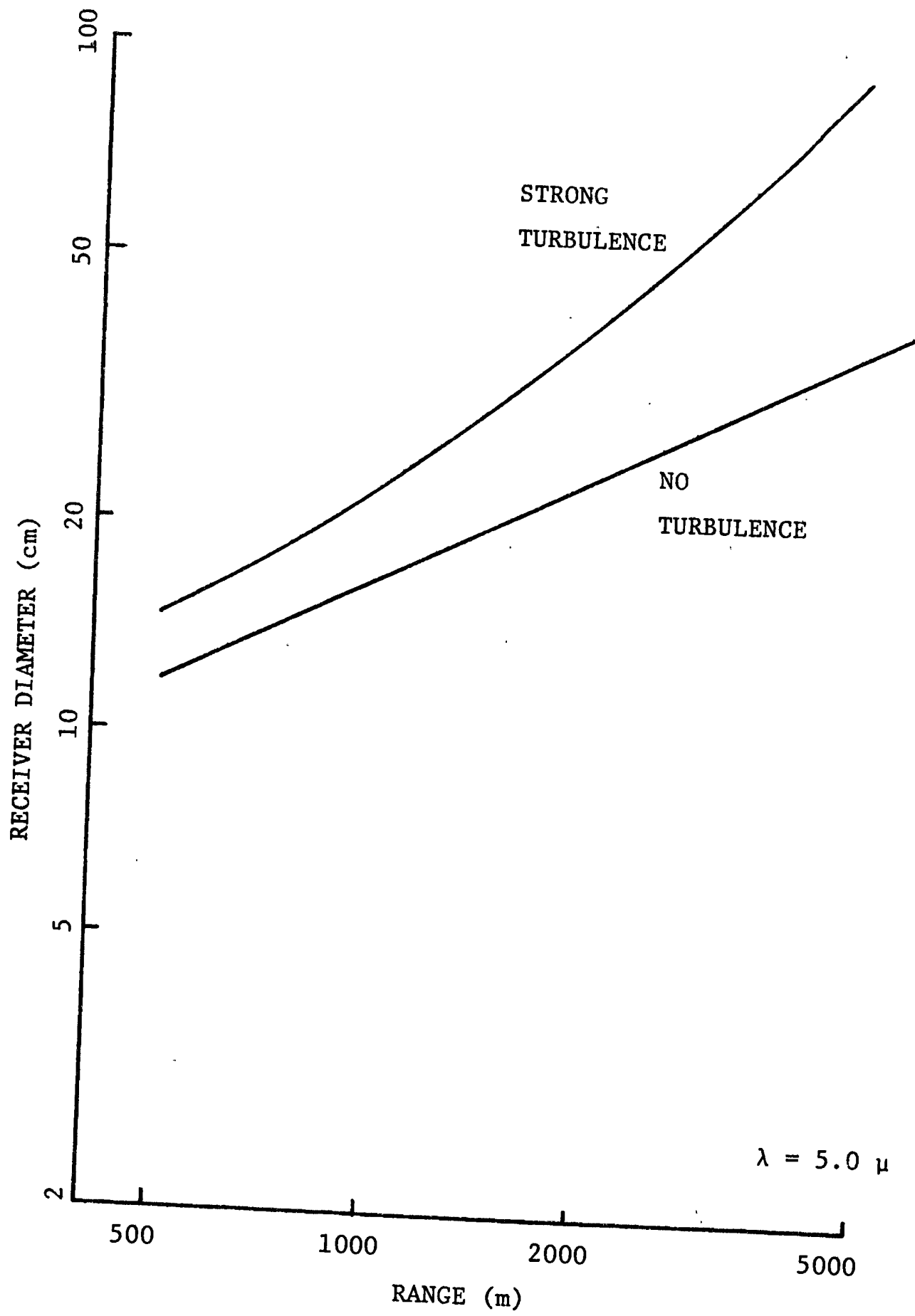

Fig. 7c. Minimum receiver size necessary to capture 99 percent and worst case atmospheric conditions. 36 percent of the time under best 
used to calculate the fraction of the transmitted power collected by a receiver in the presence of beam spreading are far more complicated to understand than those relating to beam steering and only the end result of the mathematical development will be given here. Again following the arguments given by Davis, the fraction of the transmitted power received by a collector of diameter $D$ is given by: ${ }^{23}$

$$
n=\frac{1}{t} \sum_{k=0}^{\infty}\left[\sum_{n=k+1}^{\infty} \frac{t^{n}}{n !} e^{-t}\right]\left[\sum_{n=k+1}^{\infty} \frac{s^{n}}{n !} e^{-s}\right]
$$

where

$$
\begin{aligned}
& t=\frac{0.2 \lambda^{7 / 5}}{L^{11 / 5} C_{n}^{12 / 5}} \\
& s=\frac{.07 D^{2} \lambda^{2 / 5}}{L^{16 / 5} C_{n}^{12 / 5}}
\end{aligned}
$$

and the structure constant $C_{n}$ is taken to have a value of $5 \times 10^{-7} \mathrm{~m}^{-\frac{1}{3}}$, a value corresponding to high turbulence conditions. This expression was numerically evaluated on the Oregon Graduate Center's Prime computer for several wavelengths and pathlengths, and the results of this computation are shown in Fig. 8. As can be seen, large diameter collector optics will be needed to collect the entire transmitted beam under worst case turbulence conditions for large ranges. However, for moderate ranges of $1 \mathrm{~km}$, the collector size is not unreasonable.

Beam Wander Due to Diurnal, Vertical Temperature Gradients. A laser beam propagating over a horizontal surface will experience bending due to the changes in the temperature gradient of the air 


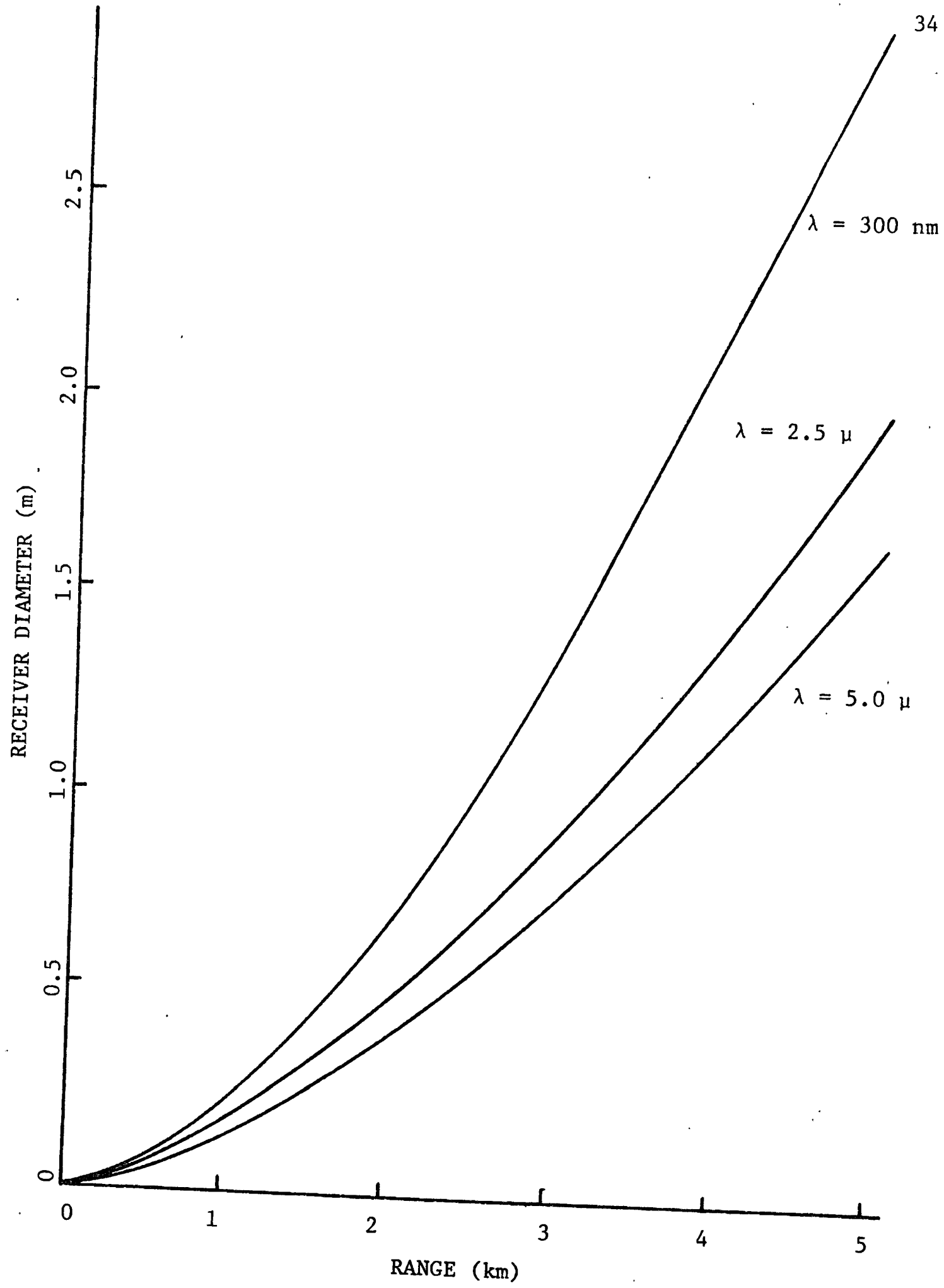

Fig. 8. Receiver diameter necessary to collect 95 percent of the itions, and assumes that ther high turbulence beam-spreading conditions, and assumes that the beam is initially confocally focussed. 
above the ground. During the day when the earth's surface is warmer than the surrounding air, the temperature gradient will be negative. For a normal atmosphere the lapse rate will be on the order of -.006 to $-.01^{\circ} \mathrm{C}$ per meter of vertical rise. ${ }^{22}$ At night, the earth is cooled both convectively and radiatively, and the lapse rate can become positive. While the value of the lapse rate under these conditions is highly variable, a worst case number for night time conditions might be half of the daytime value or +.003 to $+.005^{\circ} \mathrm{C}$ per meter. ${ }^{22}$ The curvature of a horizontal beam near sea level is given by: ${ }^{22}$

$$
C=32.6+930 \frac{\mathrm{dT}}{\mathrm{dh}}
$$

where:

$$
\begin{aligned}
& C= \text { the curvature in microradians per kilometer of } \\
& \text { pathlength }
\end{aligned}
$$

The value of the curvature will be virtually independent of wavelength, since air has little dispersion. In our case we are not interested in the curvature itself, but in the change of curvature which will be from (2.22):

$$
\Delta c=930 \Delta \frac{d T}{d h}
$$

Assuming a worst case change between the day and night lapse rate of $.015^{\circ} \mathrm{C} /$ meter, we get a maximum change in the curvature (which can be thought of as an angular deviation of the beam) of $\Delta C=14$ rradians per $\mathrm{km}$ of pathlength. Compared to turbulence induced fluctuations, this value of beam deviation is small and can probably be neglected. 
Methods for Reduction of Turbulence-Induced Errors. From the preceding discussion on turbulence, it is clear that in general it will not be possible to eliminate the fluctuations in received power without resorting to large collector systems. Large collector systems are undesirable from the standpoint of both size and cost. In fact it would be highly desirable to devise a method other than a large collector to reduce the turbulence-caused fluctuations to near zero. In the next section, two possible methods of reducing turbulence-induced errors will be discussed. The first method takes advantage of the fact that the fluctuations are basically low frequency effects, and that it may be possible to complete a multiple wavelength measurement within a time interval which is short compared to the time required for the turbulence to change. If the turbulence level does not change, then the loss associated with the turbulence will be constant and can be negated using a single dual wavelength measurement. The second method to be examined is that of averaging a large number of pulses to obtain the mean value of the transmitted power at the various wavelengths.

Fluctuation Reduction by Near Simultaneous Sampling. The first method of reducing the fluctuations in the received power is to perform a multiple wavelength measurement in a time short enough to "freeze" the turbulence. The amplitude fluctuations are produced by index of refraction inhomogeneities which are propelled through the beam by the wind, and will therefore have a frequency distribution which is related to the windspeed. Tatarski found experimentally that the frequency component of the amplitude fluctuations $f$, is related to the component of the wind speed perpendicular to the optical path $V$, 
by : 26

$$
f \sim \frac{.3 \mathrm{~V}}{\lambda \mathrm{L}}
$$

and that the high frequency end of the spectrum was roughly $1 \mathrm{kHz}$.

The worst case frequencies will be the highs since they will place a limit on the time interval during which the turbulence can be assumed to be stationary.

Assuming that the power fluctuation varies sinusoidally with time according to:

$$
P=\frac{P}{2}[1+\sin (2 \pi f t)]
$$

then the maximum rate of change of received optical power will be given by:

$$
\frac{d P}{d t}=\pi P_{0} f
$$

where $d p$ is the change in power occurring in a time increment dt; (2.26) can be rewritten as:

$$
d t=\frac{1}{\pi-f} \frac{d P}{P_{0}}
$$

The value of $\mathrm{dt}$. is the maximum amount of time which can lapse within a set of multiple wavelength measurements before the received power fluctuation due to turbulence becomes larger than the fraction $\mathrm{dP} / \mathrm{P}_{\mathrm{o}}$. For example, assuming the maximum allowable change in received power due to turbulence to be $0.05 \mathrm{~F}_{\mathrm{o}}$, and a maximum value for $\mathrm{f}$ of $1 \mathrm{kHz}, 26$ then the maximum time between samples for which the turbulence will be frozen is 16 microseconds. It will be difficult to mechanically move 
any tuning elements within a laser such as a dye laser within that time span; however, methods of tuning a laser exist which do not necessitate physically moving an optical element, and will be discussed in chapter VI.

Fluctuation Reduction by Pulse Averaging. Since the turbulence-induced fluctuations in received power are random, a second method which suggests itself to reduce the errors caused by the fluctuations is to average a large number of pulses to obtain the mean value of the transmitted power. It can be argued that the mean value of the received power will provide the concentration information since the average power lost due to turbulence could just as easily be attributed to some loss mechanism such as scattering.

If pulse averaging is to be used to reduce the fluctuations in the received power, the question arises as to how many pulses must be sampled to obtain a mean value which statistically is equal to the actual mean value without fluctuations within some precision or confidence limit. Confidence limits have been worked out for the case of normal or Gaussian probability distributions, but not for Rayleigh distributions, which describe the fluctuation in received power. ${ }^{23}$ It can be shown however that the probability distribution for the sum of a number of non-normal distributions will approximate a normal distribution provided that the non-normal distribution samples are independent. This is known as the central 1imit theorem. ${ }^{28}$ For multiple samples of the received power to be statistically independent, the samples or pulses must be decorrelated, and therefore separated in time by the decorrelation time. Decorrelation times for light passing through the 
atmosphere are on the order of $10 \mathrm{~ms}$ or 1 ess, ${ }^{29}$ and therefore the pulse rate of the laser is limited to $100 \mathrm{~Hz}$. For cases of weak turbulence the decorrelation time is longer, but under those conditions it should be possible to collect the entire beam negating the need for pulse averaging. Assuming that the received pulses are decorrelated, then the mean square relative error in the received power $\varepsilon$ is: ${ }^{30}$

$$
\varepsilon=\frac{\sqrt{2} \sigma}{\mathrm{P}} \frac{1}{\sqrt{n}}
$$

where:

$$
\begin{aligned}
& \sigma=\text { the standard deviation of the received power } \\
& \overline{\mathbf{p}}=\text { the mean value of the received power } \\
& \mathbf{n}=\text { the number of uncorrelated samples. }
\end{aligned}
$$

While it is not possible to assign particular values to the standard deviation of the received power without knowledge of the atmospheric conditions present at the time of the pollutant measurement, a worst case value is $\sigma=\frac{\bar{p}}{3}$. Making the further assumption that the error in the mean value of the received power should not exceed 0.5 percent, one finds that approximately 8,900 pulses would have to be averaged. If the pollutant monitor were operating at the maximum pulse rate of $100 \mathrm{~Hz}$, then 178 seconds would be required to complete a single dual wavelength measurement. While this number of pulses is larger than might be desired, it is not prohibitively large. Moreover, the necessary number of pulses can be rapidly reduced by increasing the size of the receiver collector over the minimum size required in the absence of turbulence. The reason is quite simple. As the level of the turbulence increases and the beam begins to spread out, various portions of the beam will pass through spatially different and uncorrelated turbulence patterns. The result of the beam passing 
through uncorrelated turbulence paths is that the beam in the receiver plane will consist of patches of light which are themselves uncorrelated. Each of the uncorrelated patches can in turn be thought of as a separate pulse. A rigorous calculation is not required to appreciate the importance of this result, since the largest diameter patch of correlated light which can exist in the receiver plane is one which has not been perturbed by the atmosphere. For example, the use of a receiver which is three times the minimum size as given by (2.9) would intercept at least seven uncorrelated patches of light and thus allow a sevenfold reduction in the number of pulses required. In fact the reduction in the number of pulses required is even greater, since as the level of turbulence increases, the size of the correlated patches of light decreases. ${ }^{23}$

Thus at least two methods of reducing the effects of turbulence appear possible, and which one is adopted will depend upon the operating characteristics of the laser light source selected. 


\section{CHARACTERIZATION OF SPECTRAL REGIONS}

In this chapter the optical spectrum will be broken up into a number of spectral regions. 33 Each spectral region will be examined in terms of the existence of pollutant absorption spectra and the strength of the absorption. It will be particularly important to identify those spectral regions which display absorption spectra of pollutants for which federal emission limits have been established. Those pollutants and their concentration limits are shown in Table 1.32

Vacuum U1traviolet: $10-200 \mathrm{~nm}$

The term vacuum ultraviolet is derived from the practice of evacuating laboratory spectrometers used in this spectral region, since vacuum ultraviolet light is quickly absorbed by molecular oxygen. 33 Because of the strong absorption by oxygen, this spectral region is not useful for making long-path measurements.

\section{Near U1traviolet: $200-380 \mathrm{~nm}$}

Absorption spectra in this spectral region originate from electronic transitions ${ }^{33}$ in atoms and molecules and are typically very strong. While not all pollutant gases display absorption spectra in this region, those pollutant molecules which possess permanent dipole moments do. 34 For example, sulfur dioxide is an important pollutant 
TABLE I

FEDERAL CONCENTRATION STANDARDS 32

FOR FIVE IMPORTANT POLLUTANTS

\begin{tabular}{|c|c|c|c|}
\hline Pollutant & $\begin{array}{l}\text { Averaging } \\
\text { Time }\end{array}$ & $\begin{array}{l}\text { Primary } \\
\text { Standard }\end{array}$ & $\begin{array}{l}\text { Secondary } \\
\text { Standard }\end{array}$ \\
\hline $\begin{array}{l}\text { Photochemica } 1 \\
\text { Oxidants. }\end{array}$ & $1 \mathrm{hr}$ & $0.08 \mathrm{ppm}$ & $0.08 \mathrm{ppm}$ \\
\hline \multirow[t]{2}{*}{ Carbon Monoxide } & $8 \mathrm{hr}$ & $9.0 \mathrm{ppm}$ & $9.0 \mathrm{ppm}$ \\
\hline & $1 \mathrm{hr}$ & $35.0 \mathrm{ppm}$ & \\
\hline \multirow{2}{*}{$\begin{array}{l}\text { Nitrogen } \\
\text { Dioxtde. }\end{array}$} & Annual Average & $0.05 \mathrm{ppm}$ & $0.05 \mathrm{ppm}$ \\
\hline & $1 \mathrm{hr}$ & $0.25 \mathrm{ppm}$ & \\
\hline \multirow{4}{*}{$\begin{array}{l}\text { Sulfur } \\
\text { Dioxide }\end{array}$} & Annual Average & $0.03 \mathrm{ppm}$ & \\
\hline & $24 \mathrm{hr}$ & $0.14 \mathrm{ppm}$ & \\
\hline & $3 \mathrm{hr}$ & & $0.5 \mathrm{ppm}$ \\
\hline & $1 \mathrm{hr}$ & $0.5 \mathrm{ppm}$ & \\
\hline Hydrocarbons & $\begin{array}{l}3 \mathrm{hr} \\
(6-9 \text { A.M. })\end{array}$ & $0.24 \mathrm{ppm}$ & $0.24 \mathrm{ppm}$ \\
\hline
\end{tabular}


primarily associated with fossil fuel fired electrical power generating plants, ${ }^{35}$ and displays a rapidly varying absorption spectra from rough1y 280 to $310 \mathrm{~nm} .{ }^{36}$ Like $\mathrm{SO}_{2}$, nitrogen dioxide also has a rapidly varying absorption spectra with wavelength, with absorption from $\sim 300 \mathrm{~nm}$ to beyond the long wavelength end of the near ultraviolet. ${ }^{37}$ Ozone displays intense absorption below $30 n \mathrm{~nm},{ }^{39}$ but unfortunately its spectrum is that of a smooth continuum, making multiple wavelength measurements unfeasible.

Visible: $\quad 380-780 \mathrm{~nm}$

The visible region of the electromagnetic spectrum is a region where very few gases absorb 1ight. In fact the only major pollutant exhibiting reasonably strong absorption in this spectral region is nitrogen dioxide, and it has been measured over long paths using a two wavelength argon ion laser. ${ }^{39}$ Because of the virtual lack of absorption spectra in the visible, this spectral region has not been a serious contender for use in pollutant monitoring.

Near Infrared: $.78-3.0 \mu$

In general, most molecular gases exhibit absorption throughout the infrared due to molecular vibrational-rotational transitions. In the near infrared, harmonics of molecular vibrational-rotational transitions appear. These harmonics are several orders of magnitude less intense than the fundamental transitions and are generally too weak to be useful in pollutant detection. ${ }^{16}$

\section{Middle Infrared: $3-30 \mu$}

The middle infrared spectral region is known as the "fingerprint" 
region, for in it most molecules have absorption spectra which differ in shape, strength and spectral location. ${ }^{16}$ The absorption spectra, while not as strong as those found in the ultraviolet, are in general strong enough to enable one to make sensitive concentration measurements of a number of gases. The middle infrared has an advantage over the ultraviolet in that a number of pollutants which do not exhibit usable absorption spectra in the ultraviolet, such as $C O$, possess such spectra in the infrared.

As in the ultraviolet, portions of the middle infrared are unusable due to spectral interference from certain gases. The interferences are due primarily to absorption from carbon dioxide and water vapor. In particular carbon dioxide absorbs from approximately 4.2. microns to 4.5 microns, while water vapor has absorption bands between 2.5 and 3.3 microns, and 4.9 to 7.2 microns, ${ }^{17}$ all of which are extremely strong, making it unlikely that a laser beam within these wavelength regions could be propagated over any useful range. However, a number of important pollutants such as carbon monoxide, 40 ozone, 41 and sulfur dioxide ${ }^{41}$ have absorption spectra which lie within spectral regions not affected by blanket absorption.

Far Infrared: $\quad 30-300 \mu$

A number of molecules possess vibrational-rotational spectra in the far infrared. The region is rendered unusable for absorption measurements by very intense continuum water vapor absorption. 16

Microwave: $300-1,000,000 \mu$

Molecular rotational lines appear in the microwave region; 
however, the absorption lines in the region suffer from extreme pressure broadening. 16 This broadening causes the absorption lines to overlap to such an extent that they are unusable for making long-path measurements.

\section{Summary of Spectral Regions}

From the preceding discussions it is clear that of the six spectral regions cited, only the ultraviolet and middle infrared regions have absorption spectra which are generally suitable for making long-path measurements. It should be noted that problems exist in both regions which will cause difficulty in making such measurements. For instance, both regions suffer interference from scattering. Interference due to spectral overlap may be a problem in the infrared, and will almost certainly be encountered in the ultraviolet. Which of the spectral regions is selected will depend upon the availability of tunable laser light sources within that spectral region, a topic to be covered in the next chapter. 


\section{CHAPTER IV}

\section{EXAMINATION OF AVAILABLE TUNABLE LASER SOURCES}

As was noted in the introduction, it is highly desirable to use a laser light source when making long-path absorption measurements. The characteristics of a laser which make them ideally suited for use as a light source are:

1. highly collimated light beam

2. intense beam

3. highly monochromatic light and does not require dispersive elements in the receiver.

Perhaps the primary problem which has limited the application of lasers to long-path monitoring is the need to match a laser's emission wavelength to the wavelength of a pollutant's absorption line. Despite the large number of pollutant absorption lines and laser wavelengths avai1able for such matches, only a few coincidences have been found. In fact, for the important pollutant sulfur dioxide, no useful coincidence had been shown to exist at the initiation of this study. What is desired then is a laser device which could be tuned to match a particular pollutant's absorption line. The additional need to use multiple wavelengths as a means of discriminating against the various forms of interference makes it almost imperative that some type of tunable source that can emit 1 ight both on and off resonance with the absorbing gas be used. Fortunately, a few such tunable laser devices do exist, 
both for use in the infrared and u1traviolet. In the infrared the relevant devices or processes are:

1. High pressure gas lasers

2. Junction type semiconductor lasers

3. Spin-flip raman lasers

4. Parametric oscillator

5. Difference frequency generation

In the ultraviolet three devices/techniques are available for the production of tunable light. They are:

1. Dye lasers

2. Excimer lasers

3. Frequency doubling of a tunable visible dye laser

In the next section, these various tunable sources will be discussed in terms of their applicability to pollutant monitoring.

\section{Infrared Devices}

High Pressure Gas Lasers. Continuously tunable molecular gas lasers $^{42}$ rely upon the ability to pressure broaden the closely spaced vibrational gain lines of the laser medium to the extent that they overlap. As an example, consider pressure broadening the emission lines of the carbon dioxide 1aser. $\mathrm{CO}_{2}$ has a large number of laser lines spaced rough1y $20 \mathrm{~nm}$ apart and centered around $10.6 \mu{ }^{43}$

By raising the pressure of the gas in the $\mathrm{CO}_{2}$ laser to roughly

10 atmospheres, 44 it is possible to pressure broaden the $\mathrm{CO}_{2}$ emission lines to such an extent that the laser can be continuously tuned over the entire emission spectrum which extends roughly from 9 to $11 \mu .43$ Unfortunately this pressure is many times higher than typical 
operating pressures found in continuous and pulsed $\mathrm{CO}_{2}$ lasers. Laser operation at pressures greater than a few atmospheres can be achieved but not without considerable difficulty because of problems in obtaining the uniform electrical discharge necessary for laser pumping. In fact the widespread future use of high pressure gas lasers is predicated upon the development of simple, efficient, and inexpensive methods of excitation. Recent progress in the area of waveguide 1 asers 95 looks promising; however, an acceptable method of excitation has yet to be fully developed.

Junction Type Semiconductor Lasers. Junction type semiconductor lasers, ${ }^{46}$ or diode lasers as they are normally termed, emit light when an exited atom in the junction region of a semiconductor changes from the excited donor state to the unexcited, or acceptor leve1. The energy and therefore the wavelength of the emitted photon is dependent upon the width of the bandgap and, since the width of the bandgap is largely dependent upon the composition of the diode, it is possible in theory to produce diode lasers which operate at virtually any wavelength by varying the composition of the semiconductor materials. In fact, lasing has been observed from such devices over a wavelength region extending from 630 nanometers to 31 microns. 46 The light produced by these devices is highly divergent due to the small cross sectional area of the active region and must be collimated by a lens. Since the junction region is physically much wider than it is high, the emergent light has two distinctly different angles of divergence. Thus collimation cannot be satisfactorily achieved with normal spherical optics. In addition many of these laser devices require refrigeration 
to liquid nitrogen temperatures and below. Another problem associated with laser diodes is that unlike most other tunable laser sources whose output wavelength is primarily dependent upon some external tuning element such as a prism the output wavelength of diode lasers is critically dependent upon conditions existing in the junction. While some of the junction conditions, such as temperature, pressure, and magnetic field can be assets in that they can be used as tuning mechanisms, others such as changing chemical composition due to aging, are beyond the control of the user. Achieving long term wavelength stability is a problem. However, none of the aforementioned problems are insurmountable, as evidenced by the recent availability of a laboratory spectrometer ${ }^{48}$ using a tunable diode laser as a light source. The present cost of a diode laser capable of operating in the middle infrared is several thousand dollars. ${ }^{49}$ This cost could possibly be reduced to ten to one hundred dollars if mass produced; ten to one hundred dollars is the present cost of gallium arsenide diode lasers which operate at .9 microns. ${ }^{49}$ Such a low cost would help offset the cost of the wavelength control equipment. At the conception of this project, laser diodes in the middle infrared were not commercially available.

Parametric Oscillators. A parametric oscillator ${ }^{50}$ is a device in which a pump photon at a wavelength $\lambda_{p}$ is split into a signal photon at $\lambda_{s}$ and an idler photon at $\lambda_{i}\left(\lambda_{p}<\lambda_{s}<\lambda_{\dot{I}}\right)$. The splitting is subject to the conservation of energy relation:

$$
1 / \lambda_{p}=1 / \lambda_{s}+1 / \lambda_{i}
$$


and is accomplished in a nonlinear optical medium such as a noncentrosymetric crystal. A large number of materials have been examined as possible candidates for parametric oscillators, and in fact one crystal, lithium niobate, was used in a commercially available instrument. 51 For the parametric oscillator process of splitting pump photons into longer wavelength signal and idler photons to be efficient, it is necessary to focus the pump beam to intensities which approach the damage threshold of the crystal. The necessity of operating the parametric oscillator at pump powers which are near the damage threshold of the crystal, places stringent requirements upon the repeatability of the peak power output from the pump laser. Such requirements almost certainly increase the cost of an already expensive pump laser. The cost of a parametric oscillator in 1973 was around $\$ 40,000,,^{51}$ of which most of the expense was in the Nd:YAG pump laser. This high cost probably removes the IR parametric oscillator from consideration as a light source for routine, long-path pollutant monitoring. It should, however, be noted that Byers has a laboratory parametric oscillator which has been used to monitor pollutants in the San Francisco Bay area. The developmental cost of this laboratory prototype system was on the order of $\$ 400,000.52$

Spin-Flip Raman Laser. A spin-flip raman laser ${ }^{53}$ is a laser device which generates tunable infrared light by frequency shifting the output from a non-tunable pump laser. The principle of operation is quite simple, being very similar to conventional spontaneous raman scattering. In spontaneous raman scattering, light which is incident upon a raman material such as hydrogen gas is frequency shifted by 
exciting a vibrational mode within the molecule. Excitation of the vibration of frequency $\nu_{v}$ by an incident pump photon of frequency $\nu_{p}$, reduces the energy of the pump photon by an amount $h v_{v}$. The resultant frequency shifted photon is called the Stokes photon and has a frequency $v_{s}$ given by: 54

$$
v_{s}=v_{p}-v_{v}
$$

In a spin-flip raman laser the pump photons are scattered off electrons in the raman material in such a way as to cause the magnetic dipole associated with the electrons to flip direction; the dipoles are initially aligned by an external magnetic field. The energy required to flip the dipoles comes from the incident pump photon. The amount of energy required can be varied by changing the magnitude of the applied magnetic field. The output frequency of the spin-flip raman laser is given by: 59

$$
v_{s}=v_{p}-\frac{1}{2 \pi} g \mu_{B} B
$$

where:

$$
\begin{aligned}
& g=\text { the electron } g \text { factor } \\
& \mu_{B}=\text { the bohr magneton } \\
& B=\text { the value of the applied magnetic field. }
\end{aligned}
$$

Using a crystal of indium antimonide as the raman material, it has been possible to produce tunable radiation from 11.7 to 13 microns using a carbon dioxide laser pump, 53 and from 5 to 6 microns using a carbon monoxide laser pump. 53 While the theory of operation of a spin-flip raman laser is simple, the laser itself is quite complicated, 54 and 
will probahly not be suitable for use in a long-path monitoring in'strument.

Summary of Tunable Infrared Laser Devices. While a number of tunable laser light sources exist for the middle infrared, all were considered either too expensive, or as in the case of diode lasers, not readily or commercially avallable in the proper spectral regions at the inception of this project. of the light sources discussed, only diode lasers hold the possibility of being Inexpensively produced (lèss than $\$ 1000)$.

\section{Tunable U1traviolet Laser Light Sources}

U1traviolet Dye Lasers. The dye 1 aser 55 is a laser which uses an active medium comprised of an organic dye dissolved in an organic solvent such as methanol. The dye molecules are typically optically excited using either a flashlamp or another laser. Flashlamp pumped dye lasers have the possibility of being inexpensive to construct and operate; however their direct use in the ultraviolet is precluded by the unavailability of organic dyes which lase in the ultraviolet. The only dye commercially produced to date which lases in the uv is p-terpheny 1,56 which emits between 340 and $360 \mathrm{~nm}$. Thus pending the development of new dyes for operation in the middle ultraviolet, the direct use of a dye laser monitoring instrument is precluded.

Excimer Lasers. Laser action in the ultraviolet region of the spectrum from unstable molecules such as $\mathrm{ArF}, \mathrm{KrF}$, and $\mathrm{XeF}$, was first observed in 1975.57 While the possibility exists for tuning the outputs of these lasers by perhaps $\pm 2.5 \mathrm{~nm}$ around the laser emission 
line centers, ${ }^{58}$ the necessity of maintaining a laser containing free fiuorine gas probably precludes the use of these lasers from serious consideration for use in a pollutant monitor.

\section{Frequency-Doubled Tunable Visible Light Sources. Frequency} doubling ${ }^{59}$ refers to a nonlinear optical process much like parametric oscillation. In frequency doubling, however, two pump photons are combined in a nonlinear medium to form a single photon having twice the energy and therefore half the wavelength of the pump photons. The use of recently developed crystals ${ }^{60,61}$ has extended the range of frequency doubling such that it is now possible to produce tunable light at any wavelength from roughly 200 nanometers out to the visible. Moreover, a wide variety of dyes spanning the visible region of the spectrum as well as a variety of pump sources for the dyes exist. For example, to measure $\mathrm{SO}_{2}$ which absorbs in the 280 to $310 \mathrm{~nm} \mathrm{spec-}$ tral region, ${ }^{36}$ one desires a visible source in the 560 to $620 \mathrm{~nm}$ region which can be frequency doubled. This region is covered by the organic dye rhodamine $6 G, 62$ one of the most efficient and widely used laser dyes available. It has been pumped by frequency-doubled Nd:YAG lasers, ${ }^{63}$ nitrogen lasers, ${ }^{64}$ pulsed and continuous ion lasers, 65,66 as well as flashlamps. ${ }^{67}$ Nd:YAG pumping can be ruled out on the basis of price, since the cost of a frequency-doubled Nd:YAG laser can easily exceed $\$ 20,000 .^{49} \mathrm{CW}$ dye lasers must also be ruled out on the basis of' cost since the ion pump lasers alone have typical price tags in excess of $\$ 10,000.49$ Besides, frequency doubling a cw dye laser is difficult due to the lack of high optical power.

\section{Nitrogen Laser Pumped Dye Lasers. Nitrogen lasers typically}


produce pulses of $1 \mathrm{~mJ}$ with a pulse length of $10^{-8} \mathrm{~S} .68$ This corresponds to a peak power of $10^{5} \mathrm{~W}$. Perhaps 10 percent of this power can be converted to dye light in the 580 to $600 \mathrm{~nm}$ region corresponding to a peak dye power of $10^{4} \mathrm{~W} .68$ of this $10^{4} \mathrm{~W}$ of dye light, between 1 and 10 percent $^{78}$ can be converted to second harmonic, thus giving a peak output of 100 to $1000 \mathrm{~W}$ of frequency-doubled dye 1ight. ${ }^{68}$ This amount of ultraviolet could be easily detected using a variety of photodetectots.

A major problem with nitrogen laser pumped dye lasers is that because of the high peak power of the pump pulse, the dye laser will haye an extremely high gain: While this might appear to be an advantage, it can easily become a problem since the laser will have a strong tendency to operate in the superradiant mode, 69 ignoring attempts to control both its spatial mode and spectral width. One could realistically expect to have difficulty getting the dye 1 aser to operate at wavelengths other than those centered about the dye's fluorescent maximum. What would be desirable would be either two nitrogen lasers, one with a low energy output, and a second one with a large energy output, or a single, high-powered nitrogen laser equipped with a beam splitter to split the output into two beams of unequal intensity. 70 The lower-powered of the two beams could be used to pump a small low-gain, dye oscillator. Because of the low gain of this dye laser, it would be possible to maintain tight control over both its spatial mode and spectral bandwidth. The output from this 1ow-powered, but spectrally and spatially well-behaved dye laser could then be passed through a dye amplifier pumped by the second high-powered 
nitrogen laser beam. While this is the desirable method, it adds undesirable complexity to the system. Nonetheless, nitrogen pumped dye lasers were considered as a possible light source.

Excimer Laser Pumped Dye Lasers. Excimer lasers have recently been utilized to pump dyes, ${ }^{71}$ but they were not available at the inception of this study. Even if they had been, short laser lifetimes associated with using free, very highly reactive fluorine or other rare gas-halogen mixtures would be a major disadvantage which would probably preclude their use.

Flashlamp-Pumped Dye Lasers. Organic dye lasers can also be pumped using a high intensity, short pulse flashlamp. ${ }^{67}$ A typical flashlamp-pumped system might use a xenon filled flashlamp with an electrica1 input energy of from a few joules to several hundred joules. Typical electrical input energy to dye laser output energy efficiencies are on the order of 0.1 percent. ${ }^{72}$ If we consider such a system with an input of $20 \mathrm{~J}$ and a pulse length of $1 \mu \mathrm{S}$, one might expect a dye laser output of $2 \mathrm{~kW}$ peak. A 1.0 percent doubling efficiency would result in a UV output of $20 \mathrm{w}$ peak, an amount which is significantly larger than the minimum power necessary for making a long-path measurement. One of the primary problems experienced with a flashlamp-pumped system is that of thermo-optic distortion in the dye medium due to the large amount of energy intercepted by the dye from the flashlamp. ${ }^{73}$ The effect of introducing large amounts of energy into the dye solution is nonuniform heating of dye which causes large index of refraction variations across the dye cell; these in turn cause the dye laser beam to break up spatially. Such a beam will be 
difficult to focus to the high intensities necessary for efficient frequency doubling. Fortunately this problem can be partially alleviated by using solvents such as water which have a smaller change in refractive index with temperature, and therefore less of a tendency to cause beam breakup than the customarily used solvents such as methanol.

\section{Choice of Optimum UV Laser}

Two dye laser pumping systems are available which could produce useful amounts of second harmonic while requiring only relatively low costs. Since the cost of construction is a highly relevant parameter in the selection of any dye laser for use in a pollution monitoring system, the nitrogen and flashlamp-pumped dye lasers were examined in terms of their material cost. The estimated material cost of building the various frequency-doubled dye laser systems is shown in Table II. As can be seen from Table II, both of the proposed systems appeared to have roughly the same component costs. The main cost in either system will be labor, a cost which could easily amount to $\$ 5,000$ per laser. It should also be pointed out that the aforementioned estimates are the costs of the light source alone, and do not reflect the additional cost of the detector and data handing electronics. This cost could easily amount to an additional $\$ 10,000$, thus raising the tota1 system cost to around $\$ 20,000$.

While both the nitrogen laser pumped dye laser and the flashlamppumped dye laser appeared to have the same component costs, the nitrogen laser pumped system was thought to have a number of disadvantages compared to a flashlamp-pumped dye laser. The high voltage pulse 
TABLE II

PROJECTED COMPONENT COSTS OF CONSTRUCTING A FREQUENCY-DOUBLED DYE LASER

Nitrogen Laser Pumped:

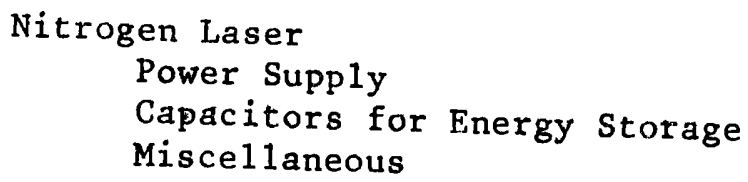
$\$ 2,000$ 250 500

Dye Laser

Dye Ce11

Optics

Dye, Solvent and Pump

Frequency Doubler

Doubling Crystal

Optics

Total

$\$ 3,750$

Flashlamp-Pumped:

Dye Laser

Power Supply

Capacitor

Optics

Dye, Solvent and Pump

Miscellaneous

Flashlamps

$\$ 2,000$

200

350

150

500

200

Frequency Doubler

Doubling Crysta1

Optics 
circuitry necessary for the operation of the nitrogen laser was felt to be significantly more difficult to construct than the electrical driver for the flashlamp-pumped laser. The nitrogen laser pumped dye laser would be more difficult to control, both spatially and spectral1y, than its lower gain, flashlamp-driven counterpart. The flashlamppumped system on the other hand, appeared relatively straight forward to construct. The only problem foreseen, was that of thermo-optic distortion of the dye medium by the flashlamp, a problem which was believed could be overcome through the proper choice of dye solvent and adequate coolant. For this reason it was decided to attempt the construction of a frequency-doubled, flashlamp-pumped dye laser, for use in monitoring sulfur dioxide gas in the near ultraviolet. 
CHAPTER V

OVERVIEW OF PROPOSED LONG-PATH MONITOR

The long-path sulfur dioxide monitoring instrument which was envişioned is shown in Figure 9, and was to use a microprocessor-controlled frequency-doubled dye laser as a source of ultraviolet light in the $300 \mathrm{~nm}$ region. The operation of the complete system would be as follows. The microprocessor would tune the dye laser and frequency-doubler to a wavelength $\lambda_{1}$ corresponding to a minimum in the sulfur dioxide absorption spectrum. The position of the dye laser and frequency-doubler tuning elements which corresponded to this particular wavelength would be programmed into the microprocessor's memory. Once the tuning was completed, the microprocessor would give the command causing the laser to fire. A small fraction of the ultraviolet light would be split from the out-going laser beam and sent to a reference detector. The remainder of the beam would proceed to the telescope where the beam would be expanded to a diameter determined by the arguments in chapter II. At the end of the long path, the beam could be collected and detected, or as is shown in Figure 9, retro-reflected back to the transmitting telescope where it would be separated by a beam splitter from the out-going beam and routed to the return pulse detector. If pulse averaging techniques were adopted to reduce the errors due to atmospheric turbulence, the laser would be repeatedly pulsed until the necessary number of pulses had been collected. The dye laser and frequency doubler would then be tuned to a second 
TRANSMITTING

RETROREFLECTOR

TELESCOPE

TO

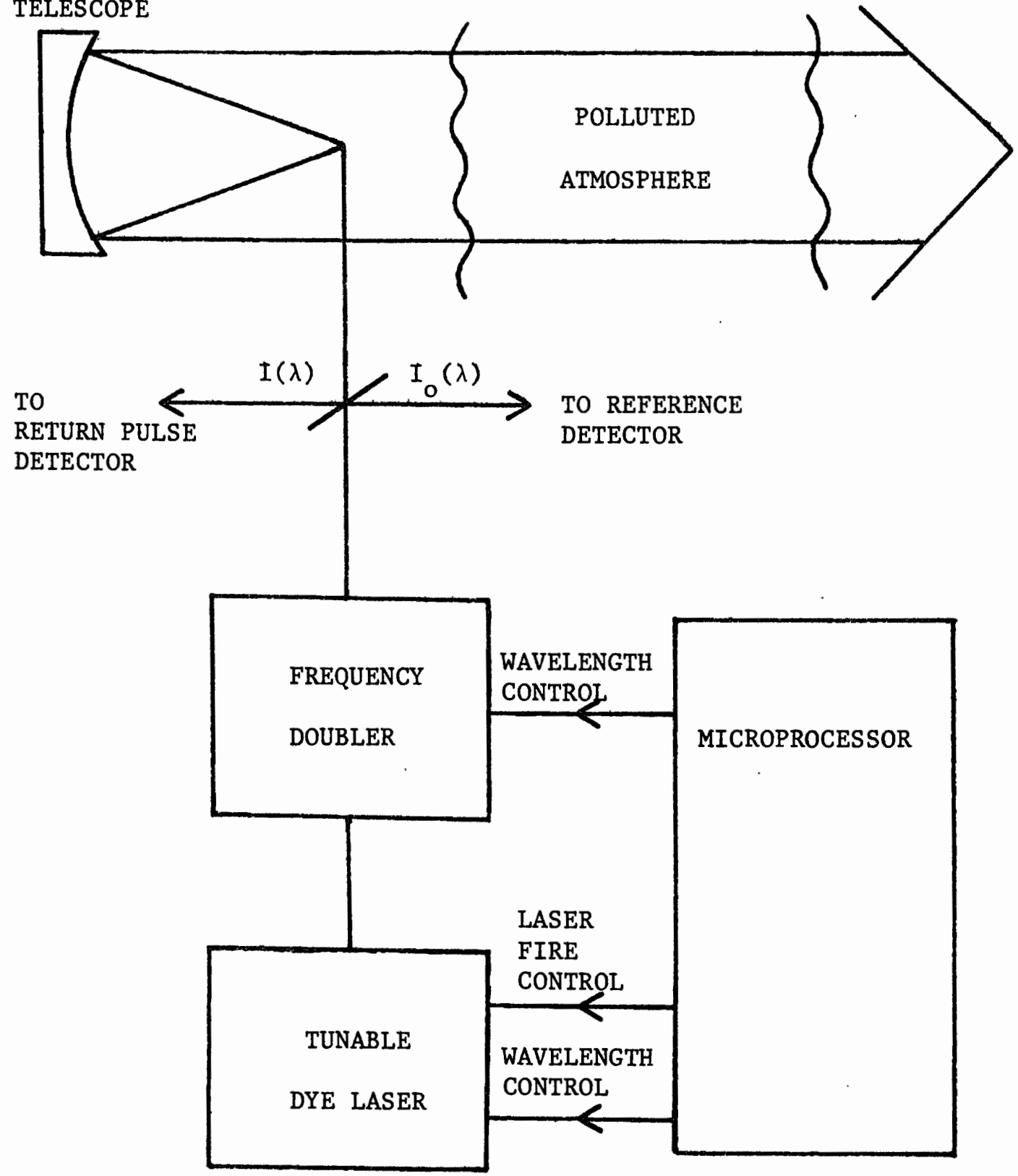

Fig. 9. Block diagram of the proposed long path $\mathrm{SO}_{2}$ monitor. 
wavelength $\lambda_{2}$ corresponding to a maximum in the sulfur dioxide absorption spectrum and the measurement process repeated. The number of wavelength pairs sampled would depend upon the severity of the interference problems encountered, The absorption spectrum of sulfur dioxide gas in the $300 \mathrm{~nm}$ region is shown in Figure 10,36 and as can be seen, rough1y 20 absorption 1 ines exist which would be suitable for use in making multiple wavelength measurements. Moreover, the interline spacing of roughly $2 \mathrm{~nm}$ suggests that 5 absorption 1 ines might 1 ie within the $20 \mathrm{~nm}$ lasing range of a dye such as rhodamine $6 \mathrm{G}$. To determine the wavelength interval between the peaks and valleys of a particular absorption line, one must use high resolution absorption spectra such as shown in Figure $11 .^{6}$ As can be seen, only a few tenths of a nanometer separate the peaks and valleys of a particular absorption line. As can also be seen, to fully utilize the interference rejection potential provided by such a narrow wavelength interval, the linewidth of the dye laser should be on the order of 10 percent or less of the peak-to-valley wavelength interval, and the wavelength stability of the laser should be similarly tight. It should be noted that high resolution absorption spectra were not available at the time the dye laser was being constructed, and therefore It was not possible to establish the necessary tolerances on the laser's spectral spread and wavelength stability.

A block diagram of the detection electronics is shown in Figure 12. A fraction of the output pulse falls on the reference detector while the remainder of the pulse proceeds on to the telescope. The peak output from the photodetector will be detected and retained 


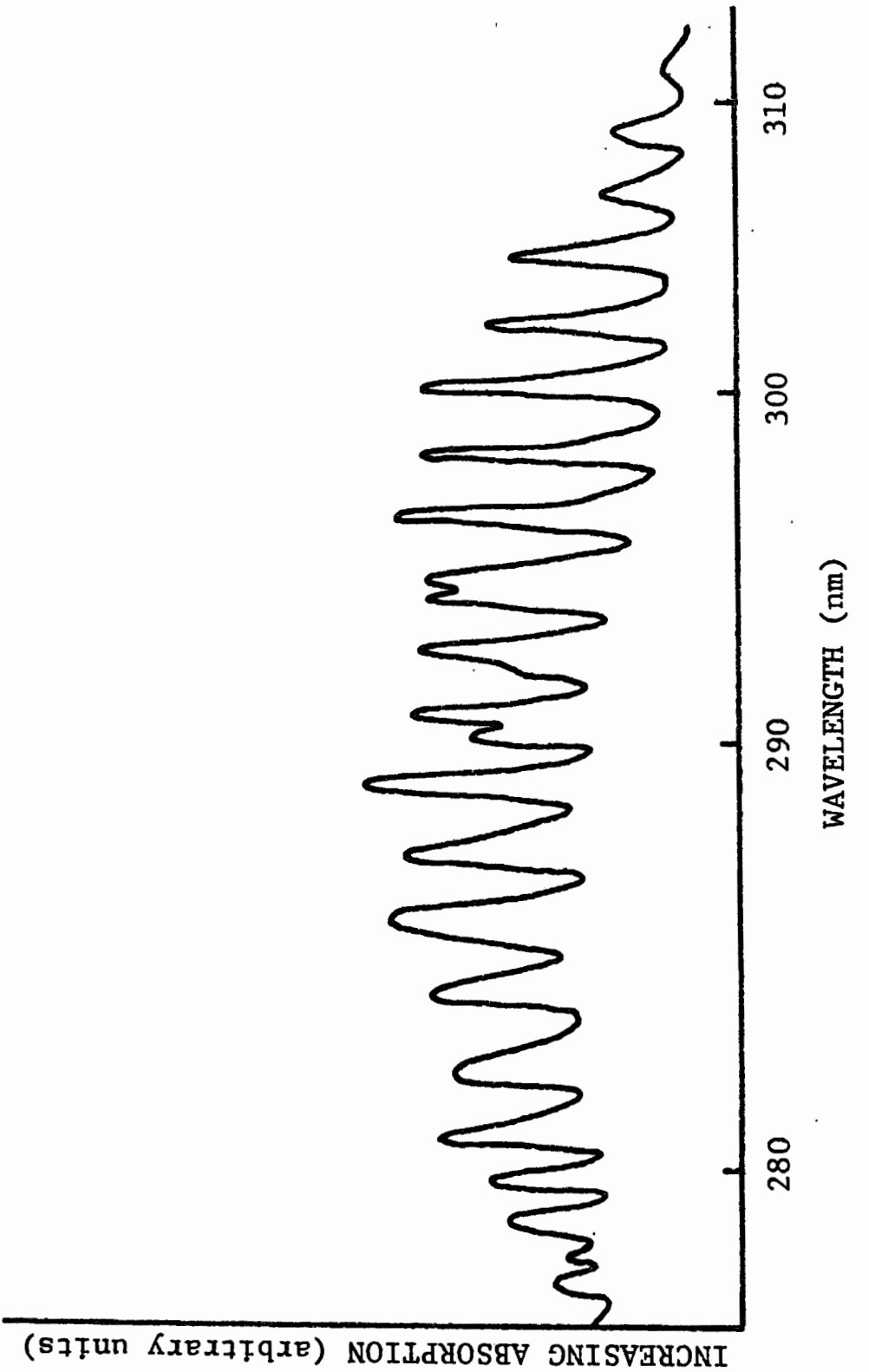




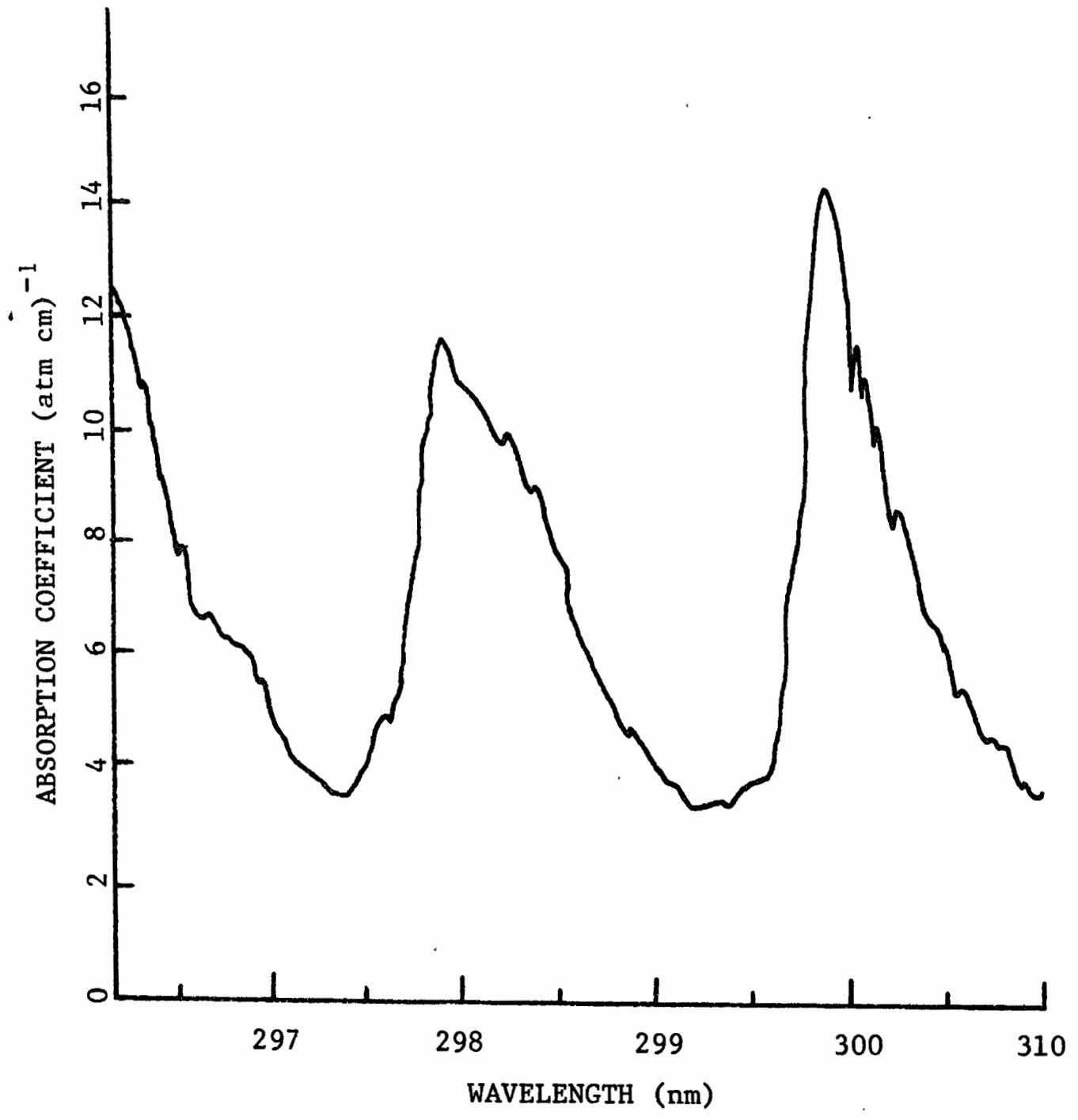

Fig. 11. High resolution absorption spectra of sulfur dioxide gas. (Ref. 6.) 


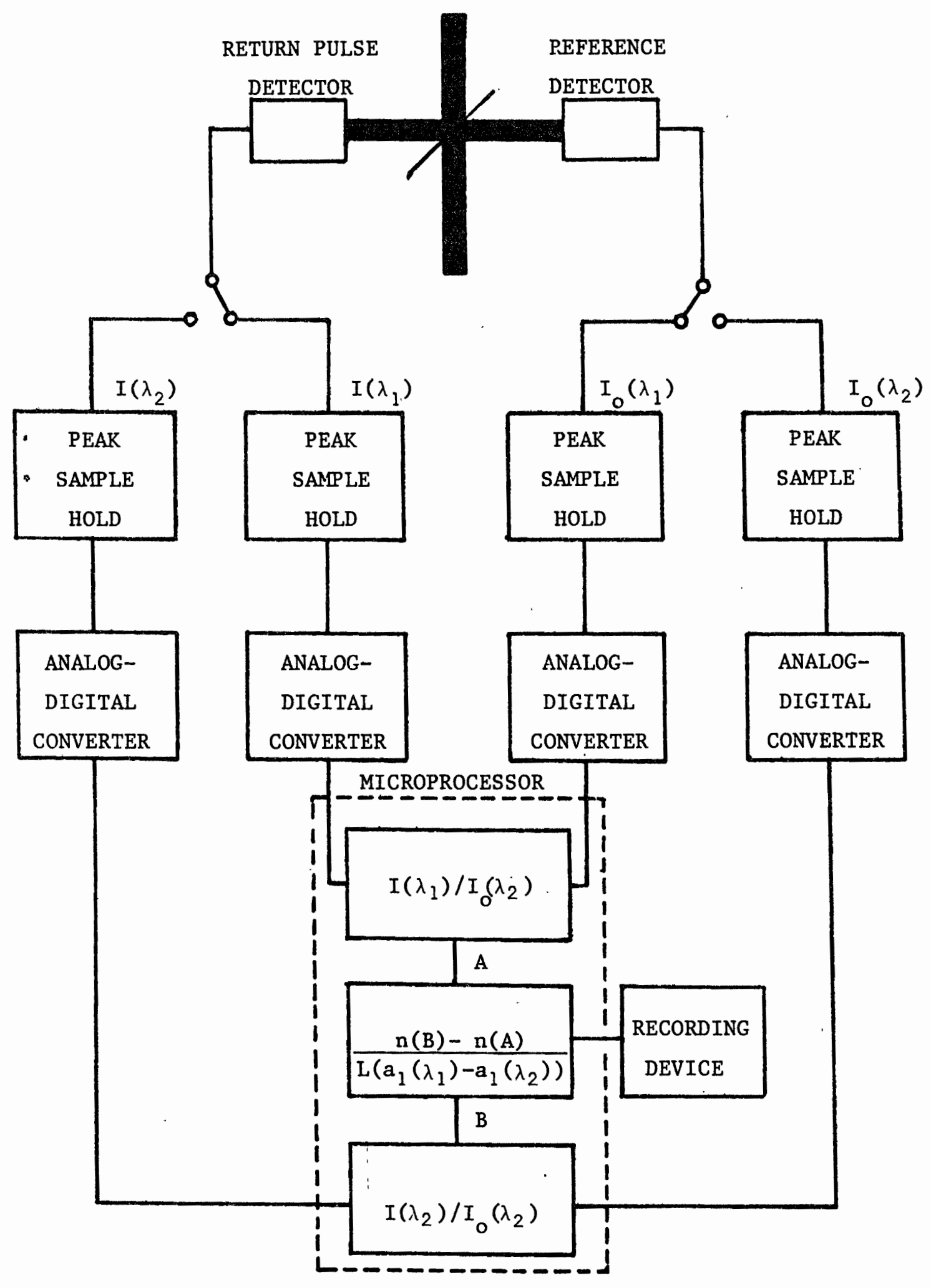

Fig. 12. Block diagram of detection electronics. 
by the peak sample and hold module. This step is necessary because analog to digital converters, the next step in the detection process, require on the order of $40 \mathrm{Hs}$ to convert an analog voltage level to a digital output, ${ }^{74}$ and therefore cannot be directly tied to the photodetector whose output will only be a few microseconds 1ong. Digital conversion is highly desirable because signals which are stored in digital form are inherently insensitive to problems which plague ana$\log$ storage devices such as storage capacitor leakage and finite amplifier input impedances. All four of the peak sample hold modules and analog to digital converters are identical and it is not necessary to describe the other three. The digital output from the A/D converters is stored by the microprocessor and, in the case of a two wavelength measurement, when the digital values for $I_{0}\left(\lambda_{1}\right), I\left(\lambda_{1}\right), I_{0}\left(\lambda_{2}\right)$, and $I\left(\lambda_{2}\right)$ have been stored, the concentration can be calculated by the microprocessor using (2.5). This final value for the pollutant's concentration can be visually displayed using a simple digital readout, or stored on a permanent recording medium such as magnetic tape. This completes the description of the optics used to transmit and receive the laser beam and the electronics necessary to detect the laser beam and turn the information contained in the optical pulse heights into an output indicative of the sulfur dioxide concentration over the long path.

The block diagram for the frequency-doubled flashlamp-pumped dye laser which was to serve as the ultraviolet light source for the longpath sulfur dioxide monitor is shown in Figure 13. The electrica1 energy necessary to power the dye laser is produced by a high voltage 


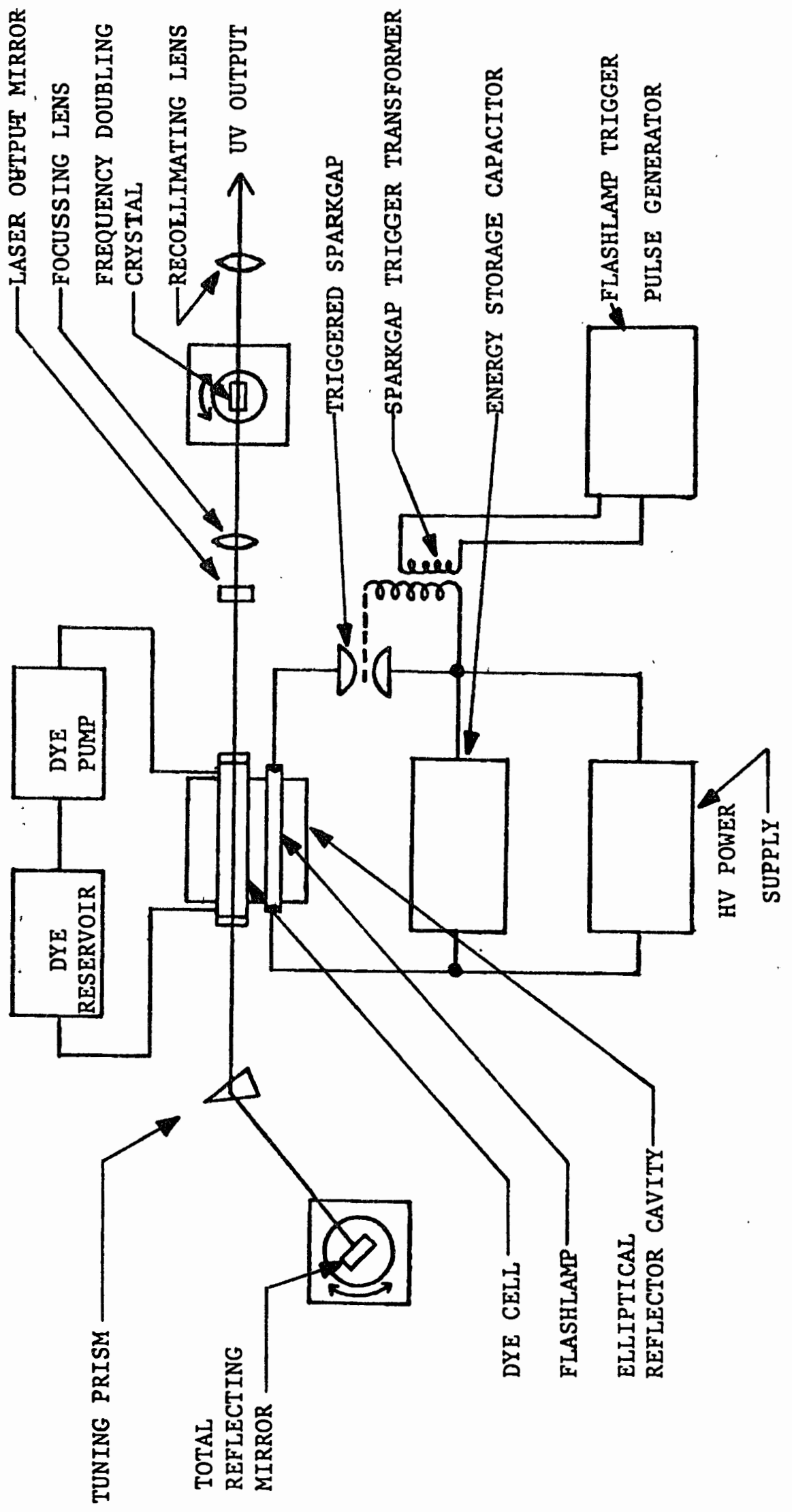

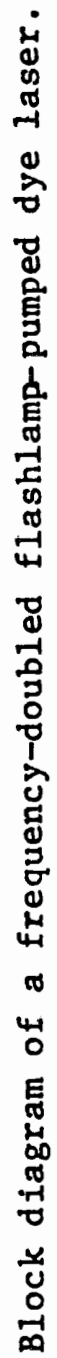

$m$
-1
0
0
01 
D. C. power supply and is stored in a high voltage energy discharge capacitor. The capacitor is usually one especially designed for discharge service, being capable of being rapidly discharged. When the capacitor is fully charged and a laser pulse is desired, the switch connecting the energy storage capacitor to the dye laser flashlamp is closed. The switch must be capable of holding off potentials of many thousands of volts, and when closed pass currents of several thousand amps. In practice it is typically in the form of a triggered spark gap, although hydrogen thyratrons have also been used. Current supplied by the capacitor flows through the gas in the flashlamp, heating the gas to temperatures which can reach many thousands of degrees Kelvin. The flashlamp and a flowtube containing the dye are generally placed at the opposite foci of an elliptical cavity such that light from the hot gas within the flashlamp is focussed by the reflective elliptical surface onto the glass tube containing the laser dye. That portion of the flashlamp light which lies within the absorption spectrum of the dye excites the dye molecules which become de-excited by radiating light in the form of fluorescence. If the flashlamp pulse is strong and a large enough number of the dye molecules are excited, lasing can occur. The dye cell is placed between two mirrors forming what is termed the laser resonator. In order for 1 ight to repeatedly pass through the dye cell and be amplified, the two mirrors which form the resonator must be aligned such that their reflecting surfaces are perpendicular to a cormon axis which passes through the dye medium. The angular tolerance on the mirror alignment is on the order of a fraction of a milliradian, a number which points to the need for 
mirror mounts whose angle can be precisely and smoothly adjusted. The dye 1aser. output wavelength can be tuned by inserting a prism between the totally reflecting mirror and the dye cel1, in which case tuning is accomplished by rotating the totally reflecting mirror. A second method is to replace the totally reflecting mirror with a diffraction grating which is a!so tuned via rotation. In the proposed pollution monitor, the rotation of the tuning element was to be accomplished via a stepping motor driven screw which would push against a lever arm which would in turn rotate the tuning element as shown in Figure 14 . The precise amount of table rotation will depend upon the number of voltage pulses fed into the stepping motor since the stepping motor turns a precise amount for each applied pulse. The number of voltage pulses necessary to tune the dye laser to a wavelength corresponding to a pollutant absorption line is a quantity which can be stored in the memory of a small and increasingly inexpensive minicomputer. The same minicomputer can be used to control the firing of the laser and process the received data. With the addition of a pump to circulate the dye through the dye cell, the list of major components necessary to produce pulses of tunable visible light is complete.

All that is required to convert visible light to ultraviolet light, and therefore complete the light source for the pollutant monitor, is a frequency doubling crystal. For the conversion to be efficient, the intensity of the visible light should be on the order of 10 to 100 megawatts per square centimeter. Since few lasers produce pulses of such intensity directly, it is usually necessary to focus the light into the frequency doubling crystal with a lens, and then 


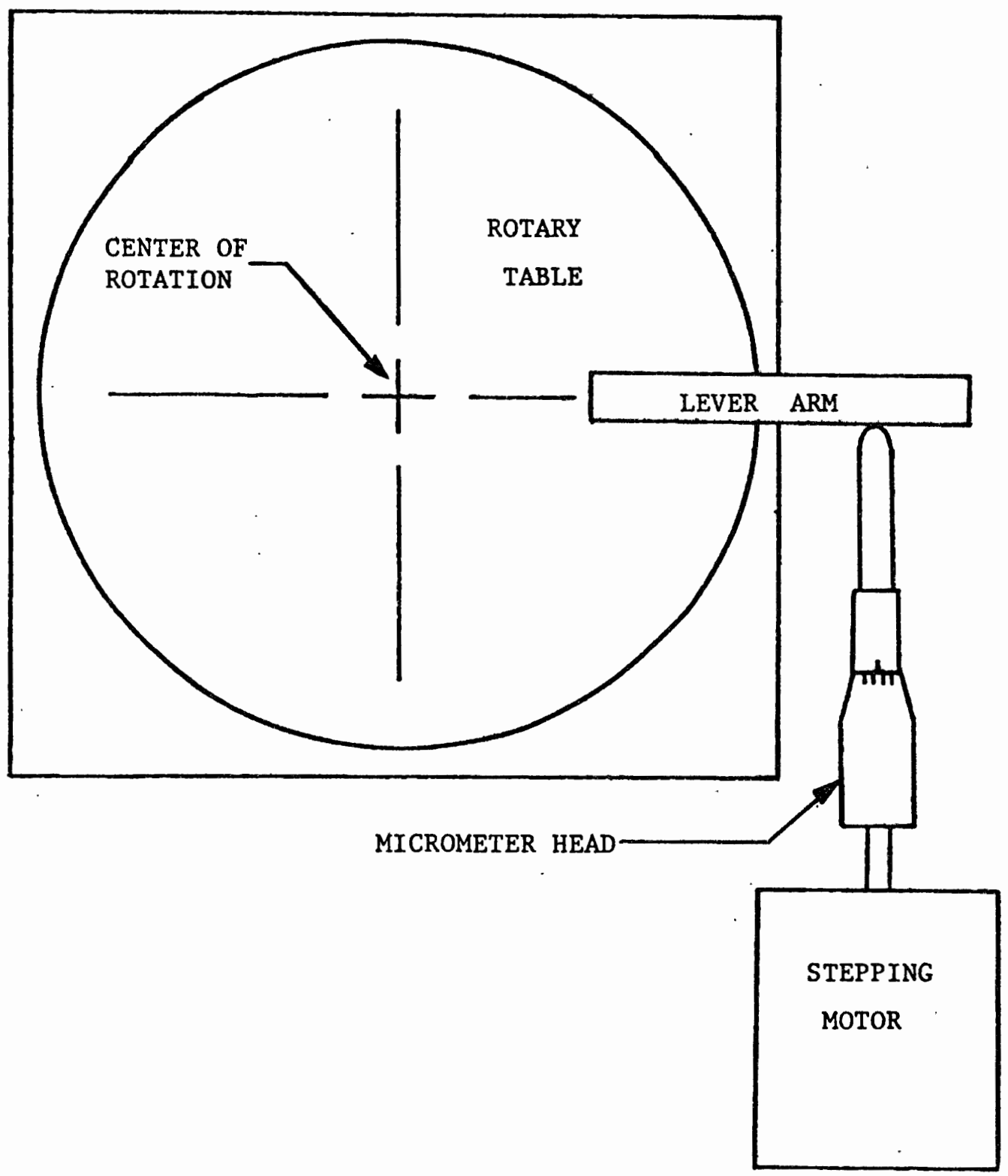

Fig. 14. Simplified rotational mount used to automatically rotate tuning elements. 
recollimate it with a second lens after it has left the frequency doubler. As will be shown in a later chapter, the frequency doubling crystal must be precisely oriented to a particular angle with respect to the incoming beam if efficient second harmonic generation is to occur, and it will be necessary to change the angle each time a different wavelength is desired. This adjustment can be performed by a stepping motor driven rotary table similar to the one described previously for tuning the dye laser.

The non-laser portion of the pollutant monitor optics would consist of a small telescope to expand the laser beam to the diameter necessary for propagation over the desired path, and a remote retroreflector to return the transmitted beam to the telescope for collection. The actual detection of the returned pulses could be accomplished either with a photomultiplier or a solid state photodiode. From that point the problem is one of computing the pollutant concentration from the detector outputs.

In the next two chapters the dye laser and frequency doubler will be more thoroughly analyzed and construction details given. In the last chapter the performance of the frequency-doubled flashlamppumped dye laser will be detailed. 
CHAPTER VI

DESIGN AND CONSTRUCTION OF A FLASHLAMP-PUMPED DYE LASER

In this chapter the design and construction of the flashlamppumped dye laser will be detailed. The first section is a descriptive one and describes the energy transfer processes which take place within an excited dye molecule. The purpose of the section is to demonstrate the need for a short duration flashlamp pulse. In the second section an estimate of the pulse length which might be obtained from a realistic flashlamp is made. Section three describes the actual construction of the flashlamp system and compares its operation with the analytical results of the previous section. The fourth section deals with the optical cavity used to couple the light from the flashlamp into the dye cell and also briefly describes the pump system used to circulate the dye. Section five describes the components of the laser resonator and examines both analytically and experimentally the use of a diffraction grating and a prism as tuning elements for the dye laser. In the final section a method for rapidly tuning the dye laser to more than one wavelength within a single dye laser pulse will be described.

\section{Energy Transfer Pathways in Excited State Dye Molecules}

The organic dyes which are the active medium in the dye laser are typically a multi-ring organic compound with substitutional groups positioned about the perimeter of the basic compound. Virtually all of the dyes in use today originate from one of three basic chemical 
compounds: the coumarins, the xanthenes, and the oxazines. 75

The absorption and emission spectra of organic dyes are typical1y very broad functions of wavelength as opposed to the narrow absorption spectra obtained from gases and solids (see Fig. 15). The reason is that unlike most solids and gases, organic dyes because of their relatively complicated chemical structure, have a large number of vibrational and rotational modes possible. A typical dye molecule with 50 atoms might possess 150 vibrational modes, with each mode contributing a small amount of energy $\Delta \mathrm{E}_{\mathrm{v}}$ on top of the energy due to the excited electron. In addition to the closely spaced vibrational transitions, there are a large number of even more closely spaced rotational transitions between each vibratonal transition. The result is a virtual continuum of transitions producing a broad absorption and emission line. In a dye laser, dye molecules are pumped to a highlying singlet state from the ground state by absorbing a photon of pump light (see Fig. 16). These excited state molecules rapidly lose energy through radiationless transitions until they reach one of the lower lying levels of the first excited singlet state. Once in the first excited singlet state, the dye molecule may return to the ground state through one of two main routes. The preferred route is through fluorescence. In the laser dye rhodamine 6G, 85 percent of the excited singlet state molecules can return to the ground state via the fluorescence mechanism. 69 The second non-emitting process by which excited state molecules can return to the ground state is via a slow two step process through the triplet state. Transitions to the triplet state from the singlet state, and from the triplet state to the 

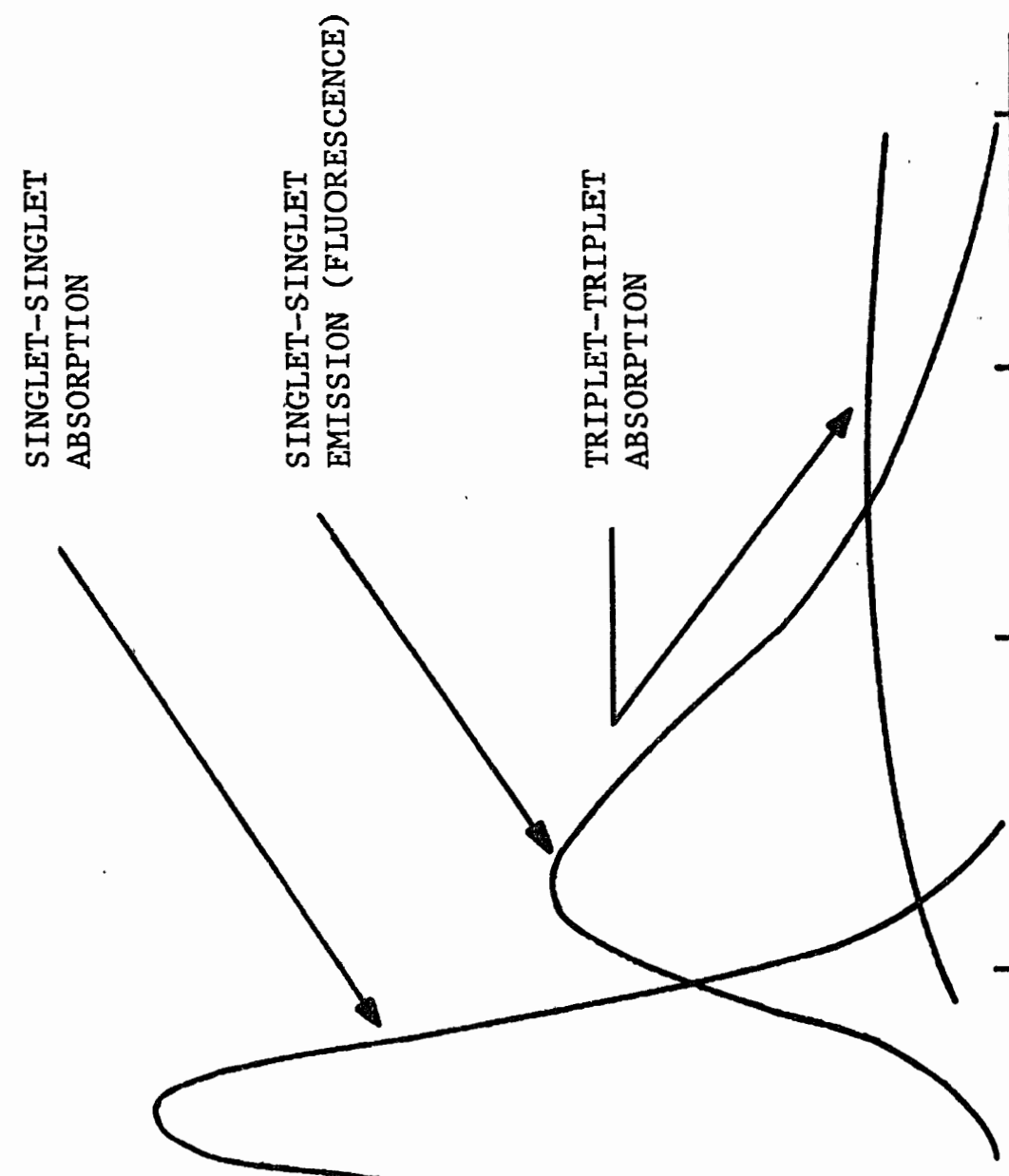

8

㤩

s.

年4

出

己ृ.

峁

$\rightarrow 0$

号

क क

山ै

-1

点

望|

XIISNGINI NOISSIKH YO NOILdYOSGV DNISVGYONI 


\section{HIGH LYING \\ EXCITED SINGLET}

STATE LEVELS

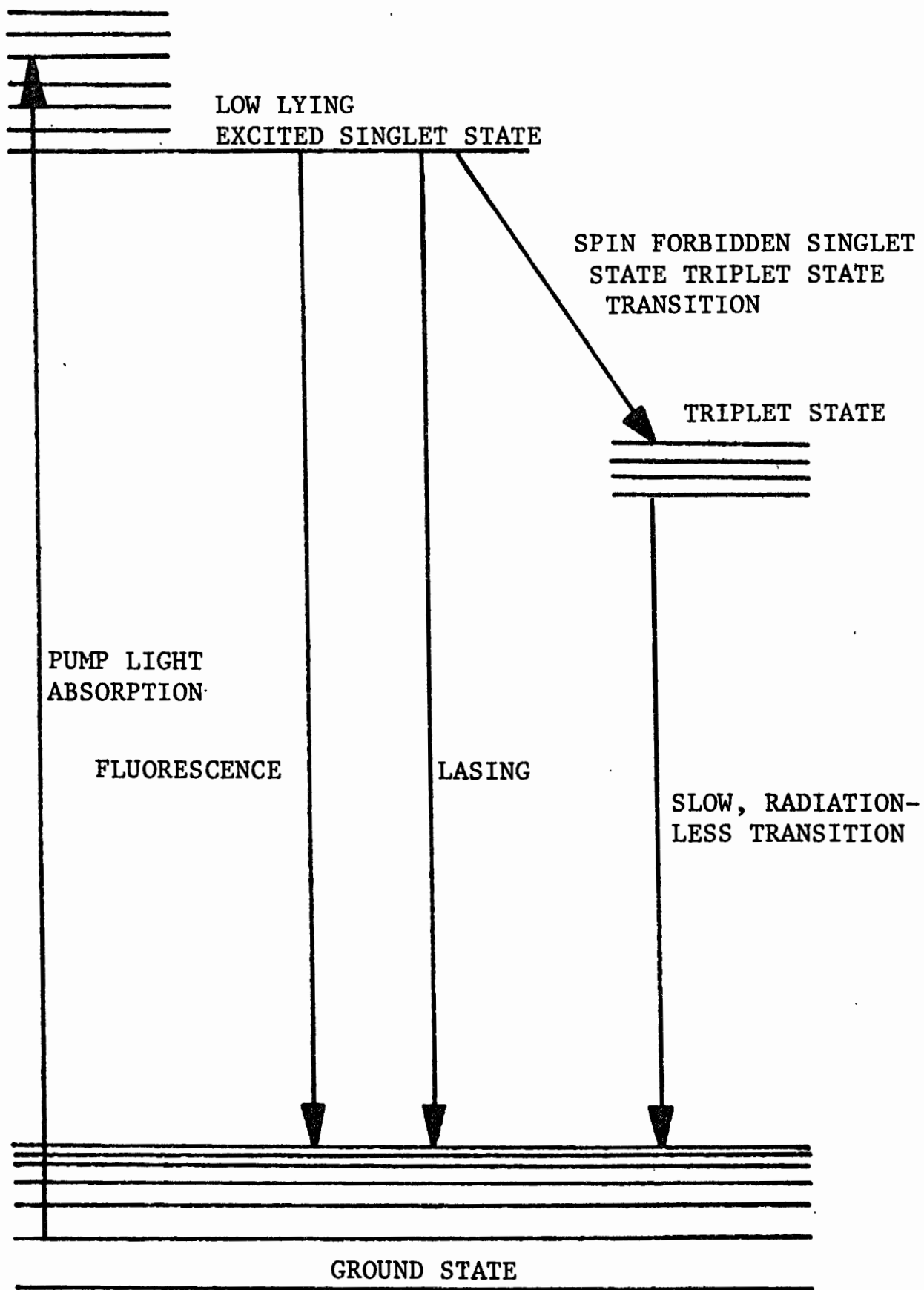

Fig. 16. Energy levels and transitions relevant to an organic dye laser. 
ground state occur at a very slow rate since they are spin forbidden. ${ }^{79}$ Those dye molecules which do end up in the triplet state consitute a severe optical loss mechanism in a dye laser, since the triplettriplet absorption spectrum overlaps the dye laser's output spectrum. In pulsed dye lasers having pulse lengths longer than a few hundred nanoseconds, the triplet state absorption losses can become so large as to actually cause the dye laser to cease lasing. ${ }^{76}$ A number of additives have been found to increase the triplet state to ground state transition rate, ${ }^{77-79}$ and thus allow long pulse ( $\left.250 \mu s\right)$ operation of flashlamp-pumped dye lasers with a number of dyes spanning the visible region of the spectrum. However for one reason or another, triplet state quenching agents, other than the normally present amount of dissolved oxygen, are not widely used. One possible reason for the lack of use of one such additive, cyclooctatetraene (COT), may be chemical instability. In our laboratory an unopened bottle of cOT was found to have decomposed after less than one year even though it was not subjected to temperature extremes or high light levels. The usual practice for reducing triplet state effects is to use as short an optical excitation pulse as is practical, a practice which was followed in designing the flashlamp system for our dye laser. Early analytical studies of the effect of flashlamp pulse duration on dye laser operation indicate that triplet state losses can prevent lasing if the flashlamp pulse is not intense enough to promote lasing within the first microsecond after the initiation of the flashlamp pulse. 77 Moreover, since triplet state losses will continue to accumulate throughout the duration of the flashlamp pulse, thus degrading the 
dye 1aser's efficiency, a wise strategy is to employ as short a flashlamp pulse as possible. In the next section an estimate of the expected flashlamp pulse length will be made.

\section{Analysis and Design of Flashlamp System}

Because of the required short duration of the pumping pulse it is important to calculate the pulse length which might come from a realistic flashlamp pumping system. Such a system is shown in Figure 17. In this system electrical energy is stored by charging a low inductance energy discharge capacitor to a high voltage, typically 10 to $40 \mathrm{kV}$. The charging resistor prevents the high voltage supp $1 \mathrm{y}$ from providing enough current to hold the lamp and sparkgap in conduction once triggered. Once the capacitor is fully charged, it is connected to the flashlamp using a triggered sparkgap. The flashlamp goes from a non-conducting state to conducting state almost instantly, since the applied voltage of 10 kilovolts or more is much higher than the self-breakdown voltage of a flashlamp filled with a noble gas ( $500 \mathrm{~V} /$ inch). 80 The high current which flows during the discharge creates an extremely hot plasma inside the flashlamp. The plasma emits light in a spectral distribution which can be closely approximated by a blackbody. 81 Because of the relatively high temperature found in high current density plasmas, the resultant emission is peaked in the near ultraviolet and visible spectral regions where the absorption bands of laser dyes appear. If one neglects the inductance of the capacitor $\left(\sim 1.5 \times 10^{-8} \mathrm{H}\right)^{82}$ and the capacitance of the flashlamp leads ( $\left.10^{-12} \mathrm{~F}\right),{ }^{83}$ then one can use the greatly simplified 


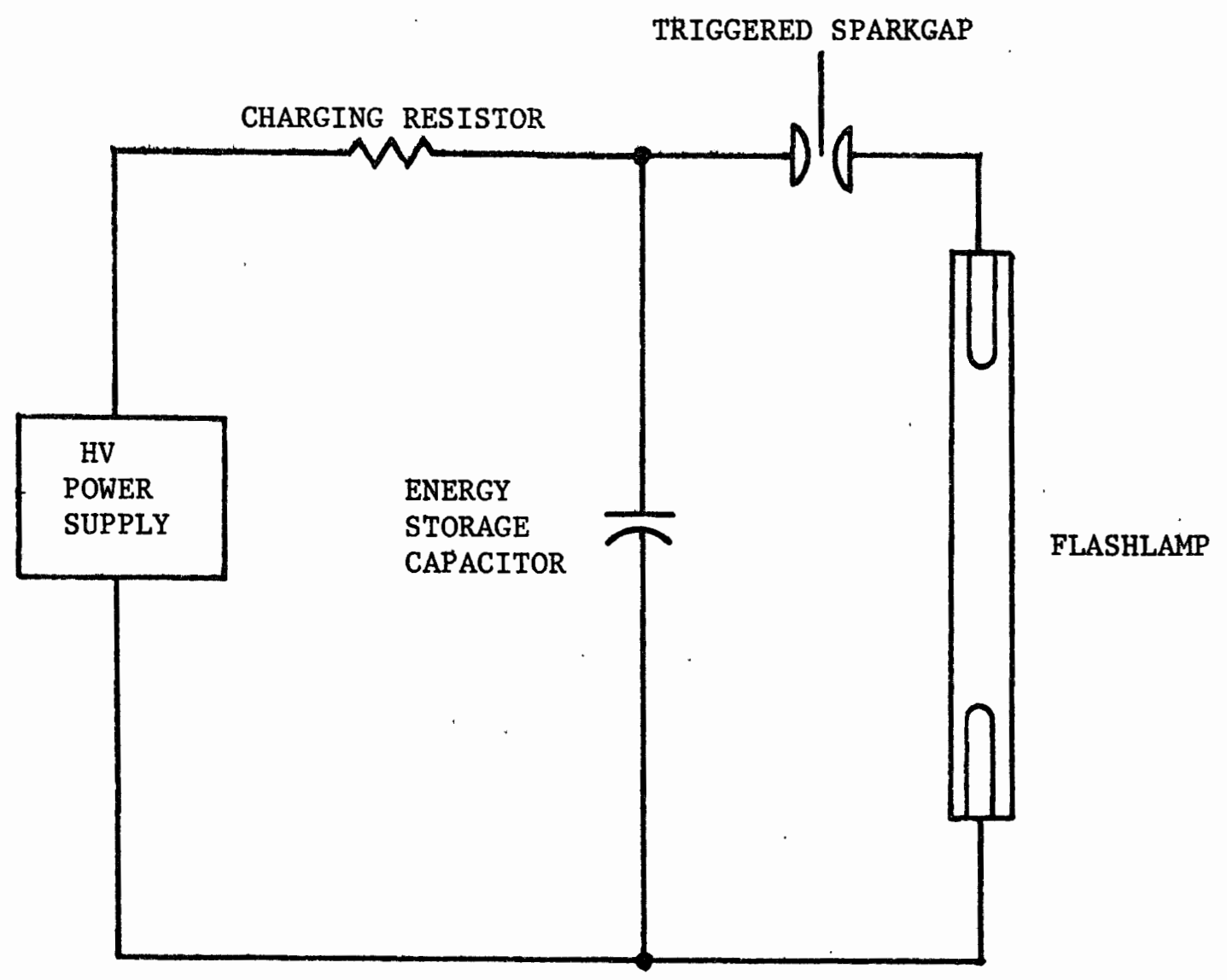

Fig. 17. Simplified diagram of a capacitor discharge powered flashlamp pumping system. 
equivalent circuit shown in Figure 18 to calculate the flashlamp pulse length.

The inductance of the leads can be approximated by assuming the circuit to be a two wire transmission line. The inductance of a two wire transmission line of length $\ell$ is given approximately by: ${ }^{83}$

$$
L \sim \frac{\mu_{0}}{\pi} \ln \left(\frac{d}{a}\right) \ell
$$

where:

$$
\begin{aligned}
& \mu_{0}=\text { permittivity of free space } \\
& \mathrm{d}=\text { the wire spacing } \\
& a=\text { the wire radius } \\
& \ell=\text { the length of the transmission line. }
\end{aligned}
$$

Using realistic values of $2.5 \mathrm{~cm}$ for $\mathrm{d}, 0.25 \mathrm{~cm}$ for a, and a length of $15 \mathrm{~cm}^{84}$ we arrive at a value of $0.14 \mu \mathrm{H}$ for the estimated circuit inductance. This value of the inductance is much larger than that associated with the capacitor and can probably be safely used as a first order estimate for the total circuit inductance.

The value of the energy storage capacitor in the circuit was determined from energy storage considerations. The energy stored by a capacitor is given by: ${ }^{7}$

$$
\mathrm{E}=\frac{1}{2} \mathrm{CV}^{2}
$$

where

$$
\begin{aligned}
& E=\text { the energy stored in the capacitor } \\
& C=\text { the capacitance of the capacitor } \\
& V=\text { the voltage across the capacitor. }
\end{aligned}
$$

It was felt that the energy storage capacitor should have the 


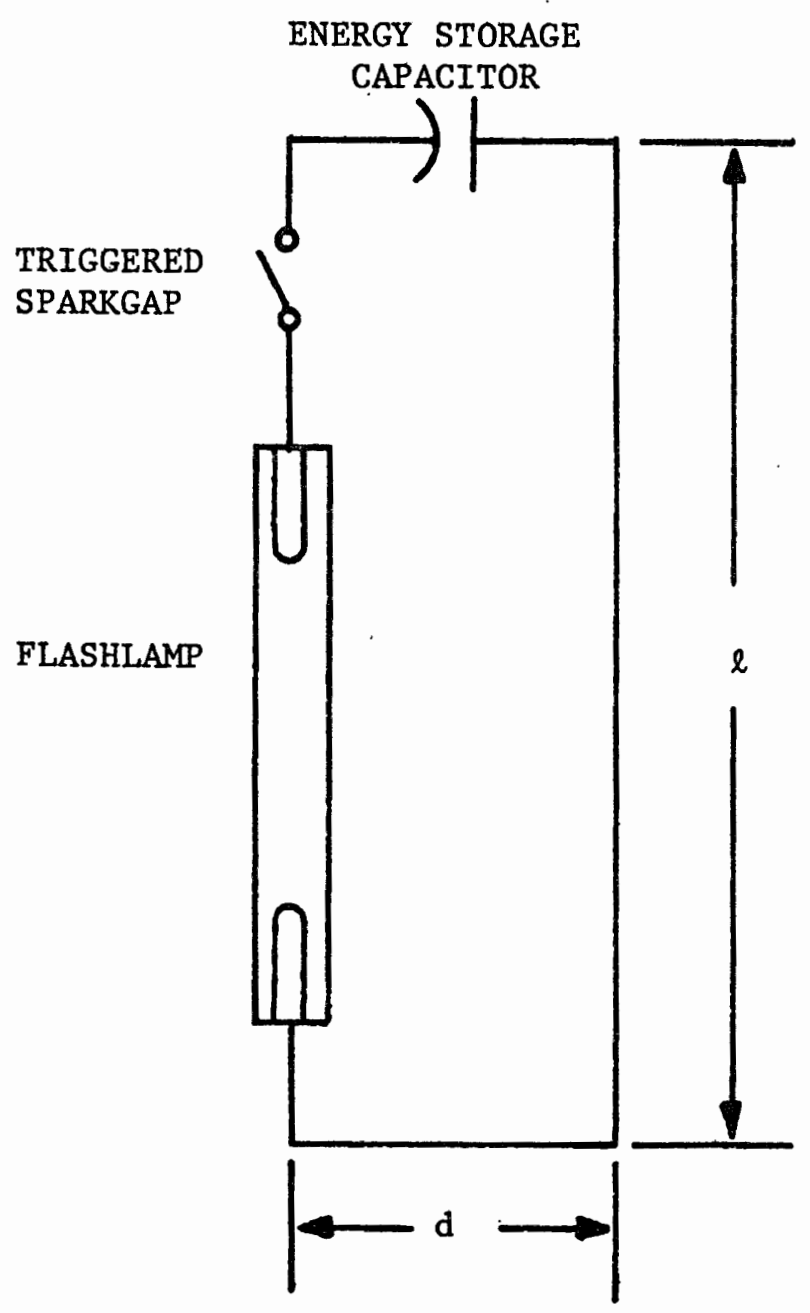

Fig. 18. Simplified flashlamp pumping system showing only those components and parameters used in estimating the flashlamp pulse length. 
capability of delivering 100 Joules of electrical energy to the flashlamp. It was also felt that 20,000 volts represented an upper bound on our high voltage capability, since beyond this voltage the problems of corona and breakdown rapidly increase making construction more difficult. Inserting these values of $E$ and $V$ into (6.2), one arrives at the required value of capacitance of $0.5 \mu \mathrm{F}$.

The quantities necessary for the calculation of the excitation pulse duration have now been determined, except for the resistance in the circuit. The resistance associated with the flashlamp is a highly variable function of the gas in the lamp, the lamp diameter, etc. From experience, the resistance of a conducting flashlamp is such that it will typically produce a slightly underdamped discharge, the pulse length of which will be on the order of: ${ }^{7}$

$$
\tau_{p} \simeq \pi \sqrt{L C}
$$

Using our previously determined values for $\mathrm{L}$ and $\mathrm{C}, 0.14 \mu \mathrm{H}$ and $0.5 \mu \mathrm{F}$ respectively in (6.3), one gets a pulse length of $\tau_{p} \sim 0.83 \mu \mathrm{s}$. This calculated pulse length is in agreement with experimentally determined pulse lengths from similar systems, and is less than one microsecond long, which means that the triplet state losses should not become extremely large.

It is also interesting to calculate the damping factor for the discharge. It is this factor which will indicate whether the LC discharge is underdamped, critically damped, or overdamped. A critically damped pulse will deliver the greatest amount of energy to the flashlamp in the least amount of time. ${ }^{86}$ For short pulse flashlamps such 
as used in this laser, the damping factor is given by: 86

$$
\alpha=\frac{1.2 \ell}{d}\left[\frac{\mathrm{C}^{2}}{2 \mathrm{EL}}\right]^{1 / 4}
$$

where $l$ and $d$ are the flashlamp's length and diameter in consistent units. Evaluating (6.4) for a flashlamp length of $7.5 \mathrm{~cm}$, a diameter of $0.2 \mathrm{~cm}$, and using the previous values for capacitance, inductance, and stored energy, we get a value for $\alpha$ of rough1y 0.5 . This is less than the value for a critically damped pulse $(\alpha=0.8), 86$, and means that the current pulse from the capacitor will tend to be underdamped. The amount of underdamping is smal1, however, and the capacitor will still be able to deliver 90 percent of its energy to the lamp within thé calculated pulselength of 0.83 microseconds. For flashlamp lifetime reasons which will be detailed in a later section, the lamp was normally operated at energies much lower than the $100 \mathrm{~J}$ value used in calculation, a factor which would yield a damping factor closer to the ideal, critically damped value of 0.8 .

\section{Flashlamp Driver System}

A flashlamp driver system very similar to the one just described was constructed for use with the dye laser. In this section a more detailed description will be given of the high voltage power supply, the energy storage capacitor, the triggered sparkgap and its associated trigger circuitry, and finally the flashlamp itself.

High Voltage Power Supply. The high voltage power supply used to charge the energy storage capacitor was specially constructed at Portland State University, and a simplified schematic diagram is shown in Figure 19. Line voltage of 115 VAC was first applied to the input 

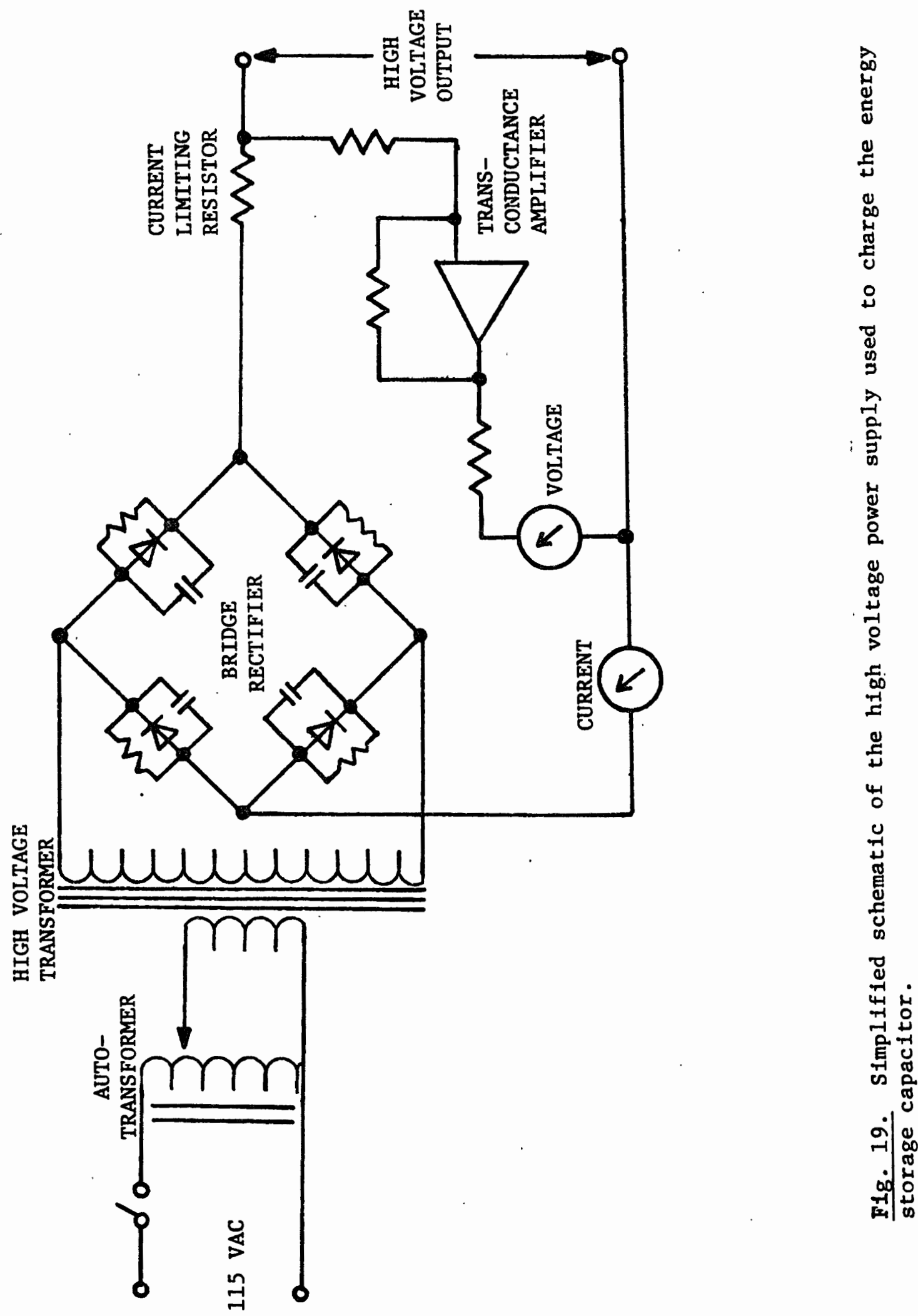
windings of a $10 \mathrm{~A}$ RMS autotransformer. The variable voltage output from the autotransformer secondary was connected to the primary side of a specially constructed high voltage step-up transformer forming the basis for a variable high voltage power supply. The step-up transformer had a primary-to-secondary turns ratio of $1: 123$ and used paper and oil insulation. The high voltage A.C. from the transformer was rectified using bridge arrangement, each leg of which had forty, $800 \mathrm{~V}, 1 \mathrm{~A}$ silicon diodes in series. Each diode was paralleled by both a $1 \mathrm{~m} \Omega, 2 \mathrm{~W}$ resistor to insure that the high voltage would be equally distributed across each of the diodes, and a $.01 \mu \mathrm{F}, 1000 \mathrm{~V}$ ceramic disc capacitor to bypass any fast transients which might also damage the diodes. Two identical, large-face panel meters were used to monitor the output voltage and output current from the power supply. The meters had a full scale deflection sensitivity of $100 \mathrm{~mA}$ and could be used for the current monitoring function directly. For voltage monitoring the meter would have consituted an unreasonably large drain upon the supply. This problem was overcome by using an operational amplifier as a current amplifier to provide the necessary current boost. A $20 \mathrm{k} \Omega, 40 \mathrm{~W}$ resistor was used as a current limiter in the output high voltage lead.

Energy Storage Capacitor. The energy storage capacitor had a capacitance of $.05 \mu \mathrm{F}$ and could store $100 \mathrm{~J}$ of energy at its rated voltage of $20 \mathrm{kV}$. It was purchased from Condenser Products and had an internal inductance of $15 \mathrm{nH}$ according to its manufacturer.

Triggered Spark Gap. The triggered sparkgap which was constructed is shown in Figure 20, and was designed to fit coaxially 


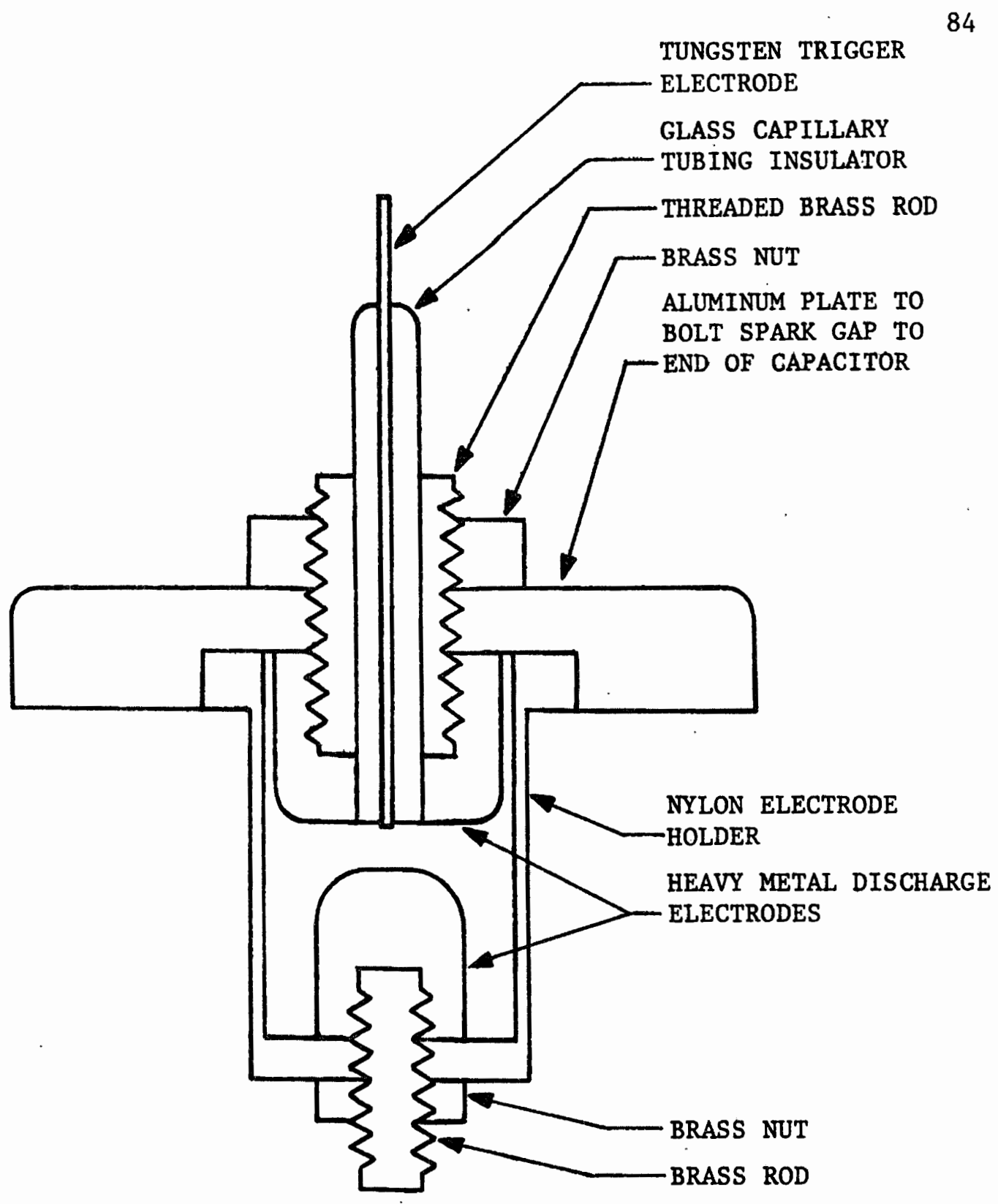

Fig. 20. Diagram of spark gap used to fire laser. 
inside the cylindrical capacitor, thus minimizing the inductance of the circuit. The main discharge electrodes were constructed of a tungsten alloy called "heavy metal" supplied by Mallory Metals, Inc. The tungsten alloy is a product of powder metallurgy technology and is composed of 94 percent tungsten powder held together in 4 percent copper -2 percent nickel "binder." The end product is a material highly machinable while retaining the refractory properties of pure tungsten. The trigger electrode was thoriated tungsten wire which was insulated from the main discharge electrode by passing it through the center of a piece of $6 \mathrm{~mm}$ O.D. capillary glass tubing. Electrical connection to the trigger wire was by means of a small solder lug which was silver soldered to the trigger wire. The holdoff of the gap could be adjusted by varying the sparkgap's main electrode spacing. The sparkgap proved to be one of the most reliable parts of the laser, requiring no maintenance other than occasional cleaning. The measured maximum hold off voltage and minimum triggerable voltage as a function of electrode spacing is shown in Figure 21.

The sparkgap was triggered into conduction by a high voltage pulse applied between the trigger electrode and the main electrode into which the trigger electrode coaxially fits. The high voltage trigger pulse was produced by discharging a $0.47 \mu \mathrm{F}$ capacitor charged to approximately $400 \mathrm{~V}$, through the primary of a step-up transformer which was very similar to an automobile ignition transformer (see Fig. 22). While it is possible to use a standard automobile ignition coil to trigger the sparkgap, the necessity of floating the secondary side of the transformer forced the winding of a special 


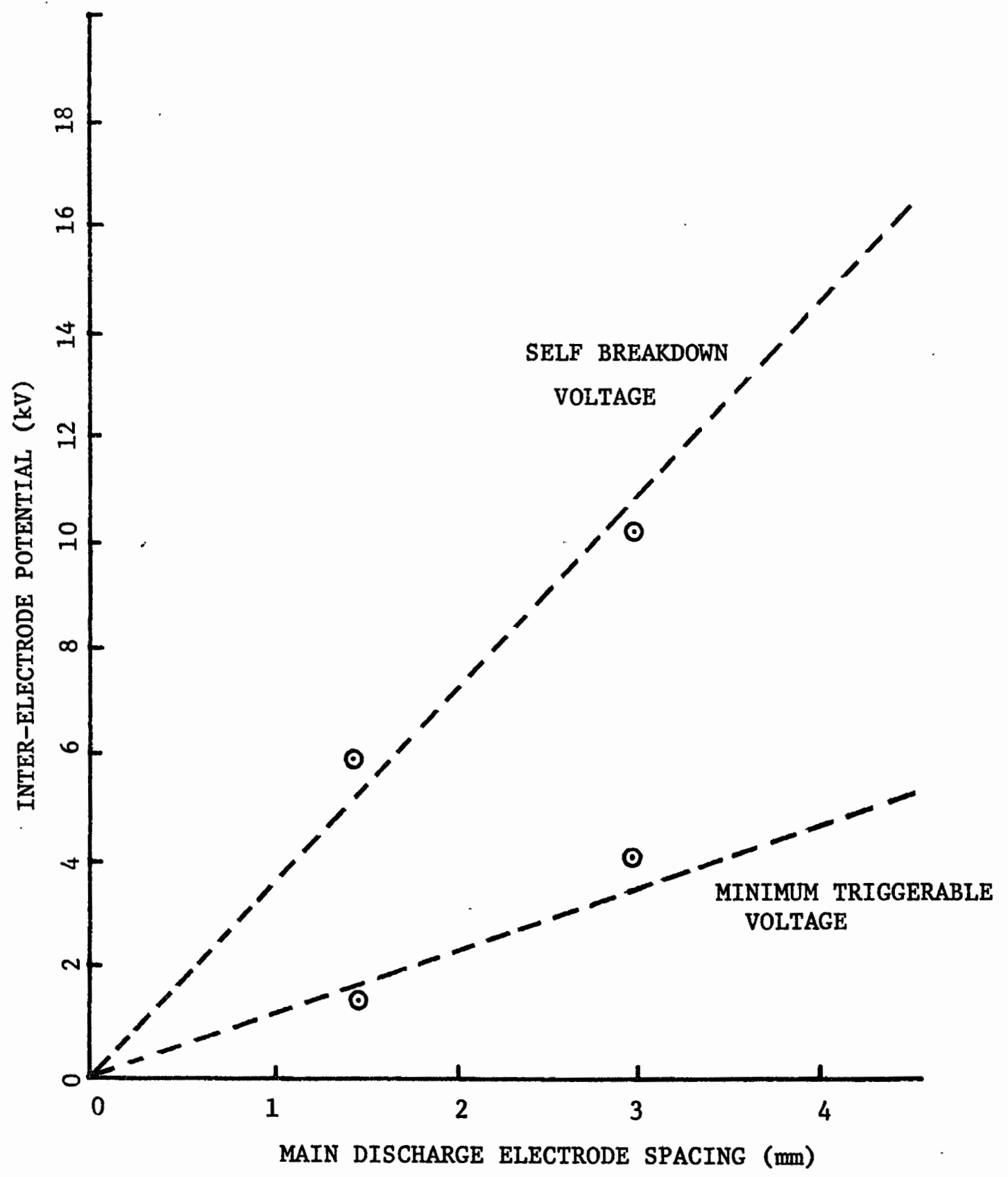

F1g. 2̀l. Breakdown characteristics of triggered spark gap. 


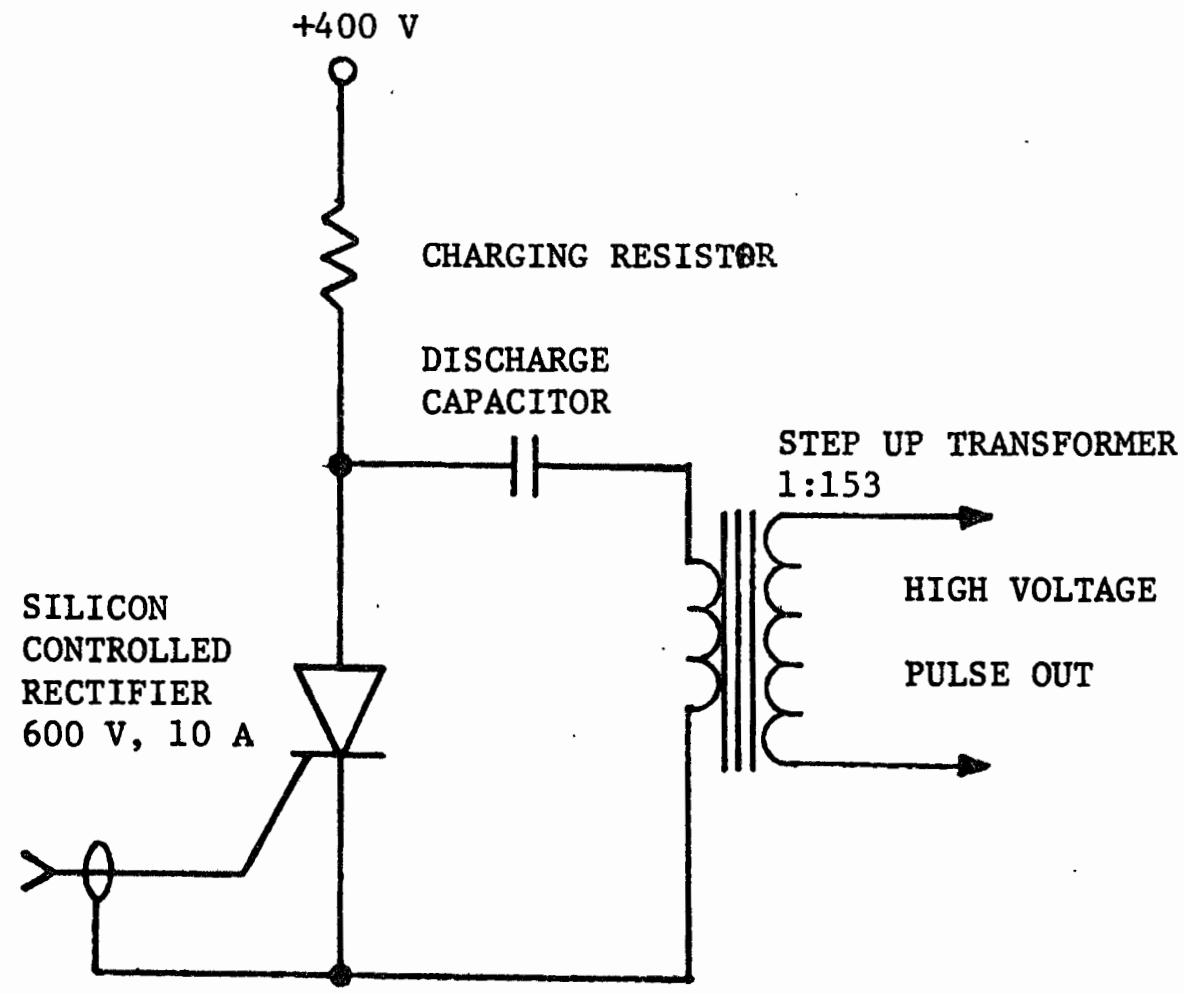

Fig. 22. Simplified schematic of the circult used to trigger the triggered spark gap. 
trigger transformer. The trigger transformer was wound on a sma11 epoxy-glass bobbin which was fabricated from printed circuit board. The primary consisted of 10 turns of number 14 magnet wire while the secondary consisted of 1530 turns of number 37 magnet wire, thus giving a step up ratio of 153 to 1 . After numerous attempts to pot the transformer in epoxy failed, it was finally decided to immerse the transformer in transformer oil. While this solution to the insulation problem is esthetically less pleasing than potting, it is virtually foolproof. In this way, $\sim 50 \mathrm{kV}$ trigger pulses for the sparkgap were obtained. The timing pulses to trigger the sparkgap were provided by an external pulse generator and the entire triggered sparkgap system was capable of operating at pulse rates of several $\mathrm{Hz}$.

Flashlamp Construction. The first flashlamps to be used in the dye laser had a $7.5 \mathrm{~cm}$ arc length and were constructed from $7 \mathrm{~mm} 0 . \mathrm{D}$. fused silica tube with $1 \mathrm{~mm}$ walls (see Fig. 23). The electrodes were machined from stainless steel rod. The flashlamp was operated with a low static pressure ( 5 Torr) air fill, and was evacuated through a sidearm on the cathode end of the lamp.

Several problems were noted in using the evacuated flashlamps. The first problem was the difficulty in sealing the electrodes to the quartz tubing. A11 attempts to epoxy the electrodes to the tubing failed, a factor attributable to the differences in thermal expansion between the stainless electrodes and the fused silica lamp jacket. When the electrodes were attached by wrapping a piece of tape around the flashlamp-electrode interface, the tape ruptured after a few shots due to the explosive high pressure shock wave generated during the 


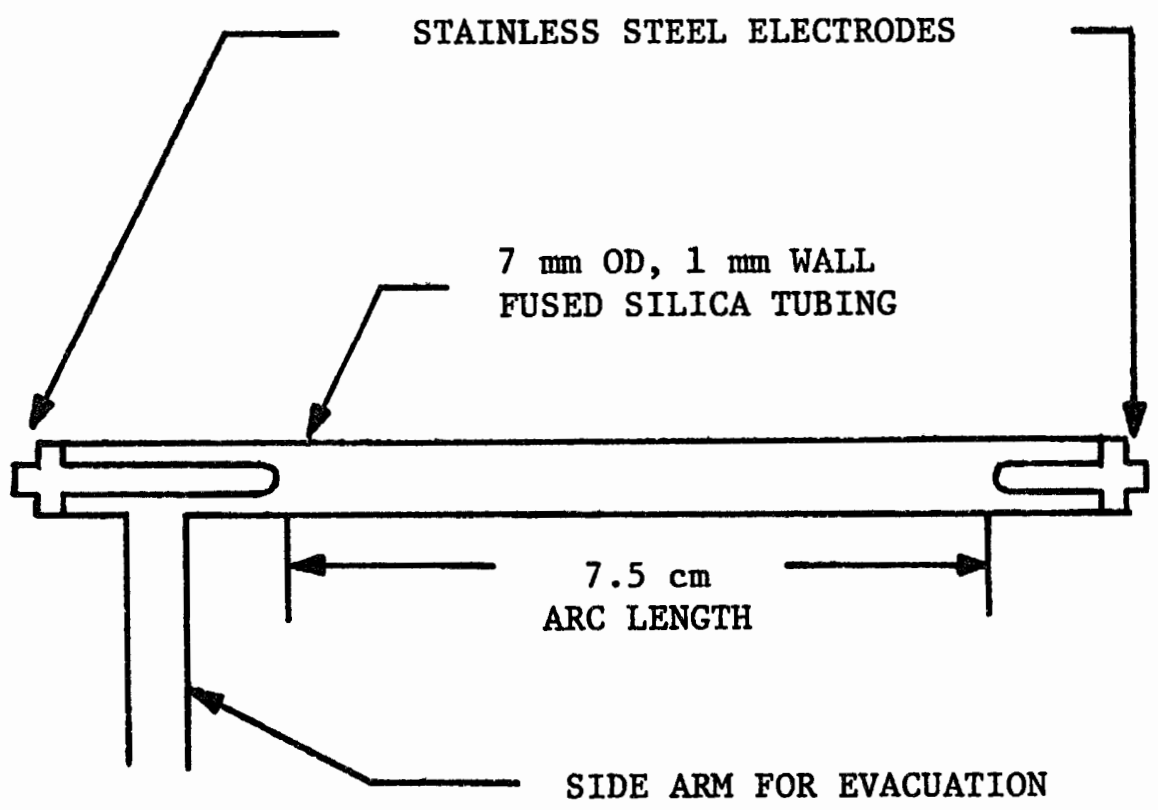

Fig. 23. Low pressure air filled flash lamp. 
discharge making it impossible to maintain a low pressure in the arc region.

A second problem was that the stainless electrodes experienced serious sputtering which would coat the inside of the quartz tube with ablated material. A number of different stainless steel electrode geometries were tried in an effort to alleviate the sputtering problem, however none of the designs proved successful. A more successful design used brass electrodes fitted with heavy metal tips on the discharge end. The tungsten tips eliminated much of the sputtering problem. A second deposit problem which was not alleviated by the tungsten electrodes was the buildup of what was assumed to be reduced silicon around the electrode. The silicon originates from the flashlamp wall, a small portion of which is ablated away on each shot. As will be noted later, this problem proved to be the ultimate lifetime limitation.

The major problem with the evacuated air flashlamps was their poor electrical to light conversion efficiency. Based on conversations with Neil Sandow of Chromatix and John Clark of the University of California at Berkeley, it was decided to switch to a flashlamp which used argon gas at one atmosphere instead of low pressure air. Argon, even at atmospheric pressure, can be easily made conducting by the application of an electrical field of approximately $500 \mathrm{~V} / \mathrm{in}, 80$ and reportedly emitted much more light than a lamp filled with air at low pressure.

The modified flashlamp is shown in Fig 24. The electrodes for the argon filled lamps were attached to the quartz tubing through 


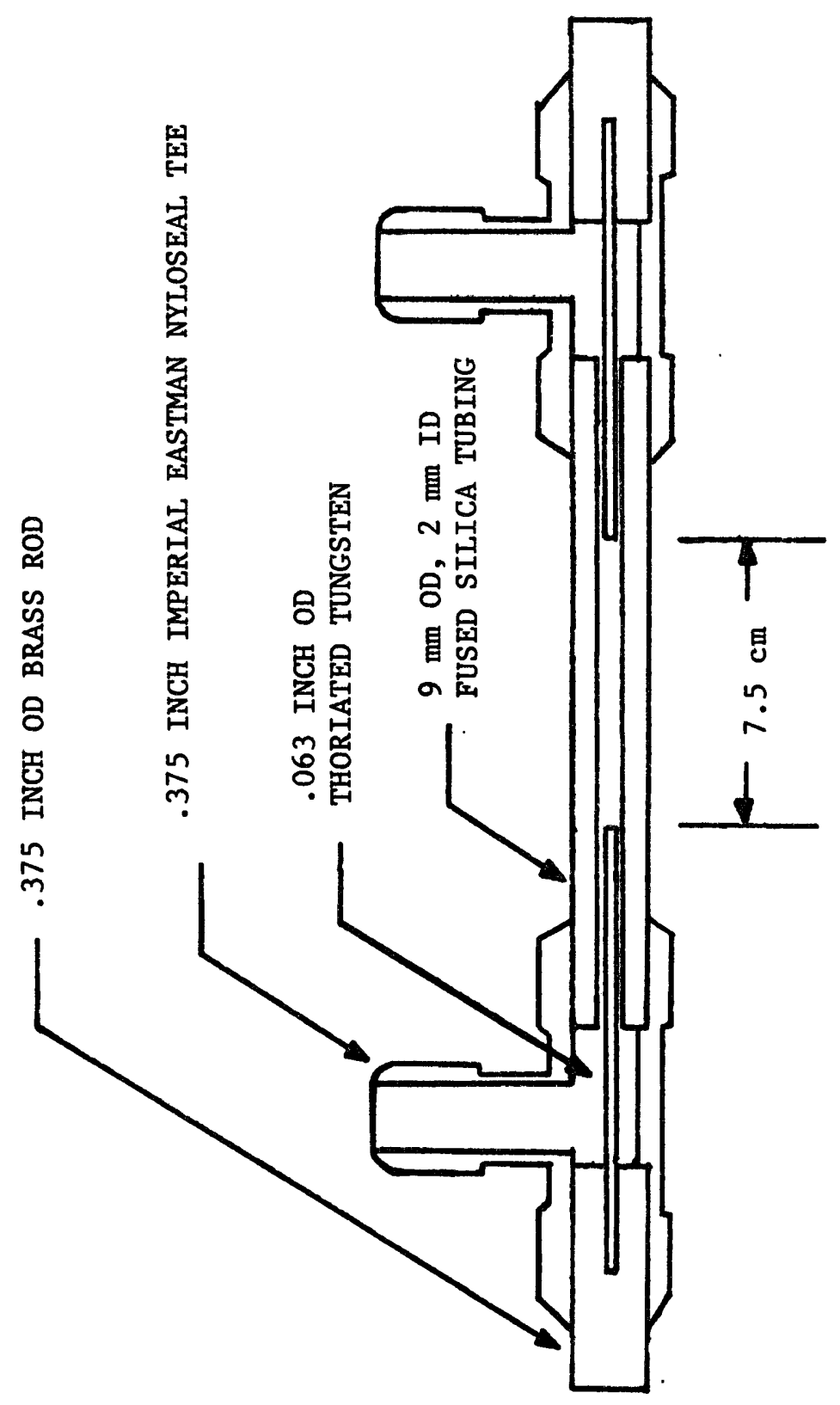


a $3 / 8$ inch Nyloseal tee connector. This method eliminated the troublesome epoxy seals. In addition the side inlet in the tee provided a convenient port for introduction and exhaust of gas. The flashlamp was constructed from pieces of $9.5 \mathrm{~mm}$ oD fused silica capillary tubing with $2 \mathrm{~mm}$ bore. The electrodes were pieces of .063 in. diameter, thoriated tungsten welding rod which were silver soldered into $.375 \mathrm{in.}$ OD brass rod. The tungsten rod extended into the quartz tubing bore, leaving 3 inches of arc length. These argon filled flashlamps were capable of three hours of continuous operation at $25 \mathrm{~J}$ input and $1 \mathrm{~Hz}$. After roughly 10,000 shots, deposits of reduced silicon would block the narrow annular gap between the tungsten electrode and the fused silica tubing thus stopping the flow of fresh argon. A distinctly visible enlargement due to erosion of the bore by the hot plasma could also be seen at this point. Micrometer measurements indicated a radial enlargement of roughly .020 inch after $\sim 10,000$ shots. While visible damage other than the ablated wall could not be seen, it is assumed that small cracks were developing in the flashlamp walls, since the flashlamps were subject to explosive failure after $\sim 18,000$ shots with $25 \mathrm{~J}$ input.

Despite the aforementioned problems, the argon flashlamps were the most satisfactory in that they were capable of three to five hours of operation at $1 \mathrm{~Hz}$ and $25 \mathrm{~J}$ and provided at least limited operation at full bank energy $(\sim 75 \mathrm{~J})$, whereas the earlier air-filled lamps did not. Moreover, they produced a significant $(20 \%)$ reduction in the amount of energy necessary for the dye laser to reach threshhold, 
and the dye laser light-out to energy-in efficiency approached 0.1 percent, a value close to that achieved by dye lasers cited in the literature. It should be noted that any changes which can be made which increase the efficiency of the dye laser will produce a drastic increase in flashlamp lifetime for the same laser output energy, sinee flashlamp lifetime $N$ (in shots) is related to lamp input energy (in Joules) by : 87

$$
N=\left(\frac{E_{x}}{E}\right)^{8.5}
$$

where $E_{x}$ is the single shot explosion energy (in Joules).

It was suggested that an additional increase in the efficiency of the flashlamp could be achieved if the fill pressure of the lamp was reduced from roughly 1 atmosphere to $1 / 2$ to $1 / 3$ atmosphere. Tests were conducted to determine the validity of this suggestion. Over the pressure range of 0.1 atmosphere to 1 atmosphere, no increase in efficiency was found. For this reason the lamps were operated with flowing argon gas at approximately 1 atmosphere. Operation at higher pressures was not attempted because pressurization would have required extensive modification of the flashlamps.

Measurement of the flashlamp current was attempted both using a current viewing resister placed in the flashlamp's ground return lead and with a current loop placed near the flashlamp leads. Both methods failed because the large amount of RFI generated by the spark gap rendered the oscilloscope trace unusable.

The light emitted by the flashlamp was monitored using a United Detector model PIN 10D silicon photodiode having better than $10 \mathrm{nS}$ 
response, and a Tektronix 7603 oscilloscope. The lamp output is shown in Fig. 25. As can be seen, the flashlamp pulse length departs considerably from the estimated $0.8 \mu \mathrm{s}$, suggesting that the inductance was underestimated.

Having described the construction and operation of the flashlamp pumping system, the next major section will deal with the design and construction of the dye cell, the optical component which couples the light from the flashlamp into the dye medium.

\section{Design of a Dye Cell and Dye Circulation System}

In this section both the optical system needed to couple the light from the flashlamp into the dye cell and the pumping system used to circulate the dye solution through the dye cell will be described.

Dye Ce11 Design and Construction: The flashlamp and dye cell are optically coupled by placing them at opposite foci of an elliptical cavity. In this way light emitted by the flashlamp is focussed by the elliptical surface onto the dye cell, as shown in Fig. 26. The position of the lamp and dye cell within the elliptical cavity can be determined from the equation of an ellipse. The foci separation distance $s$ is given by: 88

$$
s=2 \sqrt{a^{2}-b^{2}}
$$

where

$$
\begin{aligned}
& a=\text { the major axis radius } \\
& b=\text { the minor axis radius }
\end{aligned}
$$

The minimum value of $s$ is dictated by mechanical considerations since the dye cell and the flashlamp cannot occupy the same space, and 


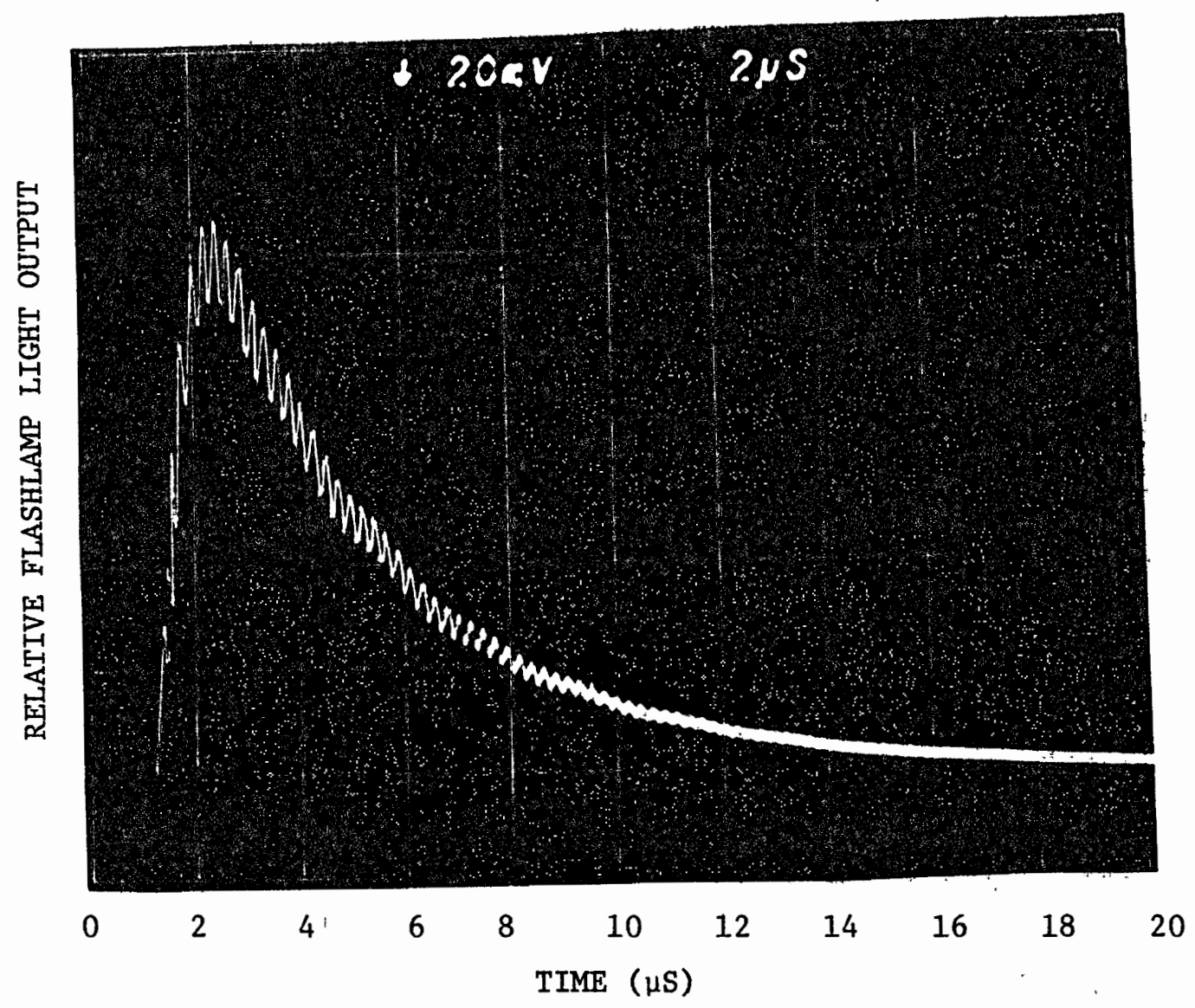

Fig. 25. Flashlamp 11ght output as a function of time. 


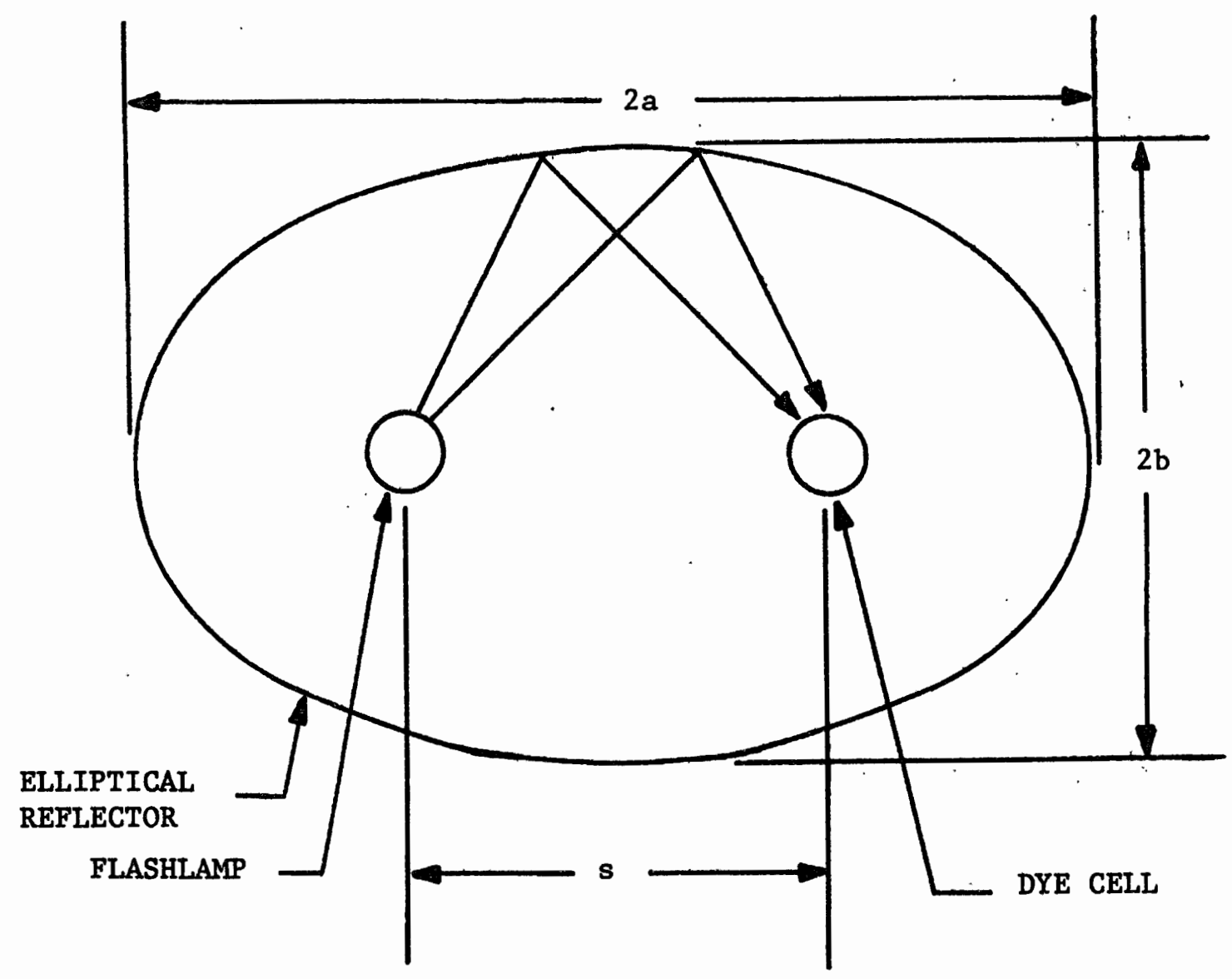

Fig. 26. Cross section of elliptical dye cell cavity. 
additional separation must be provided to allow for windows, seals, etc.

The length of the dye cell, and therefore of the elliptical cavity, was chosen to be three inches. This length was chosen on the basis of what appeared to be in use by those publishing in the literature. As it was initially decided to use $7.5 \mathrm{~mm}$ OD fused silica tubing with $1 \mathrm{~mm}$ walls for both the dye cell and the flashlamp, mechanical considerations suggested a minimum center to center spacing of $.490 \mathrm{in.}$ between the dye cell and the flashlamp. It was also decided an ellipse roughly two inches in diameter would constitute a reasonable choice for the size of the ellipse. The reason for the choice of such a large ellipse was that the degree of ellipticity would be small and thus provide more uniform excitation of the dye cel1. $^{88}$ Moreover, since the degree of ellipticity is small, the ellipse could be fabricated by deforming a two in. diameter circular tube.

The dimensions of an ellipse formed by deforming a circular tube can be determined as follows. The area of the circular tube is:

$$
A_{c}=\pi r^{2}
$$

Conversely, the area of the ellipse is given by:

$$
A_{e}=\pi a b
$$

Since slightly deforming the tube does not appreciably affect the area of the tube, the two areas must be roughly equal

$$
A_{c} \simeq A_{e} \text {. }
$$

Hence:

$$
b=\frac{r^{2}}{a}
$$


Substituting $(6.10)$ into $(6.6)$ one gets:

$$
s=2\left[a^{2}-\frac{r^{4}}{a^{2}}\right]^{1 / 2}
$$

Solving for $a$,

$$
a=\left[\frac{s^{2}}{8}+\left(\frac{s^{4}}{64}+r^{4}\right)^{1 / 2}\right]^{1 / 2}
$$

Using a two in. diameter tube to produce an ellipse with foci spaced .490 in. apart, the computed values for $a$ and $b$ are 1.015 and .985 inches respectively. As can be seen, very little deformation of the tube was necessary to produce an ellipse of the required dimensions. The ellipse was formed from a circular brass tube which was turned to an ID of 2.000 in. The circular tube was then deformed in a vice until it had the proper dimensions, and brass plates with elliptical holes to match the shape of the now elliptical tube were silver soldered to the ellipse to prevent it from relaxing to its previous circular shape and also to provide a surface perpendicular to the ellipse to which additional mechanical components could be mounted. The inside of the tube was polished first using very fine sandpaper, and then cerium oxide. The polished reflector was then given a reflective coating of evaporated aluminum.

Initially the laser was tested without the benefit of any coolant, however after a few seconds of operation at a PRF of $2 \mathrm{~Hz}$, the pulse-to-pulse stability of the laser declined dramatically, and the mean output power declined to a value roughly one quarter as large as that which was seen at low PRF's $(<.5 \mathrm{~Hz})$.

The reason for the unstable operation and reduced output power 
can be attributed to thermo-optic distortion due to heating of the dye solution. This hypothesis was checked by passing the beam from a helium neon laser through the dye solution. Without the flashlamp operating, the transmitted helium neon beam was both spatially and tempora1ly stable. When the flashlamp was started, however, the helium neon beam expanded from its normal area of a few square millimeters, to a spot of roughly one square centimeter in area when viewed on a screen roughly one-half meter from the dye cell. In addition, the probe beam was no longer temporally stable, but took on a boiling appearance. For this reason, it was felt that it would be necessary to cool the flashlamp if pulse-to-pulse stability was to be achieved at repetition rates of one pulse per second or more.

In addition; while the evaporated aluminum formed a highly reflective coating, it corroded almost immediately when cooling water was introduced into the cavity. In an attempt to solve the corrosion problem, a $12 \mathrm{~mm}$ OD flow tube was. fitted over the flashlamp. In this way cooling water could be forced around the flashlamp without contacting the aluminized reflector surface. This method of cooling, however, was detrimental to the operation of the dye laser, and the output energy from the laser was very erratic as compared to that obtained when no coolant was used and the laser was operated at a low PRF such that little thermo-optic distortion took place. It was decided to reattempt to cool the laser by flooding the elliptical cavity. To eliminate the corrosion problems present with the aluminized ellipse, the ellipse was repolished and plated with nickel. The nickel surface has a lower theoretical reflectivity than the aluminized 
one (64 percent for nickel as opposed to 92 percent for aluminum at $546 \mathrm{~nm}^{89}$ ), but its corrosion resistance is far ouperior. While the nickel did not corrode, the laser still required a large amount of energy $(\sim 20$ Joules $)$ into the flashlamp to reach threshold. Similar dye lasers, such as the one marketed by Chromatix, have a threshold input energy of roughly 5 Joules. Following conversations with Dr. G. A. Massey of the Oregon Graduate Center, it was decided that the fault might lie in the design of the elliptical reflector itself. The reason is that much of the flashlamp light is lost due to scattering from the unpolished end plates. Following his suggestion a simple reflector was constructed by closely wrapping aluminum foil around the flashlamp and dye cell: The resultant reflector, while not elliptical, does have a much larger length-to-width aspect ratio, thus reducing the loss to the ends of the dye cavity. The resultant combination of the aluminum foil reflector and argon filled flashlamp yielded a dye laser which would threshold at 6 Joules untuned, which is comparable to thresholds found in commercial flashlamp pumped dye lasers. Moreover, the dye laser light output energy to flashlamp input energy approached 0.1 percent, a value close to that achieved in similar dye lasers cited in the literature.

The dye laser output energy was measured using a calibrated integrating silicon detector borrowed from the Oregon Graduate Center. A plot of the untuned dye laser output energy versus input electricál energy to the flashlamp is shown in Fig. 27.

Dye Mixture and Its Circulation System: In a flashlamp pumped 


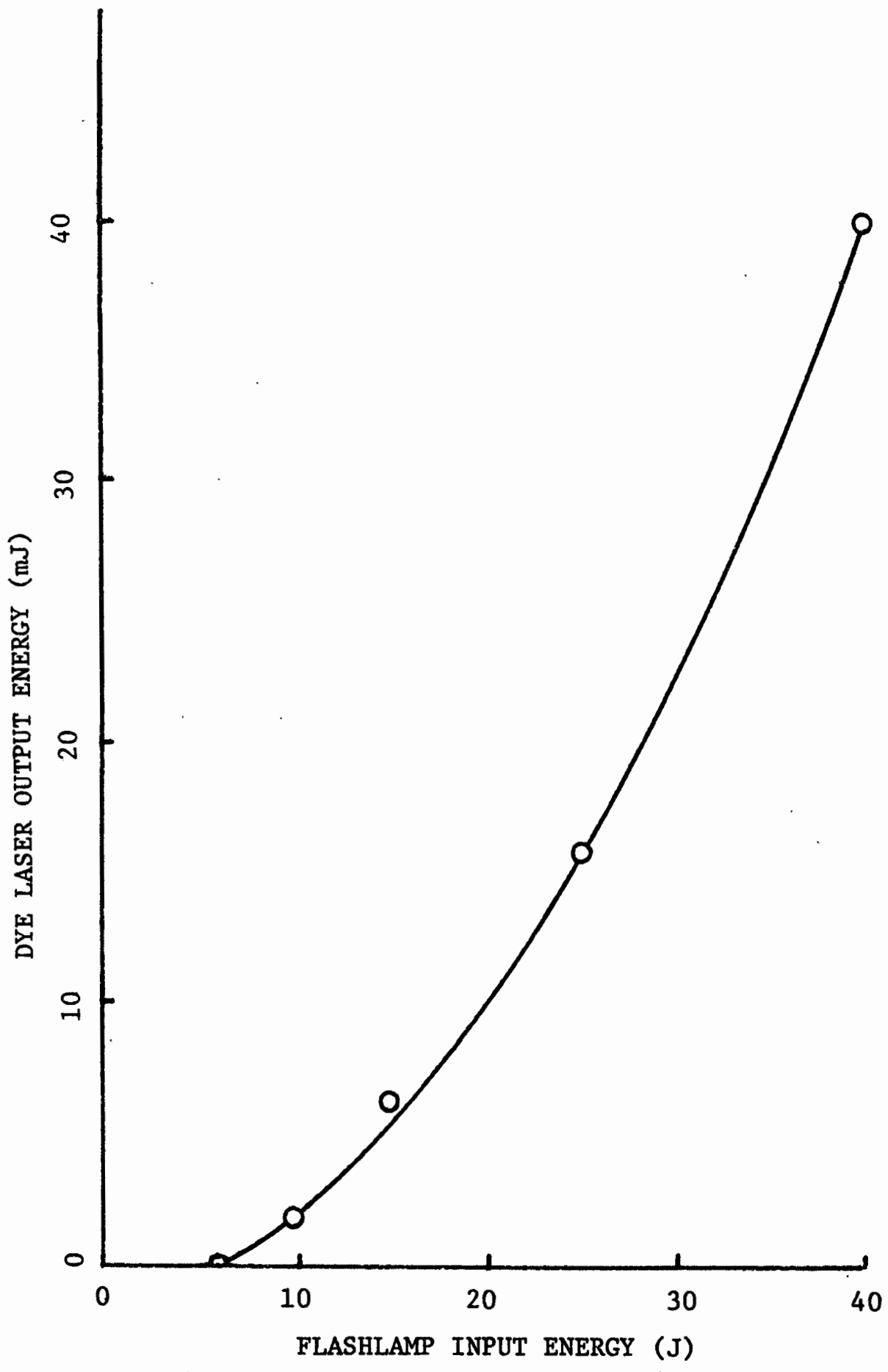

Fig. 27. Broadband dye laser output as a function of electrical Input energy. 
dye laser, much of the flashlamp light which is absorbed by the dye solution is converted to heat, thus raising the temperature of the dye solution. The warmed dye will begin to cool by conduction through the wall of the dye flowtube creating a nonuniform temperature distribution within the dye flowtube. The effect of the nonuniform temperature distribution upon a laser beam passing through the heated dye is the same as that of atmospheric turbulence except that the magnitude of the beam distortion for a simflar pathlength will be orders of magnitude worse. In fact, heated tanks of alcohol, a common solvent for laser dyes, are currently used to model the effects of atmospheric turbulence upon laser beam propagation since a tank of warm alcohol just a few feet long can simulate an atmospheric path many kilometers long. To minimize the index of refraction gradients in the dye solution, pure alcohol is seldom used in flashlamp pumped systems, but rather a mixture of alcohol and water. It is easy to show that the change in the index of refraction of a material $\Delta n$, due to the absorption of an amount of energy $E$ is given by:

$$
\begin{aligned}
\Delta \mathrm{n} & =\left(\frac{\mathrm{dn}}{\mathrm{dT}}\right) \Delta \mathrm{T} \\
& =\frac{\mathrm{E}}{\mathrm{c}_{\mathrm{p}} \rho}\left(\frac{\mathrm{dn}}{\mathrm{dT}}\right)
\end{aligned}
$$

where $c_{p}$ is the specific heat of the material and $p$ is its density. To evaluate the relative merit of using water as opposed to a typical alcohol such ethanol consider the ratio of the $\Delta n^{\prime} s$ :

$$
\left(\frac{\Delta n_{W}}{\Delta n_{A}}\right)=\left(\frac{c_{p A} P_{A}}{c_{p W} P_{W}}\right) \frac{\left(\frac{d n}{d T}\right)_{W}}{\left(\frac{d n}{d T}\right)_{A}} .
$$


Taking as values for the constants: $c_{p A}=2.4 \mathrm{~J} / \mathrm{gr} / \mathrm{C}, \mathrm{c}_{\mathrm{pW}}=4.2 \mathrm{~J} / \mathrm{gr} / \mathrm{C}$, $\rho_{\mathrm{A}}=.8 \mathrm{gr} / \mathrm{cm}^{3}, \rho_{\mathrm{W}}=1.0 \mathrm{gr} / \mathrm{cm}^{3},\left(\frac{\mathrm{dn}}{\mathrm{dT}}\right)_{\mathrm{A}}=4 \times 10^{-4},\left(\frac{\mathrm{dn}}{\mathrm{dT}}\right)_{\mathrm{W}}=1 \times 10^{-4}, 92$ it can be seen that for equal volumes of the two solvents, the index shift of the water will be 8 times smaller than that of pure alcohol. The index shift of a solution of the two will be somewhere in between the index shifts of the two pure solvents and inversely proportional to the quantity of each liquid making up the solution. The dye solution used in the dye laser was roughly $10^{-4}$ molar in a 3:1 water:ethanol solution. The use of pure water is precluded by the fact that the efficiency of rhodamine dyes in pure water is much less than when alcohol is present. ${ }^{73}$

While the use of water as a solvent does reduce the problem of refractive index gradients, the reduction is not generally sufficient to allow laser operation at repetition rates of $1 \mathrm{~Hz}$ without replacing the dye between laser firings. For this reason dye lasers usually employ a pump to circulate dye from a reservoir through the dye laser and back to the reservoir. To avoid the problems of dye contamination by lubricants present in pumps which have rotary sea1s, most dye lasers use pumps whose gears or impellers are separated from the motor by a thin non-magnetic material which usually forms one wall of the pump. Rotary motion between the motor and the pump is coupled through the thin non-magnetic wall of the pump by a pair of magnets. A magnetically coupled gear pump was purchased from Micropump and was used to circulate the dye. The dye was normally circulated at a flow rate of approximately one liter per minute.

Many papers in the literature indicate the use of filters 
between the pump and the dye cell to remove small air bubbles which would cause a scattering loss for the dye laser. A filter was therefore constructed for use with the dye laser. It was soon found to be unnecessary since the major source of bubbles was cavitation within the dye cell. The filter was therefore not used.

\section{Design of the Laser Resonator}

The resonator of any laser typically consists of two reflectors, one which reflects virtually all of the laser's light, and a second which is only partially reflecting and through which the output is taken. The optimum output coupler in terms of getting the maximum power out of a laser can be shown to be one whose transmission $T$ is given by: 90

$$
T=L+\sqrt{g L}
$$

where

$$
\begin{aligned}
& \mathrm{L}=\text { the unavoidable per pass loss within the laser resonator } \\
& \text { and includes such things as reflection losses from windows } \\
& \mathrm{g}=\text { the per pass gain of the laser. }
\end{aligned}
$$

If the gain is small as it will be in a dye laser which is tuned to either the long or short wavelength end of the dye's lasing range, then the optimum output coupler is one whose transmission is equal to the internal losses of the resonator. The diffraction grating was assumed to have 15 percent ${ }^{91}$ loss and the two windows on the ends of the dye cell were assumed to have reflection losses of 4 percent each. The total cavity losses were therefore estimated to be on the order of 23 percent. This would indicate that the 
optimum output coupler would have a reflectivity of 77 percent. Output coupler reflectivities found in the literature 92,93 indicated the reflectivities of 65 percent were more commonly used. It was decided to trust the experience of others and an output coupler having a reflectivity of 65 percent was purchased. It consisted of a multilayer dielectric coating which was applied to a 1 inch diameter by .250 inch thick fused silica substrate. The reflecting surface of the mirror was concave with a 10 meter radius of curvature. The curved surface is necessary for stable operation of the laser.

In most lasers, the second mirror in a laser is just that, a mirror; however, to exploit the wavelength tunability feature of the dye laser, some form of wavelength dispersive tuning element must be inserted into the dye laser cavity. Two such elements, a diffraction grating (Fig. 28) and flint glass prisms, (Fig. 29), were used in the dye laser.

Dye Laser Operation Using a Diffraction Grating as a Tuning Element:

The diffraction grating was an aluminum coated replica grating with 1200 grooves per millimeter and blazed at $500 \mathrm{~nm}$ was purchased from PTR Optics. The grating was used in a first-order Littrow configuration. In this mode, light which strikes the grating at an angle $\theta$ is reflected back on itself. The angle is given by: ${ }^{91}$

$$
\theta=\sin ^{-1}\left[\frac{\lambda}{2 d}\right]
$$

where

$$
\begin{aligned}
& \lambda=\text { the wavelength } \\
& d=\text { the groove spacing of the grating }
\end{aligned}
$$

The theoretical linewidth of a diffraction limited dye laser 


\section{DIFFRACTION}

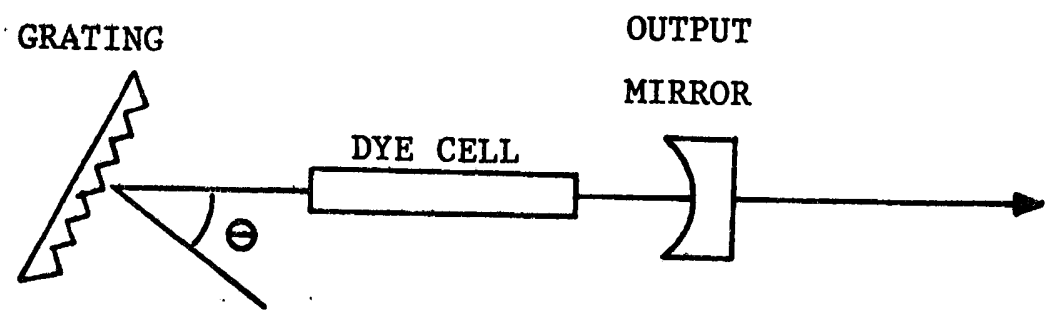

Fig. 28.
diffraction grating. 
TOTALLY REFLECTING

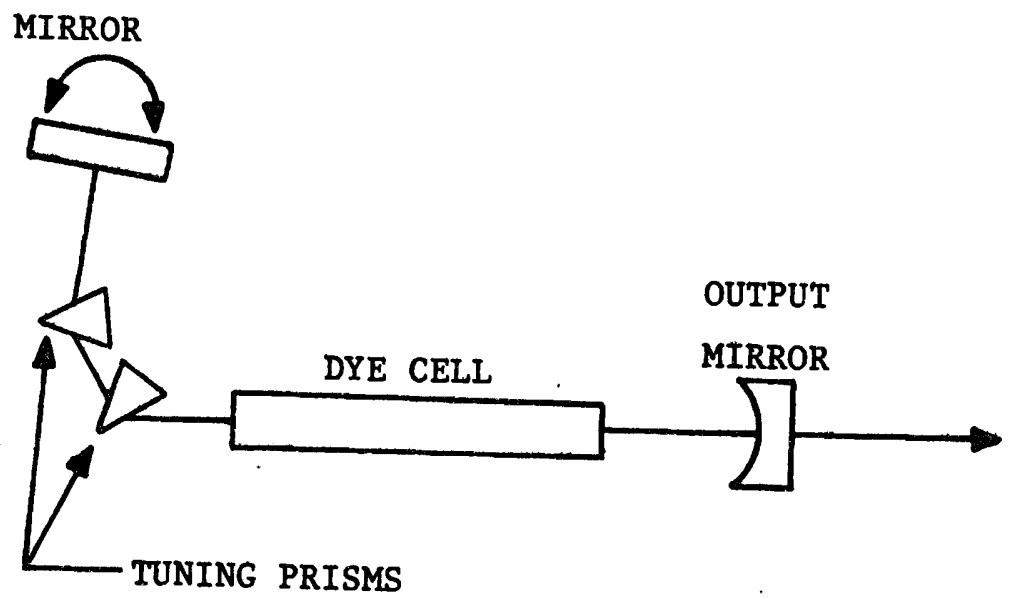

$\frac{\text { Fig. 29. }}{\text { elements. }}$ Dye laser configuration using two prisms as tuning 
using a grating as a dispersive element can be calculated from the following considerations. The angular resolution of any diffraction limited optical element was found by Lord Rayleigh to be: ${ }^{7}$

$$
\Delta \theta=\frac{1.22 \lambda}{D}
$$

where $\Delta \theta$ is the angular resolution of the optical system, $\lambda$ is the wavelength, and $D$ is the diameter of the limiting aperature (the beam waist diameter in the case of a laser beam). For a Littrow mounted diffraction grating the angular dependance of the first order diffracted beam as a function of wavelength was given to be:

$$
\theta=\sin ^{-1}\left[\frac{\lambda}{2 d}\right]
$$

Differentiating (6.16) one gets:

$$
\Delta \theta=\frac{1}{2 \mathrm{~d}} \frac{1}{\left[1-\left(\frac{\lambda}{2 \mathrm{~d}}\right)^{2}\right]^{1 / 2}} \Delta \lambda .
$$

On equating the two expressions for $\Delta \theta,(6.18)$ and (6.19) and solving for the wavelength spread

$$
\Delta \lambda=\frac{2.44 \lambda d}{D}\left[1-\left(\frac{\lambda}{2 d}\right)^{2}\right]^{1 / 2}
$$

The diameter of a diffraction limited beam waist in a laser cavity such as was actually used is given approximately by: 90

$$
D \simeq 2\left(\frac{\lambda l}{\pi}\right)^{1 / 2}\left[\frac{R^{2}}{\ell(2 R-\ell)}\right]^{1 / 4}
$$

Using as parameters typical of those found in our dye laser: a wavelength of $600 \mathrm{~nm}$, a cavity length of .5 m, and a mirror curvature of $10 \mathrm{~m}$, then the beam diameter is approximately $1.1 \mathrm{~mm}$. 
Using this as the beam diameter in (6.20) one finds the theoretical resolution of the PTR grating to be $\Delta \lambda=.88 \mathrm{~nm}$. The actual grating narrowed laser 1inewidth was measured using a hand held Fabry Perot etalon having a $1 \mathrm{~nm}$ free spectral range, and a finesse of 20 , to be on the order of $0.5 \mathrm{~nm} \pm 0.1 \mathrm{~nm}$, a value which is in fair agreement with what was expected from the analysis.

When the laser is operated using a grating as a tuning element and a polarized output is required for future beam use such as harmonic generation, it is sometimes necessary to introduce a polarizing element into the cavity. Since the grating used displays little preference with regard to polarization at rhodamine wavelengths, a stacked plate polarizer was constructed to ensure that the laser output was linear'y polarized. The polarizer was made of two glass windows placed at Brewster's angle such that" a beam polarized vertically would experience little loss, whereas the horizontally polarized beam would experience a loss per pass given by: 94

$$
\text { Loss/pass }=1 .-(.85)^{2 n}
$$

where

$$
\mathrm{n}=\text { the number of plates }
$$

For a two plate polarizer, the horizontally polarized component of the beam would experience a 108 s of $48 \%$ per pass. The polarizer was placed in the cavity between the dye cell and the output coupling mirror and produced a beam which was almost completely vertically polarized.

While the diffraction grating did produce a relatively narrow 
linewidth output, it was also inefficient in terms of reflecting laser light which was incident upon it, and therefore represented a substantial loss within the laser cavity. The dye laser produced very little output power and exhibited a very high threshold compared to its untuned configuration when the grating was used. An alternate and less lossy tuning method is the use of equilateral prisms, provided they can supply the necessary line narrowing. In the next section, the use of tuning prisms and their performance relative to that of the diffraction grating will be described.

\section{Dye Laser Operation Using Flint Prisms as Tuning Elements:}

As in the case of the diffraction grating, it should be possible to predict the linewidth of the prism tuned dye laser output by following the same procedure as was used with the diffraction grating, i.e. by computing the angular dispersion of the tuning element, in this case the prism, and equating that to the diffraction angle of the dye laser beam. We will assume that the prism is used at minimum deviation, as shown in Fig. 30. When used at minimum deviation, the angle $\phi$ formed by the entering beam and the entrance surface will be the same as the respective angle $\phi^{\prime}$ formed by the emerging beam and the emergent surface. Moreover, the entering and exiting beams will have to first order the same diameter. The index of refraction $n$ of a prism used in this manner is given to be a function of the angle of deviation $\theta$ and the prism apex angle $\alpha$, as follows: ${ }^{7}$

$$
n=\frac{\sin \frac{1}{2}(\theta+\alpha)}{\sin \frac{\alpha}{2}} .
$$




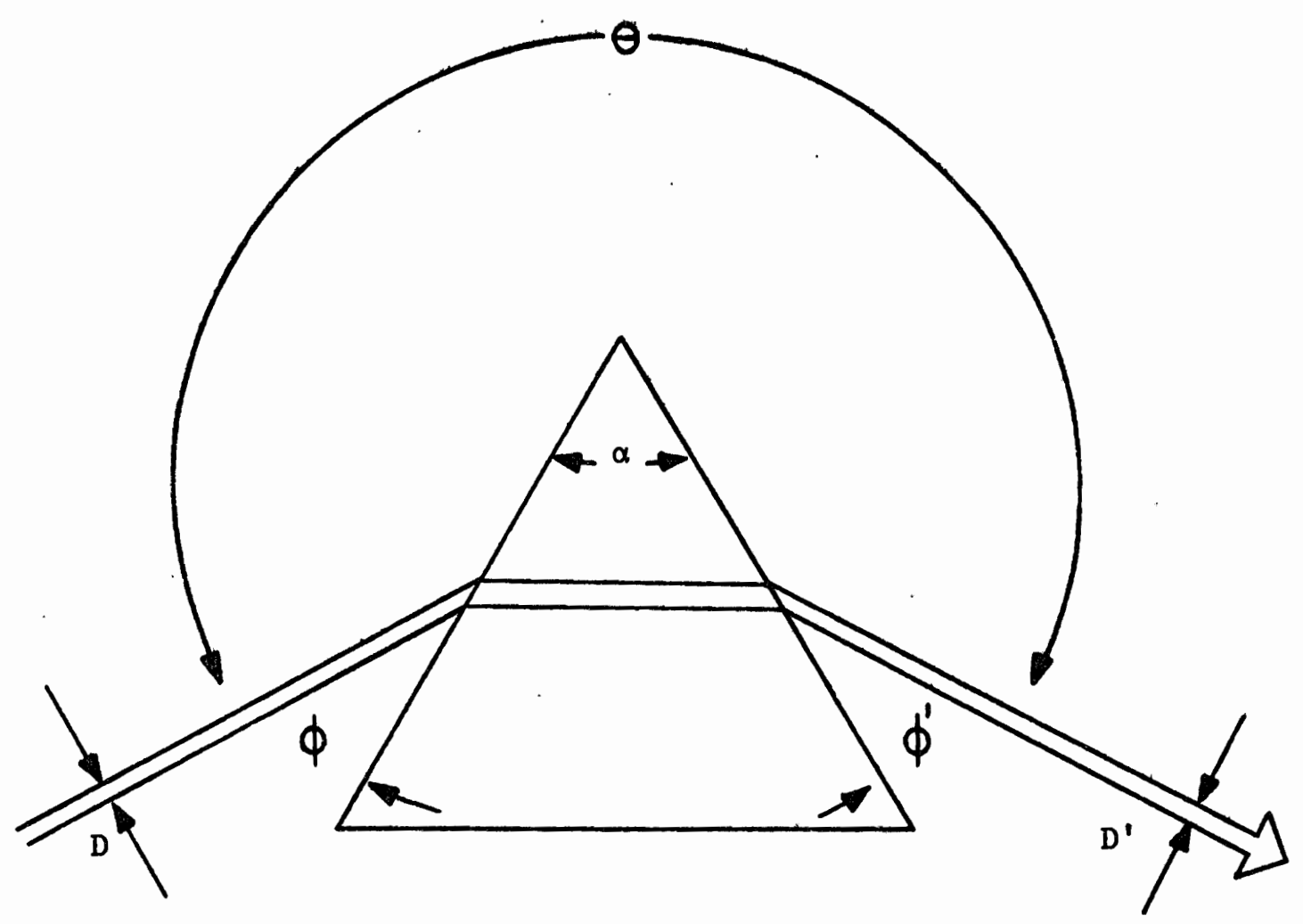

Fig. 30. Tuning prism showing minimum deviation condition, $\phi=\phi^{\prime}$. 
The parameter of interest in determining the resolution of the prism, is the amount of angular dispersion per unit of wavelength, since this quantity can be related to the diffraction limited acceptance angle (equation (6.18)) just as it was in the case of the diffration grating. Defining a new variable $u$ to be

$$
u \equiv \frac{\theta+a}{2}
$$

and through use of the chain rule, it is possible to solve for the angular dispersion of the prism $\frac{d \theta}{d \lambda}$ :

$$
\begin{aligned}
\frac{d \theta}{d \lambda} & =\frac{d \theta}{d u} \frac{d u}{d n} \frac{d n}{d \lambda} \\
& =\frac{2 \sin \left(\frac{\alpha}{2}\right)}{\left[1-n^{2} \sin ^{2}\left(\frac{\alpha}{2}\right)\right]^{1 / 2}} \frac{d n}{d \lambda} .
\end{aligned}
$$

where $\frac{d n}{d \lambda}$ is recognized to be the spectral dispersion of the prism material. The wavelength spread $\Delta \lambda$ for a dispersed spectra of angular width $\Delta \dot{\theta}$ is then given by

$$
\Delta \lambda=\frac{\left[1-n^{2} \sin ^{2}\left(\frac{\alpha}{2}\right)\right]^{1 / 2}}{2 \sin \left(\frac{\alpha}{2}\right) \frac{d n}{d \lambda}} \Delta \theta
$$

But $\Delta \theta$ for a diffraction limited beam was given by (6.18):

$$
\Delta \theta=\frac{1.22 \lambda}{\mathrm{D}} \text {. }
$$

Inserting (6.18) into (6.27) we get an expression for the wavelength spread of a diffraction limited beam of diameter $D$, which can be expected from a laser using an intracavity prism as a tuning element. That expression is: 


$$
\Delta \lambda=\frac{.61 \lambda\left[1-\mathrm{n}^{2} \sin ^{2}\left(\frac{\alpha}{2}\right)\right]^{1 / 2}}{D \sin \left(\frac{\alpha}{2}\right) \frac{\mathrm{dn}}{\mathrm{d} \lambda}}
$$

The prisms used in our dye laser were equilateral spectroscope prisms constructed from SF-2 glass. The index of refraction and dispersion for this glass in the spectral region of interest is: ${ }^{95}$

$$
\begin{aligned}
\mathrm{n} & =1.6490 \\
\frac{\mathrm{dn}}{\mathrm{d} \lambda} & =8.35 \times 10^{-5} \mathrm{~nm}^{-1}
\end{aligned}
$$

Substituting these values into the wavelength spread equation (6.28) together with the appropriate dye laser parameters, $\lambda=600 \mathrm{~nm}$, and $D=1.1 \mathrm{~mm}$, we get as an expected wavelength spread of $4.5 \mathrm{~nm}$.

Two of the equilateral tuning prisms were available, and since, the dye laser linewidth should be inversely proportional to the number of prisms used, both were used. The linewidth was again measured using the sma11, hand held Fabry Perot etalon described in the previous section, and was again found to be on the order of $0.5 \mathrm{~nm}$. This is much narrower than the analysis would have predicted.

Using the prisms as tuning elements, the dye laser operated in a much more stable fashion (less pulse-to-pulse power fluctuations, lower threshold, etc.) than with the diffraction grating in the cavity. For this reason, it was decided to use the tuning prisms as the line-narrowing elements instead of the grating.

When tuning prisms are used, it is usually not necessary to provide an additional polarizer, since the prisms only display low loss for the polarization component of the light beam lying in the plane of the paper in Fig. 30, the $P$ component.

Some attempt was made to increase the line narrowing capacity 
of the tuning elements by increasing the diameter of the beam through the introduction of a $\times 10$ intracavity telescope. However, due to the lack of low-loss anti-reflection coated lenses of diffraction limited quality; the output energy of the dye laser dropped. Had high quality lenses been available, the linewidth could likely have been reduced by a factor of perhaps ten. Such a decrease in laser linewidth would increase the efficiency of the frequency doubling process as will be discussed in Chapter VII.

Methods for Producing Simultaneous or Near Simultaneous Outputs At Two Wavelengths

In Chapter II it. was suggested that one method for reducing the problems of atmospheric turbulence in making a dual wavelength measurement would be to perform the two measurements within approximately 16 microseconds of each other, so as to "freeze" the turbulence. In this section two possible schemes will be discussed.

The first scheme (Fig. 31) involves using two diffraction gratings together with a polarizer to form two independently tunable dye laser cavities, which share a common active medium. ${ }^{96}$ This method has been tested in the laboratory, and has one real problem. When the two wavelengths are tuned close to one another, the dye laser will preferen-. tially lase at the wavelength for which the gain/loss ratio is highest. Since the gain curve for a dye is a convex function, it is necessary to tune the losses of the two branches until the gain/loss ratios are the same. If the dye laser is: to be tuned to a number of different pairs of wavelengths, then the loss will have to be adjusted for each 


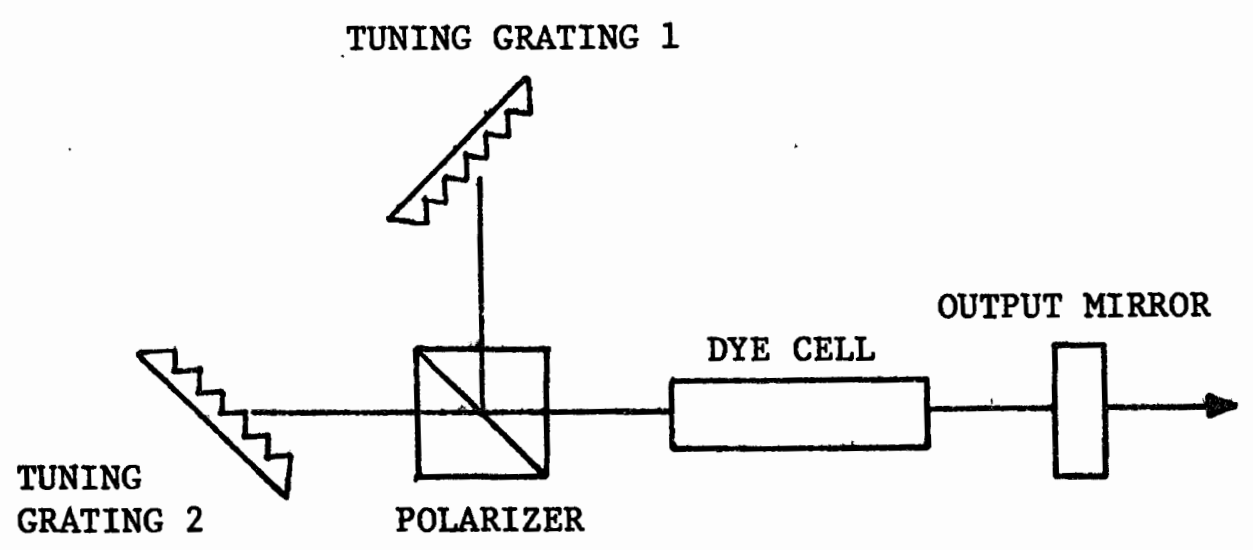

Fig. 31. Dye laser tuning method capable of producing simultaneous outputs at two different wavelengths. 
wavelength pair. While it would be useful to have a laser which would simultaneously emit two wavelengths, the necessary additional complexity of having to have a controlled, variable loss inside the laser cavity more than outweighs the benefits accrued.

Since it is only necessary to produce the two wavelengths within a period of 16 microseconds of each other, one could think about physically rotating the tuning grating by an amount necessary to tune the dye laser to the second wavelength in the wavelength pair. As anyone who has attempted to construct a device to rotate a physical object such as a diffraction grating by a very small but precise amount in a very short period of time will testify, such a task is difficult. A more elegant method of accomplishing the same end is to rotate the beam relative to the grating rather than the grating (see Fig. 32). While a wide variety of beam deflection methods exist, only one example need be given to demonstrate the attractiveness of this scheme.

For a diffraction grating, the required deflection angle $\theta$, necessary to tune between two wavelengths separated by $\Delta \lambda$ can be shown to be: ${ }^{91}$

$$
\Delta \theta=\frac{2 \Delta \lambda}{\left(4 d^{2}-\lambda_{B}{ }^{2}\right)^{1 / 2}}
$$

where

$$
\begin{aligned}
& d=\text { the groove spacing of the grating } \\
& \lambda_{B}=\text { the blaze wavelength of the grating }
\end{aligned}
$$

Also, for an electro-optic beam deflector of the type described by Beasely, ${ }^{97}$ and shown in Fig. 33, the deflection angle is given 


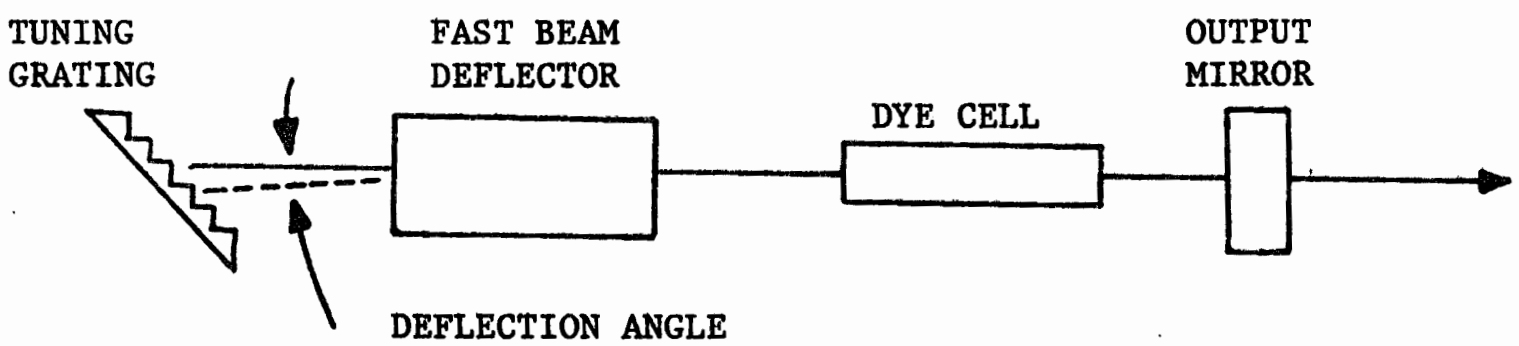

Fig. 32. Dye laser tuning scheme utilizing a fast beam deflector to tune the laser from one wavelength to another closely spaced wavelength. 


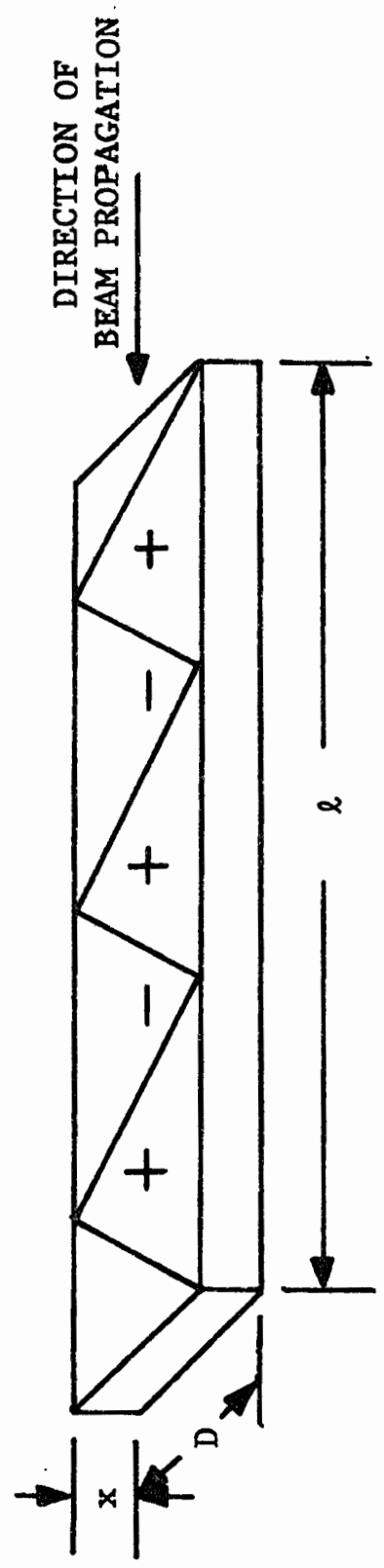

कू

D

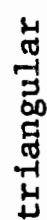

足

मै

논

d

प्त

क्त

है

م

ن

苟范

엉 -

요

出

บै

o.

号

त्ञ

岱

药

4 胥

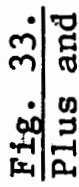


by:

$$
\Delta \theta=\frac{\ell n^{3} r V}{2 D x}
$$

where:

$$
\begin{aligned}
& \ell=\text { the length of the deflector crystal } \\
& \mathbf{n}=\text { the index of refraction of the deflector material } \\
& \mathbf{r}=\text { the appropriate electro-optic coefficient } \\
& \mathrm{V}=\text { the applied voltage } \\
& D=\text { the beam diameter } \\
& \mathrm{X}=\text { the crystal thickness }
\end{aligned}
$$

Equating (6.29) and (6.30) and solving for the necessary applied voltage, the resulting expression for the necessary applied voltage is :

$$
v=\frac{4 D x}{\left(4 d^{2}-\lambda_{B}{ }^{2}\right)^{1 / 2} \ln ^{3} r} \Delta \lambda
$$

Assuming as reasonable values for the parameters: $D=x=2, \mathrm{~mm}, \mathrm{~d}=8.33 \times 10^{-7} \mathrm{~m}, \lambda_{B}=5 \times 10^{-7} \mathrm{~m}, l=5 \mathrm{~cm}, \mathrm{n}^{3} \sim 3.5$, $r \sim 2 \times 10^{11} \mathrm{~m} / \mathrm{V}$ (a value typical for two electro-optic materials, $\mathrm{ADP}$ and $\mathrm{KD} * \mathrm{P}),{ }^{96}$ and a tuning range of $1 \mathrm{~nm}$ such as tuning between an $\mathrm{SO}_{2}$ peak and valley, we would have to apply a voltage of approximately 2900 volts. This could easily be done using a pair of high voltage transisters such as Sylvania's ECG - 164 .

In summary, there appears to be at least one reasonable method of rapidly tuning a dye laser between two different wavelengths within a time short enough to freeze the turbulence. 
CHAPTER VII

DESIGN AND CONSTRUCTION OF A DYE LASER FREQUENCY DOUBLER

In the preceding chapter, the design and construction of a flashlamp-pumped dye laser was described. Once the dye laser was constructed, it was necessary to shift the dye laser output into the ultraviolet region.

As has been mentioned in earlier sections, the method of producing ultraviolet light from visible light is through a process known as frequency doubling. 59 In this process visible light from a laser is incident on a crystalline material.

If the response of the crystal's electrons to the incident field is linear, then the electrons will have no influence upon the frequency of the light beam passing through the crystal. If the response is nonlinear, then the electrons will interact with the incident beam of frequency $\omega$ to a new, generated beam of frequency $2 \omega$. The formalism relating to this process is available in the literature, and will not be reproduced here except to quote the final result relating the second harmonic power $P_{2}$ to the first harmonic power $P_{1}: 59$

$$
\mathrm{P}_{2}=\frac{52.2 \mathrm{~d}^{2} \ell^{2} \mathrm{P}_{1}^{2}}{\mathrm{n}^{3} \lambda_{2}{ }^{2} \mathrm{~A}} \frac{\sin ^{2}\left(\frac{\Delta \mathrm{k} \ell}{2}\right)}{\left(\frac{\Delta \mathrm{k} \ell}{2}\right)^{2}}
$$


where:

$d=$ the nonlinear coefficient of the crystal

$\ell=$ the length of the crystal

$\lambda_{2}=$ the second harmonic wavelength

$\mathrm{n}=$ the average index of refraction of the crystal

$\Delta \mathrm{k}=$ the phase mismatch

$$
=2 \pi\left(\frac{2 n_{1}}{\lambda_{1}}-\frac{n_{2}}{\lambda_{2}}\right)
$$

$A=$ the area of the beam

As can be seen, the second harmonic power scales with the square of both the fundamental power and the crystal length. A severe restriction upon the efficiency of the SHG process results from the sinc function whigh has zeroes when $\frac{\Delta k \ell}{2}= \pm \pi$ and is equal to $1 / 2$ when $\frac{\Delta k \ell}{2}= \pm 1.392$. If $\frac{\Delta k l}{2}$ is equal to 0 , then the value of the sinc function is 1.0 , and maximum conversion efficiency occurs. When $\Delta k=0$, the process is known as perfectly phase matched.

The remainder of this chapter will deal with the limitations imposed upon the SHG process by the sinc function. The first topic to be discussed will be methods of achieving phase matching. Also included in the first section is a brief discussion related to the choice of a frequency doubling crystal. This topic is included at this point since the method of phase matching chosen will depend upon the particular crystal chosen. The method of phase matching which will be chosen is termed angle matching, and in the second section of the chapter some of the considerations relevent to this particular phase matching process will be discussed. The third section of the chapter 
will deal with the spectral bandwidth limitation of the frequency doubler, while the final section of the chapter will deal with the experimental details of cutting and polishing an ammonium dihydrogen phosphate (ADP) frequency foubling crystal.

\section{Methods of Phase Matching}

As was stated in the preceding section, perfect phase matching can only occur when the index of refraction of the crystal at the second harmonic frequency is equal to the index of refraction at the fundamental frequency. The physical significance of this relation is that for the second harmonic wave to grow, it must travel at the same phase velocity as the fundamental wave. This can only be true if the index of refraction seen by the generated second harmonic is the same index seen by the fundamental beam, a condition which cannot be met in most materials since the index of refraction of most optical mediums increase as one goes towards shorter wavelengths. For. crystalline materials which lack inversion symmetry, the index of refraction seen by a light beam will depend both upon the direction of propagation through the crystal as well as the polarization of the light beam relative to the crystalline axis. Crystals such as the one used in this investigation have two indices of refraction, one termed ordinary, and the second termed extraordinary, and such crystals are termed uniaxial. To determine the index of refraction as seen by an incident beam one needs to examine the projections of the incident E field upon the crystalline axis (see Fig. 34$)^{59}$. An incident beam whose electric vector lies in the $x-y$ plane $\left(e . g\right.$. $\left.E_{1}\right)$ will see the 


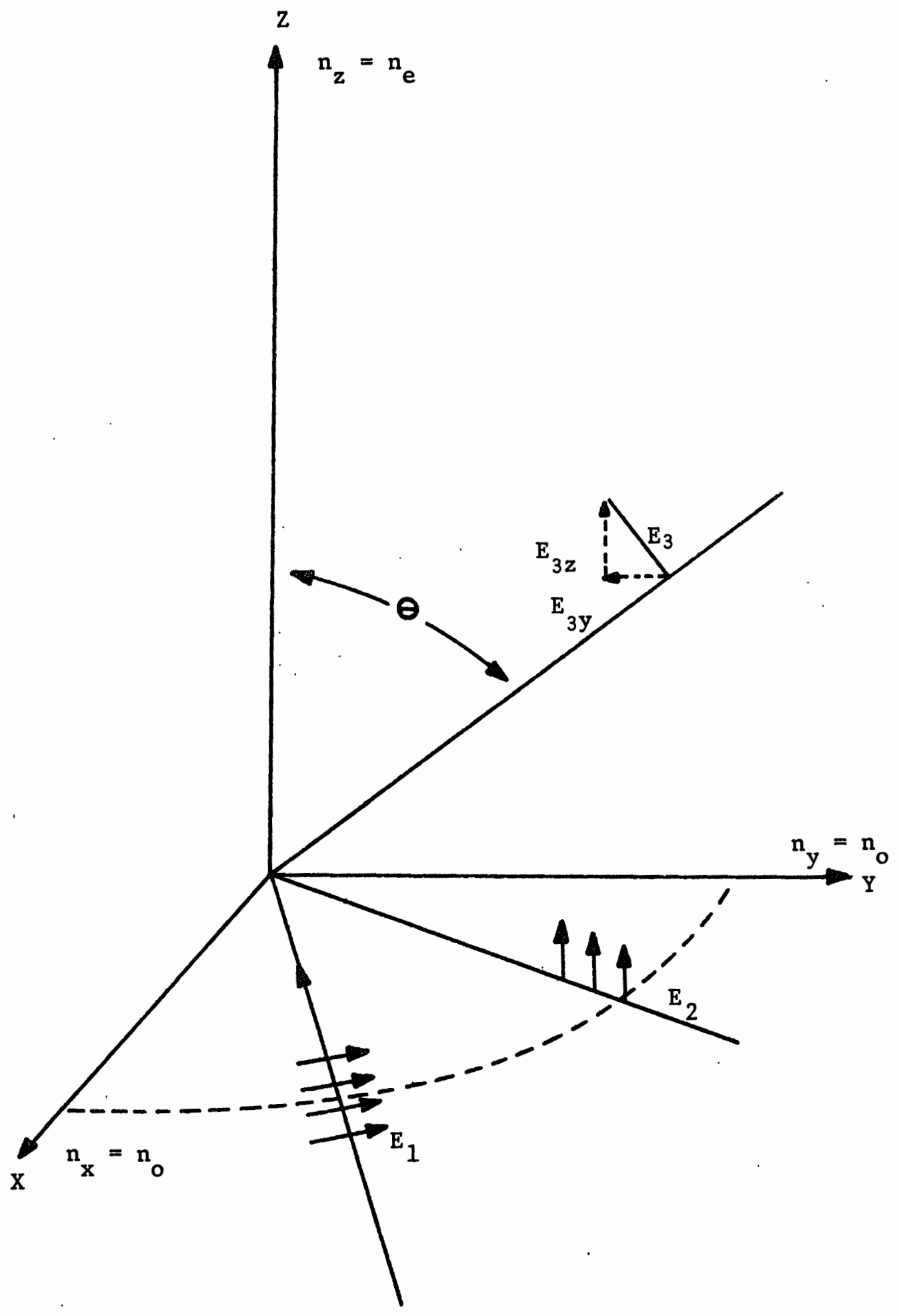

Fig. 34. Crystalline axis showing the indices seen by various incident electric fields. The crystal is taken to be uniaxial. 
crystal's ordinary index of refraction $n_{0} \cdot$ Beams which are incident: upon the crystal with their electric vectors perpendicular to the $x-y$ plane and therefore parallel to the crystal's $z$ axis (e.g. $E_{2}$ ) will see the crystal's extraordinary index $\mathrm{n}_{\mathrm{e}}$. The dispersion relations for a typical uniaxial crystal plotted in Fig. $35 .{ }^{98}$ As can be seen, it is conceivable that one could achieve phase matching by orienting the pump beam polarization in such a way that it sees the crystal's ordinary index, whereas the second harmonic sees the extraordinary index.

If the fundamental beam is propagated in the $x-y$ plane and has its polarization parallel to the $x-y$ plane, and the second harmonic has its polarization parallel to the $z$ axis, then the SHG process is known as $90^{\circ}$ phase matched. For any particular crystal and temperature, the shortest fundamental wavelength which can be perfectly phase matched is one which is $90^{\circ}$ phase matched. As an example, the $90^{\circ}$ phase matching wavelength for the crystal ADP at room temperature is $524 \mathrm{~nm}^{99}$ It is possible to increase the phase matched wavelength in most crystals by increasing their temperature, since the effect of increasing the crystal temperature is to move the extraordinary index curve closer to the ordinary index curve. ${ }^{100}$ The maximum temperature for crystals such as ADP is limited by thermal. decomposition of the crystal, and raising the temperature of ADP to $\sim 110 \mathrm{C}$ (the approximate maximum temperature for this crystal) raises the $90^{\circ}$ phase matching wavelength to $544.5 \mathrm{~nm}$. Conversely, cooling the crystal to its curie point $(v-120 \mathrm{c})$ will allow phase matching of wavelengths as short as $\sim 500 \mathrm{~nm}$. 


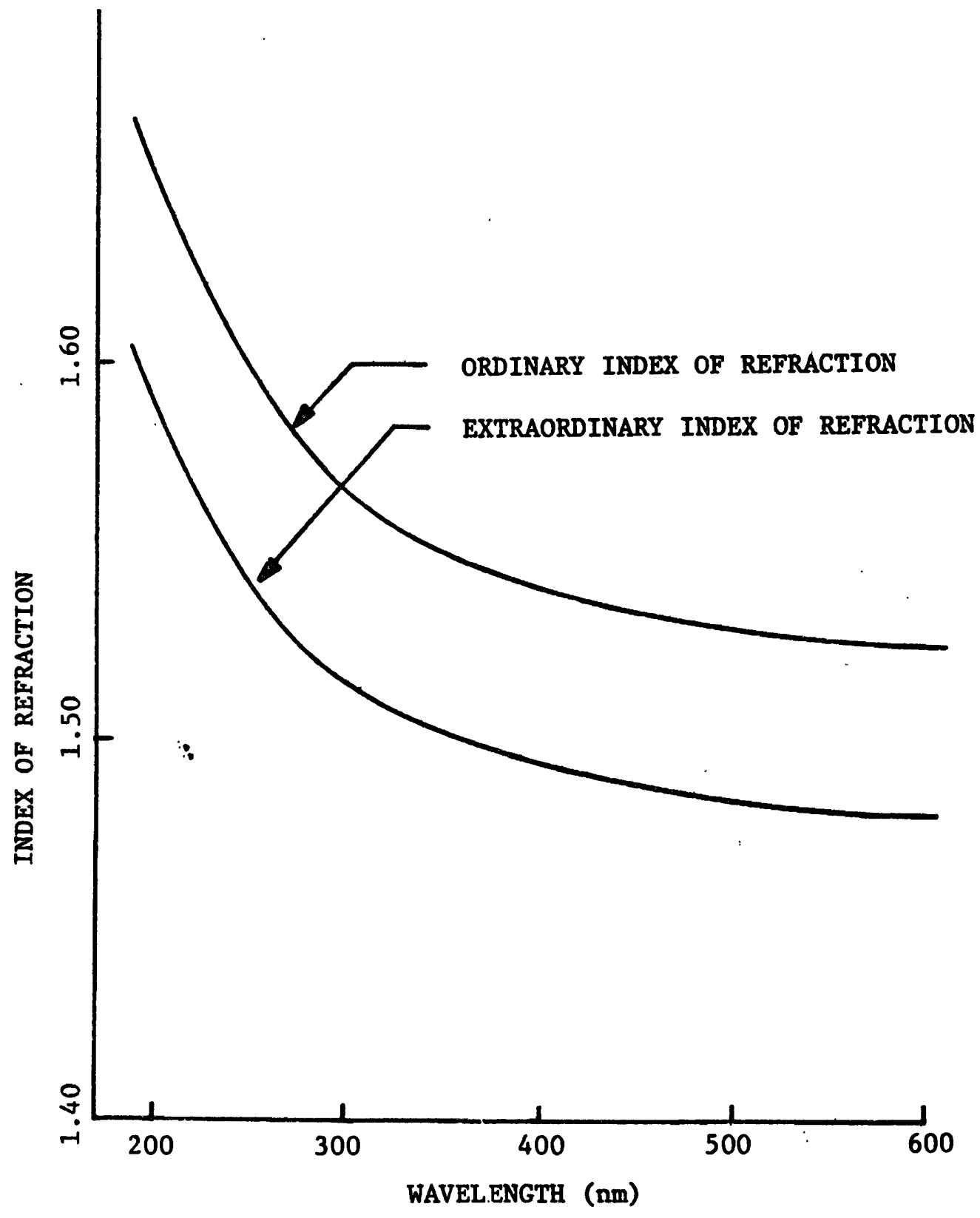

Fig. 35. Index of refraction as a function of wavelength for ADP. (Ref. 98.) 
In many cases it is desirable to phase match fundamental wavelengths which are longer than the long wavelength cutoff dictated by the crystal's decomposition temperature. Since the effect of increasing the temperature of a crystal such as ADP is to shift the extraordinary index curve closer to the ordinary index curve, it seems reasonable that if some other mechanism can be found which will also shift the extraordinary index, then that too might be a phase matching mechanism and longer wavelengths might be phase matched. A second method of phase matching can be found by changing the angle of propagation through the crystal. As was stated earlier, a light beam polarized parallel to the $x-y$ plane will see the crystal's ordinary index, whereas a beam polarized perpendicular to the $x-y$ plane will see the crystal's extraordinary index. A beam which is propagated between the $x-y$ plane and the $z$ axis (e.g. $E_{3}$, Fig. 34), sees an : index intermediate between the crystal's ordinary and extraordinary index. If one plots the index of refraction as a function of angle from the $z$ axis, the figure plotted will be an ellipse with a major axis radius $n_{0}$, and a minor axis radius $n_{e}$. Using the equation for an ellipse, the index of refraction as a function of angle is found to be given by: ${ }^{59}$

$$
\frac{1}{n(\theta)^{2}}=\frac{\cos ^{2}(\theta)}{n_{0}^{2}}+\frac{\sin ^{2}(\theta)}{n_{e}^{2}} \text {. }
$$

Equation (7.2) can be rewritten as:

$$
n(\theta)=\frac{n_{0} n_{e}}{\left(n_{e}{ }^{2} \cos ^{2}(\theta)+n_{0}{ }^{2} \sin ^{2}(\theta)\right)^{1 / 2}}
$$


Solving for $\sin ^{2}(\theta)$ as a function of $n(\theta), n_{0}$, and $n_{e}$, and recalling the phase matching condition, $n_{1}=n_{2}$, one can determine the phase matching angle for a given crystal and wavelength to be:

$$
\theta_{m}=\sin ^{-1}\left[\frac{\frac{1}{n_{01}^{2}}-\frac{1}{n_{02}^{2}}}{\frac{1}{n_{e 2}{ }^{2}}-\frac{1}{n_{02}^{2}}}\right]^{1 / 2}
$$

The phase matching angle versus wavelength for the crystal ADP is shown in Fig. 36. Changing the angle of propagation through a crystal permits phase matching to be achieved only for fundamental wavelengths which: are longer than the $90^{\circ}$ phase matchable wavelength at the same temperature.

\section{Choice of Crystal}

For any particular fundamental frequency there is generally more than one crystal which may be used to generate the second harmonic. For example, it is possible to frequency double, the light from a Rhodamine $6 G$ dye laser in temperature-tuned ammonium dihydrogen arsenate (ADA) and in angle-tuned ammonium dihydrogen phosphate (ADP) and potassium dihydrogen phosphate (KDP). ${ }^{101}$ A number of tradeoffs exist in determining which crystal should be used. Since it is difficult to rapidly change the temperature of a crystal having a volume of a few cubic centimeters, a temperature-tuned crystal must be ruled out, since a multiple beam measurement will necessitate being able to rapidly tune to a number of different wavelengths. Of the 


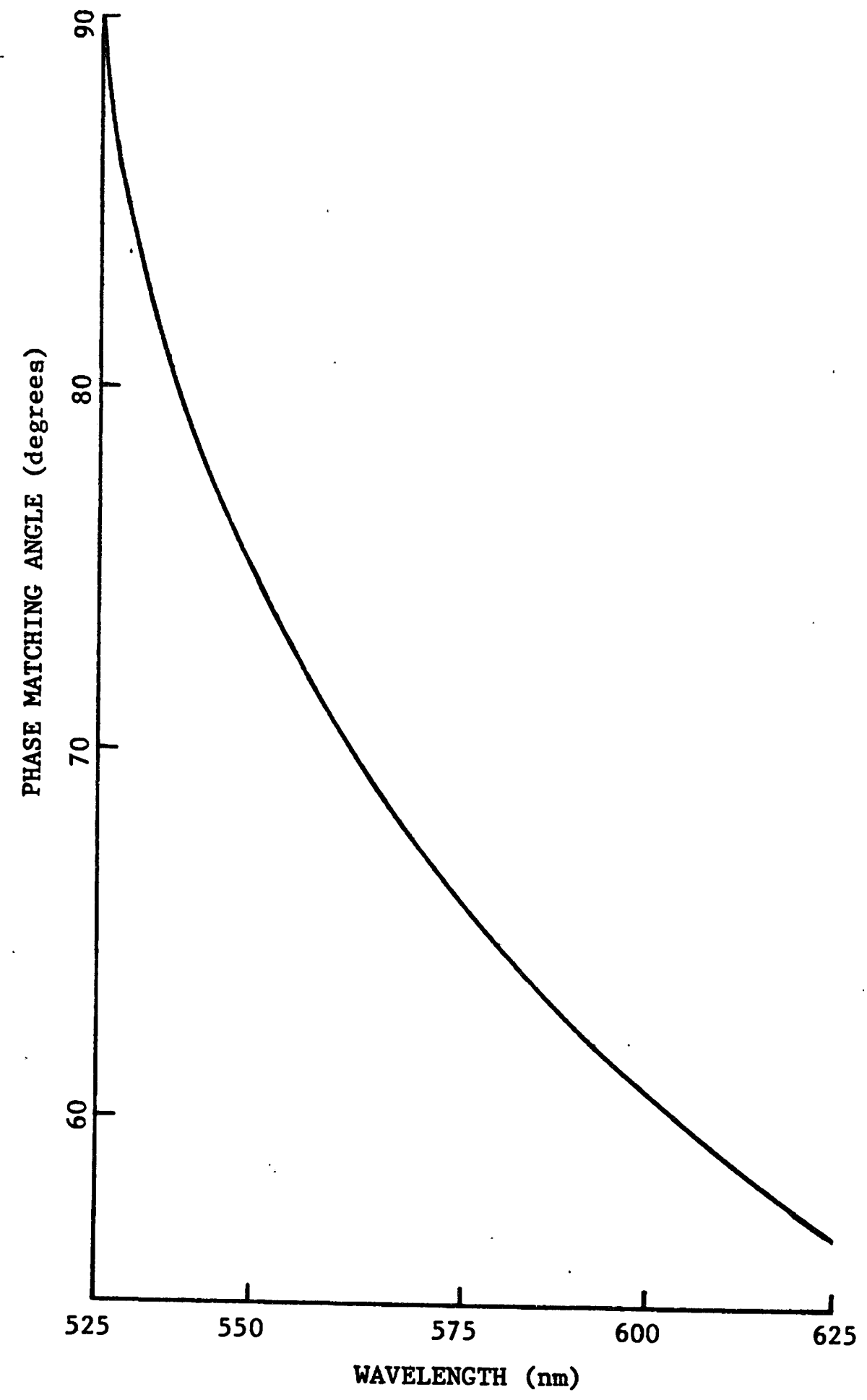
Fig. 36. Phase matching angle as a function of wavelength for
the crystal ADP. 
two angle tuned crystals, KDP is the best choice, since unlike ADP

its indices are insensitive to changes in ambient temperature. 100 $\mathrm{ADP}$ was chosen for the initial experiments, however, for the sole reason that at the beginning of the study it was available in large pieces, a few inches on a side, at low cost ( $\$ 40)$; whereas, a KDP frequency doubling crystal would have cost approximately $\$ 200$. Temperature considerations placed a small factor in the decision since the first instrument was to be used in the laboratory where the temperature is relatively constant.

\section{Frequency Doubling Considerations}

In this section two limitations upon the frequency doubling process which are peculiar to the angle-tuned method of achieving phase matching will be considered. The first question to be addressed is that of the angular tolerance upon the phase matching process. The second problem is termed walkoff; it will place a limit upon how tightly the input beam can be focussed.

Angle Tolerance on Phase Matching. Since phase matching was to be accomplished by changing the angle of the doubling crystal, it is wise to determine the sensitivity of the phase matching process to small angular errors in the adjustment of the frequency doubler. Returning once again to the phase matching condition for second harmonic generation, equation ( 7.1$)$ :

$$
\Delta \mathrm{k}=2 \pi\left(\frac{2 \mathrm{n}_{1}}{\lambda_{1}}-\frac{\mathrm{n}_{2}}{\lambda_{2}}\right)
$$

and taking a small variation about $\theta_{m}$, one gets: 


$$
\Delta \mathrm{k}\left(\theta_{\mathrm{m}}+\Delta \theta\right)=2 \pi\left[\frac{2\left(\mathrm{n}_{1}+\Delta \mathrm{n}_{1}\right)}{\lambda_{1}+\Delta \lambda_{1}}-\frac{\mathrm{n}_{2}+\Delta \mathrm{n}_{2}}{\lambda_{2}+\Delta \lambda_{2}}\right]
$$

But $\lambda_{2}=\frac{\lambda_{1}}{2}, \Delta \lambda_{2}=\frac{\lambda_{1}}{2}, \Delta \mathrm{n}_{1}=0$, which plus the fact that $k\left(\theta_{\mathrm{m}}\right)=0$ when substituted into $(7.8)$ gives:

$$
\begin{aligned}
\Delta \mathrm{k}(\Delta \theta) & =\frac{-4 \pi \Delta \mathrm{n}_{2}}{\lambda_{1}+\Delta \lambda_{1}} \\
& \simeq \frac{-4 \pi \Delta \mathrm{n}_{2}}{\lambda_{1}}
\end{aligned}
$$

But :

$$
\Delta \mathrm{n}_{2}=\frac{\mathrm{dn}_{2}(\theta)}{\mathrm{d} \theta} \Delta \theta
$$

where $\mathrm{n}_{2}$ is given by:

$$
\mathrm{n}_{2}(\theta)=\frac{\mathrm{n}_{\mathrm{o} 2} \mathrm{n}_{\mathrm{e} 2}}{\left[\mathrm{n}_{\mathrm{e}}{ }^{2} \cos ^{2}(\theta)+\mathrm{no}_{\mathrm{o}}{ }^{2} \sin ^{2}(\theta)\right]^{1 / 2}}
$$

Taking the required derivative, one gets:

$$
\Delta \mathrm{n}_{2}(\theta)=\frac{\mathrm{n}_{\mathrm{e} 2} \mathrm{n}_{\mathrm{O} 2}\left(\mathrm{n}_{\mathrm{e} 2}{ }^{2}-\mathrm{n}_{\mathrm{o} 2}{ }^{2}\right) \sin (2 \theta)}{2\left[\mathrm{n}_{\mathrm{o} 2}{ }^{2}+\left(\mathrm{n}_{\mathrm{e} 2}{ }^{2}-\mathrm{n}_{\mathrm{O} 2}{ }^{2}\right) \cos ^{2}(\theta)\right]} \Delta \theta
$$

The value of the quantity

$$
\frac{\sin ^{2}\left(\frac{\Delta k \ell}{2}\right)}{\left(\frac{\Delta k \ell}{2}\right)^{2}}
$$

in (7.1) equals $1 / 2$ when $\frac{\Delta k l}{2}= \pm 1.392$. This corresponds to the half width at half power points for the sinc function. The full width at 
half power is then given by:

$$
\frac{\Delta k \ell}{2}=2.784
$$

or

$$
\Delta k=\frac{5.568}{\ell}
$$

Equating (7.12) to (7.10) yields an expression for the full angular tolerance for phase matching of the doubling crystal. That relation is:

$$
\Delta \theta \ell=\frac{.886 \lambda_{1}\left[n_{02}{ }^{2}+\left(n_{e 2}{ }^{2}-n_{02}{ }^{2}\right) \cos ^{2}(\theta)\right]^{3 / 2}}{n_{e 2} n_{02} \sin (2 \theta)} .
$$

For example, in the spectral region of interest around $600 \mathrm{~nm}$, and for the crystal ADP, the following constants are applicable: ${ }^{98} \mathrm{n}_{{ }_{1}}=1.52402$, $\mathrm{n}_{\mathrm{o} 2}=1.56395, \mathrm{n}_{\mathrm{e} 2}=1.51277$, and $\theta=60.5^{\circ}$; and using these values in ( 7.13 ) one finds an angular tolerance of 0.005 milliradian-cm. This angular resolution can be provided by a rotary mechanism such as was described in Chapter $\mathrm{V}$ if driven by a stepping motor which turns in $0.18^{\circ}$ steps $^{102}$ a standard 40 TPI micrometer which in turn pushes against a 4 inch lever arm. The angular resolution of such a rotary device is 0.003 milliradians, which is sufficiently fine to allow one to automatically tune the frequency-doubler.

Walkoff. One of the problems which is encountered in angle tuned frequency doublers is that the fundamental beam and the second harmonic beam do not propagate in precisely the same direction within the crystal (see Fig. 37). The angle $\rho$ formed between the fundamental and second harmonic beams is given by: ${ }^{59}$ 


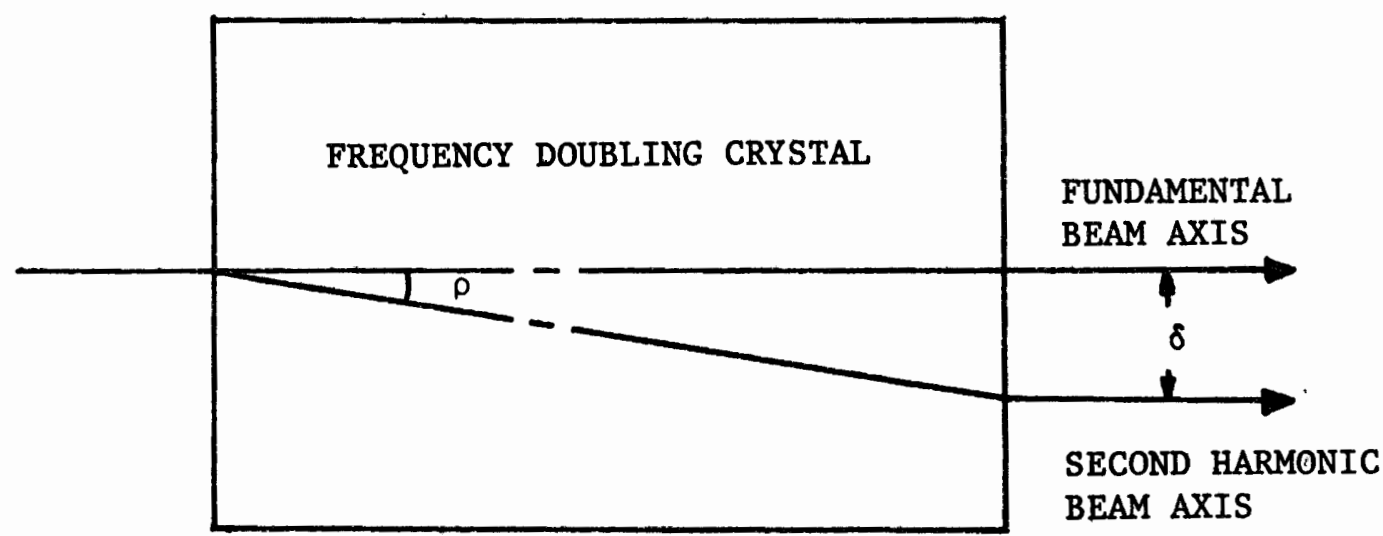

Fig. 37. Frequency doubling in an angle matched crystal illustrating walkoff between the fundamental and second harmonic beams. 


$$
\rho=\tan ^{-1}\left[\left(\frac{n_{o 2}}{n_{e 2}}\right)^{2} \tan \left(\theta_{m}\right)\right]-\theta_{m}
$$

If the fundamental beam is focussed to a small spot to increase the power density and thus increase the conversion efficiency, it is possible that the fundamental and generated second harmonic beams will walk off each other by the time they reach the end of the doubling crystal. Efficient frequency doubling, however, requires that the two beams remain overlapped over the length of the crystal, and a significant amount of walkoff can reduce the overall efficiency of the frequency conversion process. Thus walkoff and the necessity of . maintaining overlap between the two harmonic beams places a restriction on how tightly one can focus.

Boyd and Kleinman ${ }^{103}$ analyzed the problem of second harmonic generation in crystals with walkoff and derived the following expression for the second harmonic power in the presence of walkoff:

$$
P_{2}=\left(128 \pi^{2} \omega^{2} d^{2} P_{1}^{2} \ell\right) \cdot h / c^{4} n^{2}
$$

Equation (7.15) differs from (7.1) in that it is linear in crystal length, and the beam area $A$ is missing from the denominator. The missing length and area terms are concealed in a new, optimizable parameter $h$. While Boyd and Kleinman have determined exact values of $h$ for a variety of crystal lengths and walkoff angles, it is more important to note that in the region of parameters encountered when using angle-tuned crystals of the ADP family, h takes on a $1 / \sqrt{\ell}$. dependence. The net effect is a conversion efficiency expression which scales with the square root of the crystal length. Thus for maximum SHG conversion efficiency, it is still best to use as long a 
crystal as is practical, but the dependence of conversion efficiency on crystal length is not as great as is indicated by equation ( 7.1 ) which is for $90^{\circ}$ phase matching.

The Boyd and Kleinman analysis also indicates the amount of focussing of the pump beam into the crystal necessary to produce the maximum conversion of first harmonic to second harmonic. This occurs when the confocal distance $b$ of the focussed beam is approximately equal to the crystal length. The confocal distance is given by: 27

$$
b=\frac{2 \pi w_{0}^{2}}{\lambda}=l
$$

Under conditions of weak focussing, $(2 w / f l)>>1$, the focal length of the lens necessary to focus a beam of initial size w to a focussed size $w_{0}$, is given by: 24

$$
f \ell=\frac{\pi w_{0}}{\lambda}
$$

Solving (7.16) for $w_{0}$ in terms of crystal length and substituting into (7.17), the following expression for the optimum focal length lens is attained:

$$
f \ell=w\left[\frac{\pi l}{2 \lambda}\right]^{1 / 2}
$$

This is the focal length of a lens which will produce the maximum SHG efficiency in a crystal having walkoff.

As an example of the focal length which this expression predicts, consider a beam such as might be emitted by a rhodamine 6G dye laser: The wavelength will be taken to be $600 \mathrm{~nm}$, and from (6.21) the output beam diameter $2 \mathrm{w}$ is found to be $1.1 \mathrm{~mm}$. The optimum focal length lens for a $3.2 \mathrm{~cm}$ long crystal such as was actually used would be $160 \mathrm{~mm}$. 


\section{Spectral Acceptance of an Angle Matched Frequency Doubler}

For any single crystal temperature and orientation, only a

small range of input frequencies can be efficiently frequency doubled. The spectral acceptance of any particular crystal is determined by the amount of phase mismatch since efficient harmonic generation can take place only when $-\pi<\frac{\Delta k \ell}{2}<\pi$. As before

$$
\Delta k=2 \pi\left(\frac{2 n_{1}}{\lambda_{1}}-\frac{n_{2}}{\lambda_{2}}\right)^{2}
$$

where the subscripts 1 and 2 refer to the first and second harmonic quantities, respectively. Now assume a small perturbation $\Delta \lambda i_{1}$; then

$$
\Delta \mathrm{k}\left(\Delta \lambda_{1}\right)=\frac{4 \pi\left(\mathrm{n}_{1}+\Delta \mathrm{n}_{1}\right)}{\left(\lambda_{1}+\Delta \lambda_{1}\right)}-\frac{2 \pi\left(\mathrm{n}_{2}+\Delta \mathrm{n}_{2}\right)}{\left(\lambda_{2}+\Delta \lambda_{2}\right)}
$$

but $\lambda_{2}=\lambda_{1} / 2$, and $\Delta \lambda_{2}=\Delta \lambda_{1} / 2 ;$ therefore

$$
\begin{aligned}
\Delta k\left(\Delta \lambda_{1}\right) & =\frac{4 \pi\left(\mathrm{n}_{1}+\Delta \mathrm{n}_{1}\right)}{\left(\lambda_{1}+\Delta \lambda_{1}\right)}-\frac{2 \pi\left(\mathrm{n}_{2}+\Delta \mathrm{n}_{2}\right)}{\left(\frac{\lambda_{1}}{2}+\frac{\Delta \lambda_{1}}{2}\right)} \\
& \simeq 4 \pi\left[\frac{\Delta \mathrm{n}_{1}-\Delta \mathrm{n}_{2}}{\lambda_{1}}\right]
\end{aligned}
$$

The changes in index of refraction are given by $\Delta \mathrm{n}_{1}=\frac{d \mathrm{n}_{\mathrm{O}}}{\mathrm{d} \lambda} \Delta \lambda_{1}$, and $\Delta \mathrm{n}_{2}=\frac{\mathrm{dn} n_{2}}{\mathrm{~d} \lambda} \Delta \lambda_{2} . \quad \mathrm{n}_{2}$ is given by equation $(7.3)$.

$$
\begin{aligned}
\Delta \mathrm{k}=\frac{4 \pi}{\lambda_{1}}\left\{\frac{\mathrm{dn}_{\mathrm{o} 1}}{\mathrm{~d} \lambda}-\frac{1}{2}\left[\frac { \mathrm { dn } _ { \mathrm { o } 2 } } { \mathrm { d } \lambda } \left\{\frac{\mathrm{n}_{\mathrm{e} 2}}{\mathrm{~A}}-\frac{\mathrm{n}_{\mathrm{o} 2}{ }^{2} \mathrm{n}_{\mathrm{e} 2}}{\mathrm{~A}^{3}}(1-\cos (\theta)\}+\right.\right.\right. \\
\left.\frac{\mathrm{dn} \mathrm{e}_{2}}{\mathrm{~d} \lambda}\left\{\frac{\mathrm{n}_{\mathrm{o} 2}}{\mathrm{~A}}-\frac{\mathrm{n}_{\mathrm{o} 2} \mathrm{n}_{\mathrm{e} 2} \cos ^{2}(\theta)}{\mathrm{A}^{3}}\right\}\right] \Delta_{1}
\end{aligned}
$$


A is defined to be the quantity

$$
A \equiv\left[\mathrm{n}_{\mathrm{o} 2}{ }^{2}+\left(\mathrm{n}_{\mathrm{e}_{2}}{ }^{2}-\mathrm{n}_{\mathrm{o} 2}{ }^{2}\right) \cos ^{2}(\theta)\right]^{1 / 2}
$$

As before the value of the sinc function $\sin ^{2}\left(\frac{\Delta \mathrm{k} l}{2}\right) /\left(\frac{\Delta \mathrm{k} l}{2}\right)^{2}$ equals $1 / 2$ when $\frac{\Delta k \ell}{2}= \pm 1.392$, and the full width at half power is given by $\Delta k=5.568 / l$. Equating this expression for $\Delta \mathrm{k}$ to equation $(7.21)$, and using the parameter values for ADP of: $\theta=60.5^{\circ}, \lambda_{1}=600 \mathrm{~nm}, \mathfrak{n}_{01}=$ $1.52402, \mathrm{n}_{\mathrm{o} 2}=1.56395, \mathrm{n}_{\mathrm{e} 2}=1.51277, \frac{\mathrm{d} \mathrm{n}_{\mathrm{O} 1}}{\mathrm{~d} \lambda_{1}}=-.513899 \times 10^{-5}$, $\frac{\mathrm{dno2}}{\mathrm{d} \lambda 2}=-3.70341 \times 10^{-4}$, and $\frac{\mathrm{d} \mathrm{e}_{\mathrm{e}}}{\mathrm{d} \lambda_{2}}=-3.25168 \times 10^{-4}$, one finds a half power spectral tolerance per $\mathrm{cm}$ of crystal length of $0.16 \mathrm{~nm}-\mathrm{cm}$.

\section{Frequency Doubler Fabrication}

A crystal of ADP measuring $\sim 2 \mathrm{~cm} \times 1 \mathrm{~cm} \times 3.2 \mathrm{~cm}$ was cut from a large crystal of ADP supplied by EDO Western of Salt Lake City, Utah using a wire saw. The long direction of the crystal was oriented $\sim 60$ degrees from the optic axis in accordance with phase matching conditions. The direction of the optic axis was determined by observing the Maltese cross pattern through a pair of crossed polarizers. The rough cut crystal faces were polished first using a slurry of number 600 silicon carbide and ethylene glycol on glass followed by a final polishing using a slurry of cerium oxide powder and ethylene glycol on a lap of black sealing wax sold by Van Waters and Rogers. The slurry was removed from the polished surface of the crystal by carefully wiping with facial tissue. This final stage of polishing was accomplished using cerium oxide on a lap surface which was virtually dry to avoid fogging of the crystal by any water which might have been present in the glycol. 
The polished crystal was then attached to a rotary table whose motian could be controlled by the rotation of a micrometer screw. In this way the crystal could be rotated in a plane formed by the $z$ axis of the crystal and a $45^{\circ}$ angle between the $x$ and $y$ crystallographic axis, thus tuning the frequency doubler. 
CHAPTER VIII

PERFORMANCE OF THE FLASHLAMP-PUMPED FREQUENCY-DOUBLED DYE LASER

In this chapter the performance of the frequency-doubled flashlamp-pumped dye laser described in the preceding chapters will be summarized, and the actual operational performance compared with what was expected from theoretical consideration.

\section{Dye Laser Operating Characteristics}

The dye laser was finally operated in the configuration shown in Fig. 38. Using the one atmosphere argon-filled flashlamp, the laser could be operated at an input energy of $25 \mathrm{~J}$ and a PRF of 1 Hz for a period of about 3 hours before the flashlamp would fail explosively, terminating laser operation. Flashlamp failure was coincident with the buildup of silicon deposits around the electrodes and the resultant stoppage of the flow of fresh argon. The $25 \mathrm{~J}$ and $1 \mathrm{~Hz}$ combination of flashlamp input energy and pulse rate was used for most subsequent measurements since 3 hours was rough1y the amount of time necessary to complete all of the measurements on the dye laser and frequency doubler, and increasing either of the afore-mentioned quantities reduced the total operating time of the laser before flashlamp failure. The flashlamp light pulse was longer than calculated or desired but was short enough to allow broadband lasing to occur with a slope efficiency of 0.2 percent, and an overall energy efficiency of 0.1 percent at $40 \mathrm{~J}$ of input energy as can be deduced from $\mathrm{Fig} .27$. 


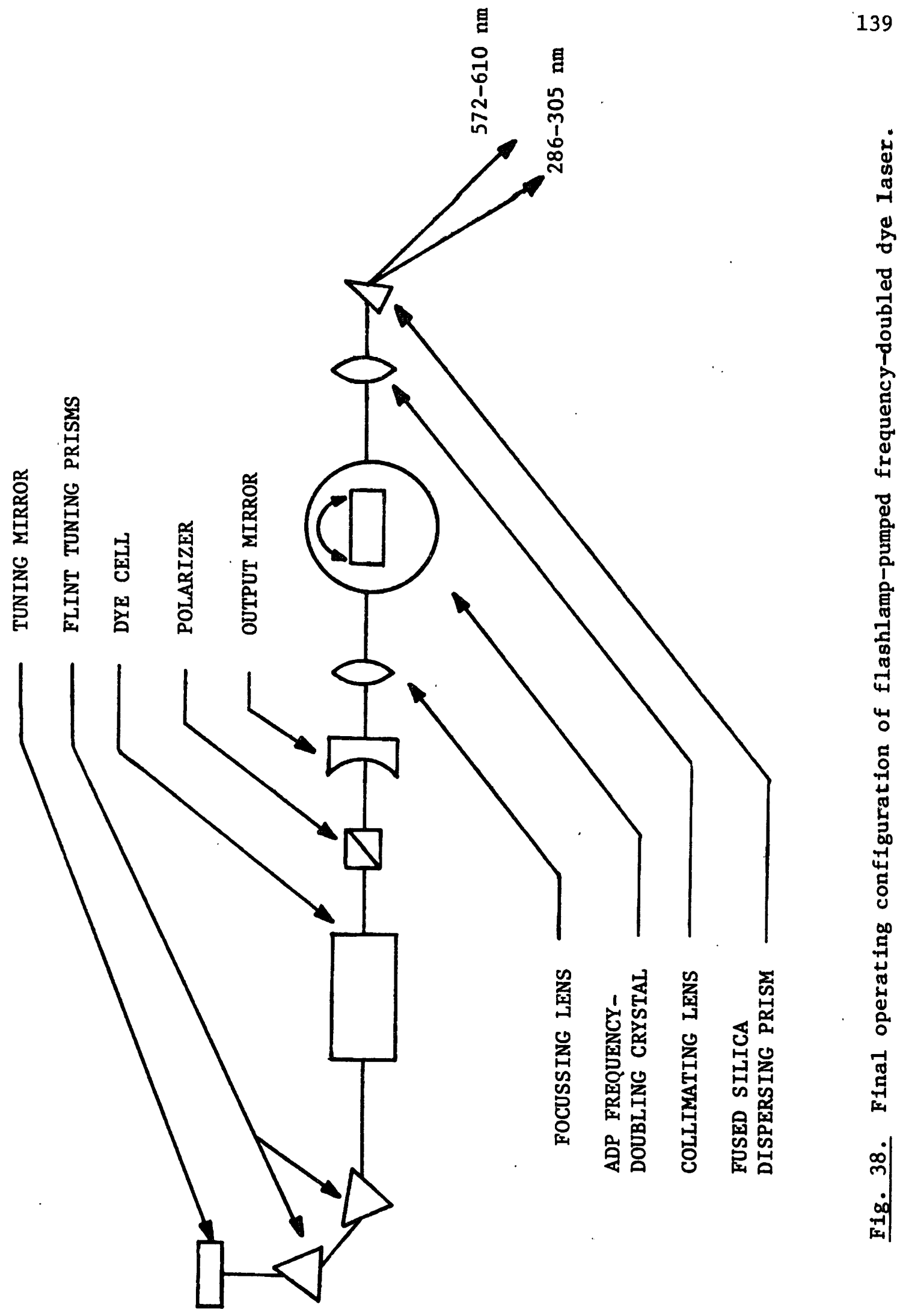


When two flint glass tuning prisms were inserted inside the cavity, the laser efficiency dropped to 0.003 percent, a factor of 20 down from the untuned configuration at the same energy input level of $25 \mathrm{~J}$. The reason for this drastic reduction in efficiency is not clear. Nevertheless, with an input of $25 \mathrm{~J}$ the prism-tuned dye laser did put out nearly a millijoule of energy at $585 \mathrm{~nm}$, the wavelength of maximum output for rhodamine $6 \mathrm{G}$ when flashlamp pumped. A tuning curve showing the dye 1aser's output energy as a function of output wavelength is shown in Fig. 39, for a constant input energy of $25 \mathrm{~J}$ and a PRF of $1 \mathrm{~Hz}$. The dye laser output wavelength was measured using a Jarrell Ash $1 / 4 \mathrm{~m}$ monochrometer which was accurate to $\pm 0.5 \mathrm{~nm}$. The dye laser energy output was measured using a calibrated Hewlett Packard HP4203. PIN photodiode placed approximately 2 inches behind a ground fused silica diffuser. The diffuser reduced the power incident upon the detector and also made the detector less sensitive to the incident beam's direction and position. The detector was used in the circuit shown in Fig. 40. The photodiode had been calibrated at the Oregon Graduate Center using a chopped argon ion laser beam whose average output power was measured using a Coherent Radiation Model 201 optical power meter. The length of the dye laser output pulse was $2 \mu \mathrm{S}$ full width at half maximum as measured using a United Detector PIN $10 \mathrm{D}$ silicon photodiode: the actual pulse shape is shown in Fig. 41. The dye laser linewidth was measured to be $0.5 \pm 0.1 \mathrm{~nm}$ using a Fabry Perot etalon with a free spectral range of $1 \mathrm{~nm}$ and a finesse of 10. A beam expanding tele- 


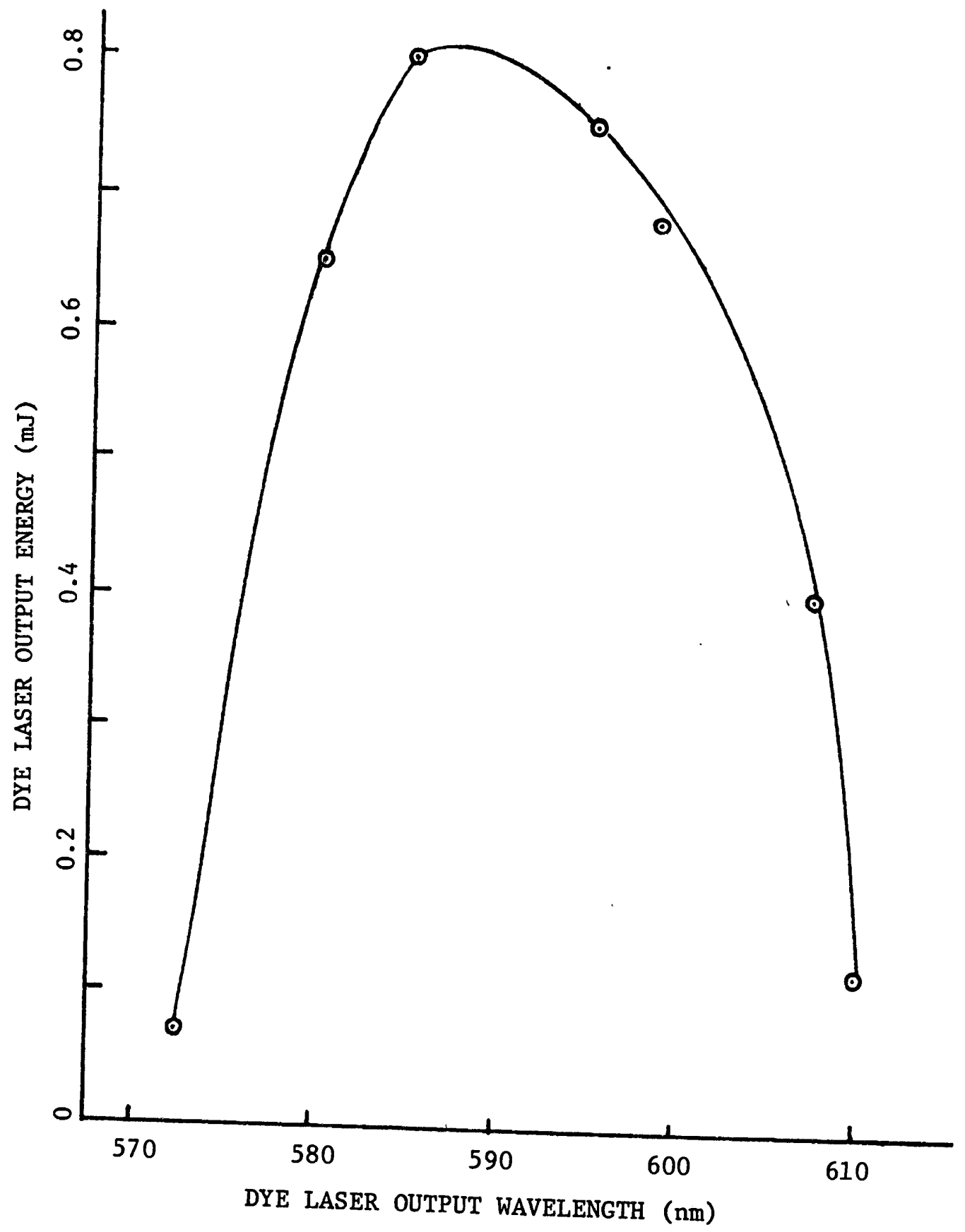

Fig. 39. Rhodamine 6 G prism-tuned dye laser output as a function of wavelength with a constant electrical input energy of $25 \mathrm{~J}$. 


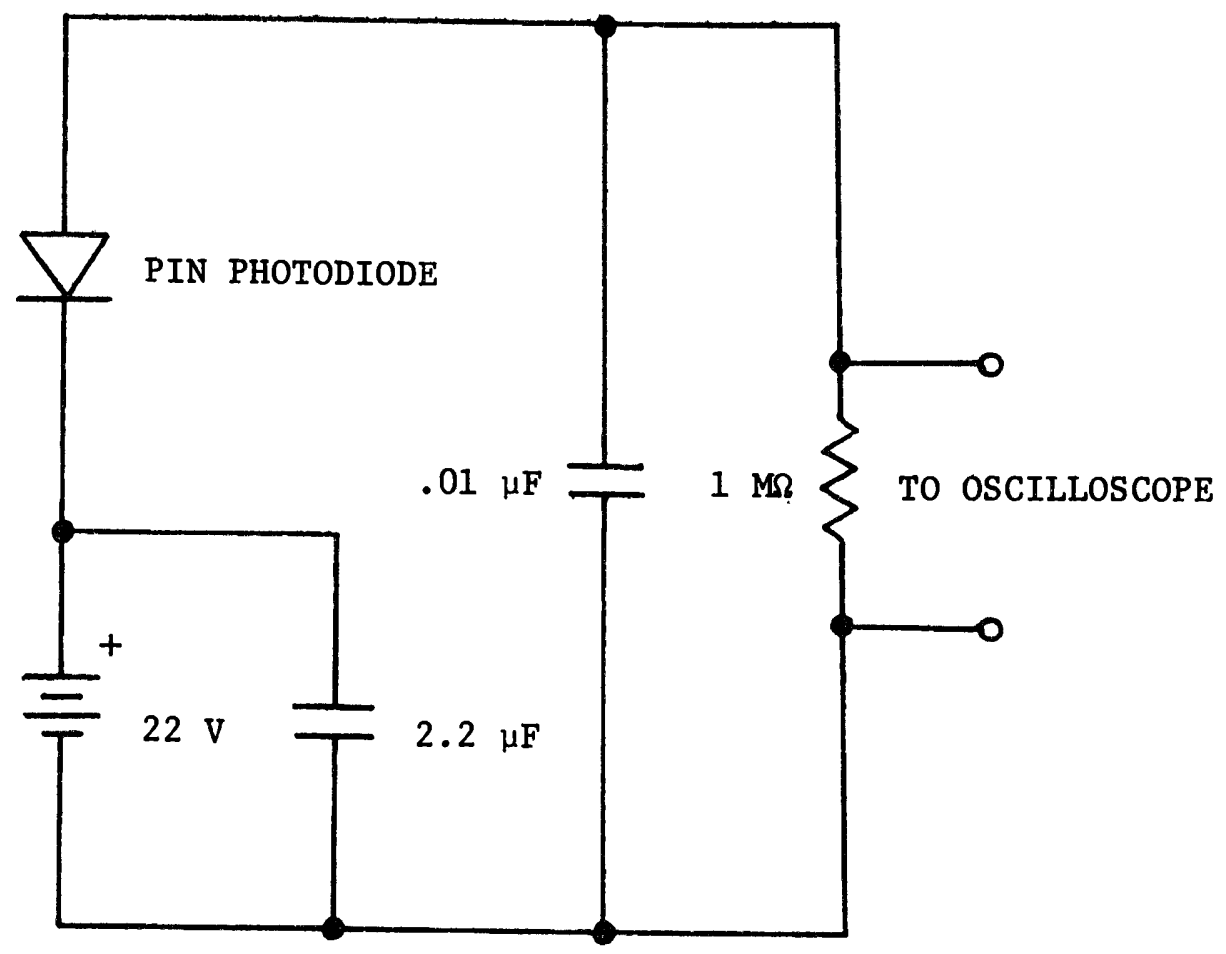

Fig. 40. Integrating photodiode circuit used to measure dye laser output energy. 


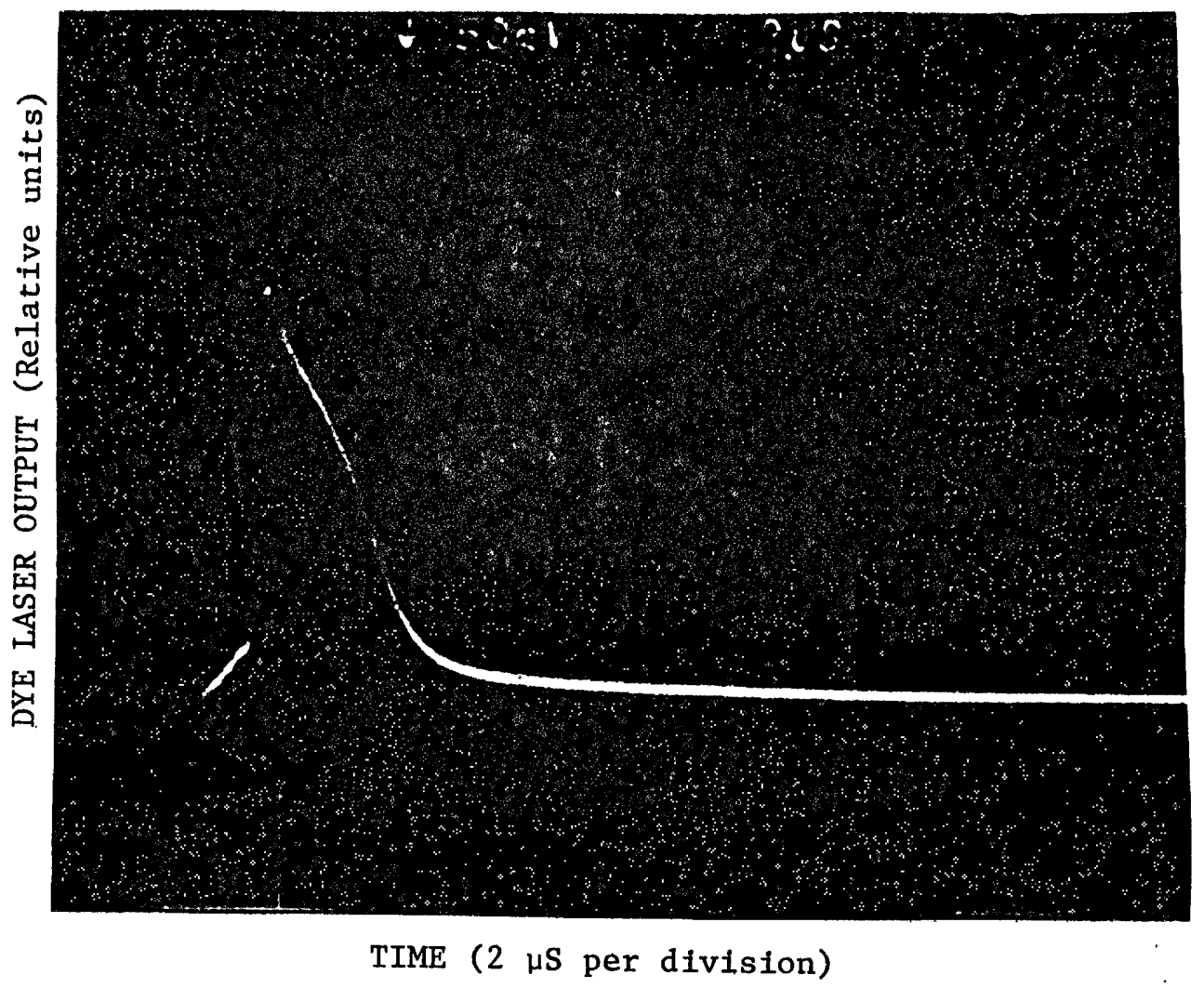

Fig. 41. Output pulse from flashlamp pumped dye laser. 
scope would have reduced the linewidth by 10 had it been available.

\section{Frequency Doubler Performance}

The light from the dye laser was focussed into the angle tuned ADP frequency doubler using a $185 \mathrm{~mm}$ focal length lens. This lens has a longer focal length than the optimum of $160 \mathrm{~mm}$ which was calculated using ( 7.18$)$; however, $185 \mathrm{~mm}$ represented the closest focal length lens available at the time of the experiment. The output beams from the crystal were recollimated using a $125 \mathrm{~mm}$ focal length fused silica lens before being separated by a fused silica dispersing prism. The second harmonic energy was measured using a Quantrad 100 UV-PV-RM PIN photodiode which had been calibrated at $266 \mathrm{~nm}$ and $355 \mathrm{~nm}$ using the third and fourth harmonics of a Nd:YAG laser. The photodiode circuit was the same as was used to measure the dye laser visible energy (see Fig. 40). The second harmonic energy as a function of wavelength is shown in Fig. 42. As can be seen, the efficiency of the second harmonic generating process is on the order of 0.1 percent. The reason for the low efficiency is probably due to the large linewidth of the dye laser $(\sim 0.5 \mathrm{~nm})$ in comparison the rather narrow spectral acceptance of the ADP crystal. ( $0.04 \mathrm{~nm})$.

To determine how well the experimental second harmonic results agree with theory, let us return to (7.1) and assuming perfect phase matching, then:

$$
P_{2}=\frac{52.2 d^{2} \ell^{2} P_{1}^{2}}{n^{3} \lambda^{2} A}
$$


145

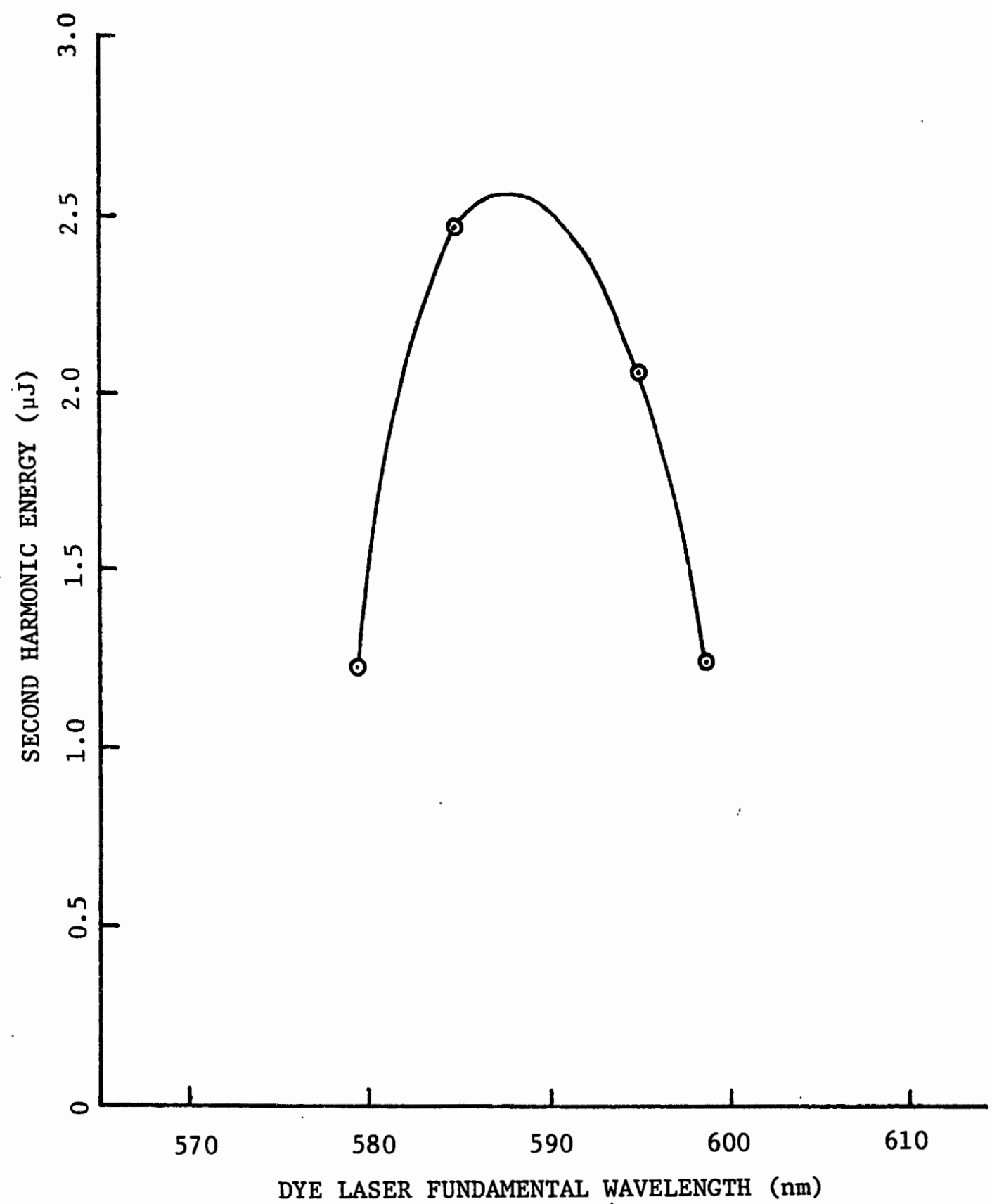

Fig. 42. Second harmonic energy as a function of wavelength for a constant input electrical energy of $25 \mathrm{~J}$. 
or for efficiency:

$$
n=\frac{52.2 \mathrm{~d}^{2} \ell^{2} \mathrm{P}_{1}}{\mathrm{n}^{3} \lambda^{2} \mathrm{~A}}
$$

The peak fundamental power is given by:

$$
P=\frac{\text { dye laser energy }}{\text { laser pulse duration }}
$$

At $590 \mathrm{~nm}$, the dye laser put aut $\sim 0.8$ millijoules in a pulse which was roughly $2 \mu \mathrm{S}$ long, thus yielding a peak power of $P_{1} \sim 800 \mathrm{~W}$. The beam area can be calculated using the value of $w_{0}$ found in (7.17), and is given by:

$$
\begin{aligned}
A & =\pi w_{0}{ }^{2} \\
& =\frac{\lambda^{2}(f l)^{2}}{\pi w_{0}{ }^{2}}
\end{aligned}
$$

Using as values for the parameters: $\lambda=.59 \times 10^{-4} \mathrm{~cm}, f \ell=18.5 \mathrm{~cm}$, $w=.055 \mathrm{~cm}$, we get a beam area of $A=1.3 \times 10^{-4} \mathrm{~cm}^{2}$. This is true only if the laser is operating in its lowest order transverse mode. This was not the case; in fact, it was capable of sustaining up to third order modes as determined by the size of the limiting aperature relative to the fundamental order beam size. Since the effect of increasing mode number is to increase the spot radius at the focus by the factor $\sqrt{2 M+1}$ where $M$ is the transverse mode order, the actual beam area will be larger by a factor of 7 or $\mathrm{A}_{\text {actual }}=$ $9.2 \times 10^{-4} \mathrm{~cm}^{2}$. Using this area in (8.1) together with $\mathrm{d}=1.36 \mathrm{x}$ $10^{-9}$ esu, $l=3.2 \mathrm{~cm}, \mathrm{P}_{1}=80 \mathrm{w}, \mathrm{n}=1.51, \lambda=5.9 \times 10^{-5} \mathrm{~cm}$, one gets an estimated efficiency of 2.8 percent.

The measured output of the dye laser was $0.8 \mathrm{~mJ}$ at $590 \mathrm{~nm}$, 
while the frequency doubled energy was $2.5 \mu \mathrm{J}$. If the assumption is again made that only ten percent of the dye laser's output energy lies within the spectral acceptance of the frequency doubling crystal. then the effective dye laser output energy is $80 \mu \mathrm{J}$. The effective frequency doubler conversion efficiency is 3.1 percent which is In good agreement with the calculated value of 2.8 percent. 


\section{CHAPTER IX}

\section{CONCLUSIONS AND RECOMMENDATIONS}

The purpose of this study was to determine the feasibility of developing a low-cost, reliable monitoring system to measure the average concentration of sulfur dioxide over a long path. The requirements of such an instrument have been analyzed, and a system has beeri designed based on the principle of absorption spectroscopy. Sufficient sensitivity and selectivity have been found to be achievable through the use of a tunable laser light source operating in the ultraviolet. Three phenomena that could strongly influence the operation of the system have been determined to be spectral interference, scattering, and turbulence. The influence of these effects has been analyzed in a preliminary fashion and found to be acceptable.

In order to determine the effectiveness of the selected approach, a frequency-doubled dye laser light source has been designed, constructed, and tested in the laboratory. The relatively high system cost coupled with short flashlamp lifetimes suggests that it would be most difficult to achleve the low cost and reliability necessary for such an instrument to be acceptable to a typical air pollution agency. Improving the reliability would likely further increase the cost.

It should be stressed that the system designed and analyzed appears quite feasible, but not within the given constraints. If higher cost is acceptable, then the following modifications to the dye laser are suggested as ways of improving its efficiency and therefore increasing 
the flashlamp lifetime:

1. Reduce the intracavity optical losses by antireflection coating the dye cell windows.

2. Decrease the diameter of the dye cell until it is more nearly the size of a fundamental mode laser beam. In this way less of the flashlamp light will be absorbed by dye outside of the lasing region.

3. Improve both the optical quality and reflectivity of the reflectors used to couple the flashlamp light into the dye cell.

A second portion of the dye laser which needs significant Improvement is that of spectral narrowing. Introduction of an etalon into the cavity is the surest way to achieve spectral narrowing; however, it pill be necessary to tune the etalon to each desired wavelength and this w111 probably necessitate the use of another stepping motor driven rotary mount. A simpler method of decreasing the laser linewidth is to introduce a beam expanding telescope between the dye cell and the tuning prisms. The cost of such a telescope will not be small since the optical elements should be of diffraction limited quality and antireflection coated.

A third change which will probably be necessary eventually is the abandoment of the triggered spark gap in favor of a thyratron. The amount of radio frequency interference (RFI) generated by the spark gap is sufficient to render most test equipment useless, and it can probbbly be expected to do the same thing to the pollutant detection electronics: The frequency doubler design is basically soind, but the following improvements are suggested: 
1. Replacing the $185 \mathrm{~mm}$ focal length lens with one of more optimum focal length.

2. Acquiring a KDP crystal to replace the present, temperature sensitive $\mathrm{ADP}$.

3. Use of a dichroic mirror to separate the fundamental and second harmonic wavelengths. With the present fused silica dispersing prism, the angle that the uv leaves the setup changes as one changes wavelength.

In addition to the necessary additional laser system development, the effects of turbulence on the system design requirements and performance should be examined In more detail. In fact, that would appear to be a major study in itself.

Finally, recent rapid advances in the state-of-the-art of microprocessors suggests that a new study of detection methods for reducing the effects of spectral interference be undertaken. It might well turn out that through advanced and now practical computational techniques one could deduce the pollutant concentration from data produced by a single multiline infrared gas laser. 
REFERENCES

1. Morgan, G.B., Ozolins, G., and E.C. Taber, "Air Pollution Surveillance Systems," Science 170, 289 (1970).

2. Pescar, R.E. and Hartman, C.H., "Automated Gas Chromatographic Analysis of Sulfer Pollutants" in Air Quality Instrumentation, Vol. 1, J.W. Scales, Ed. (Instrument Society of America, 1972), pp. 89-113.

3. Federal Register, November 25, 1971.

4. Stevens, R.K., O'Keeffe, A.E., and Ortman, G.C., "Current Trends in Continuous Air Pollution Monitoring Systems," in Air Quality Instrumentation, Vo1. 1, J.W. Scales, Ed. (Instrument Societyi of America, 1972), pp. 89-113.

5. Bauman, R.P., Absorption Spectroscopy (Wiley, 1962).

6. Thompson, R.T. Jr., Hoel1, J.M. Jr., and Wade, W.R., "Measurements of $\mathrm{SO}_{2}$ Absorption Coefficients Using a Tunable Dye Laser," J. of App. Phys. 46, p. 3040 (1975).

7. Halliday, D., and Resnick, R., Physics, (Wiley, 1962), p. 1061.

8. Liberman, I., "Incoherent Optical Sources," in Handbook of Lasers, R.J. Pressley, Ed., (Chemical Rubber Company, 1971), p. 14.

9. Klein, M.V., Optics, (Wiley, 1970), p. 129.

10. Photomultiplier Tubes, (RCA, 1971), p. 28.

11. Limperis, T., "Detectors," in Handbook of Military Infrared Technology, W.L. Wolfe, Ed., (Office of Naval Research, 1965), p. 477 .

12. Kildal, H., and Byers, R.L., "Comparison of Laser Methods for the Remote Detection of Atmospheric Pollutants," Proc. IEEE 59, 1644 (1971).

13. Menzies, R.T., George, N. and Bhaumic, M.L., "Spectral Coincidences Between Emission Lines of the CO Laser and Absorption Lines of Nitrogen Oxides," IEEE J. Quan. Elec. QE-6, 800 (1970).

14. Kreuzer, L.B., Kanyon, N.D., and Patel, C.K.N., "Air Pollution: Sensitive Detection of Ten Pollutant Gases by Carbon Monoxide and Carbon Dioxide Lasers," Science 177, 347 (1972).

15. Antcliffe, G.A. and Wrobel, J.S., "Detection of Gaseous Pollutant Sulfer Dioxide Using Current Tunable $\mathrm{Pb}_{1-\mathrm{x}} \mathrm{Sn}_{\mathrm{x}}$ Te Diode Lasers," App 1. Opt. 11, 1548 (1972). 
16. Hanst, P.L., "Spectroscopic Methods for Air Pollution Analysis," in Advances in Environmental Science and Technology, Vol. 2, J.N. Pitts and R.L. Metcalf, Eds. (Wiley-Interscience, 1971), p. 91 .

17. Plass, G.N. and Yates, H., "Atmospheric Phenomena, in Military Handbook of Infrared Technology, (Office of Naval Research, 1965), p. 189.

18. Snowman, L.R. and Gillmeister, R.J., "Infrared Laser System for Extended Area Monitoring of Air Pollution," presented at the Joint Conference on Sensing of Environmental Pollutants, Palo Alto, California, November 8-10, 1971.

19. Barringer, R., and B.C. Newberry, "Remote Sensing Correlation Spectrometry for Pollution Measurement," Ninth Conference on Methods in Air Pollution and Industrial Hygiene Studies, Pasadena, California, February, 1968.

20. Eppers, W., "Atmospheric Transmission," Handbook of Lasers With Selected Data on Optical Technology, R. Pressley, Ed., (Chemical Rubber Company, 1971), p. 41.

21. Kerr, J.R., Titterton, P.J., Kraemer, A.R., and Cooke, C.R.; "Atmospheric Optical Communications Systems," Proceedings of the IEEE 58, 1691 (1970).

22. Lawrence, R.S. and Strobehn, J.W., "A Survey of Clean Air Propagation Effects Relative to Optical Communications," Proceedings of the IEEE 58, 1523 (1970).

23. Davis, J.I., "Consideration of Atmospheric Turbulence in Laser Systems Design," Applied Optics $\underline{5}, 139$ (1966).

24. Kogelnik, H. and Li, T., "Laser Beams and Resonators," Applied Optics $\underline{5}, 1550$ (1966).

25. Siegman, A.E., An Introduction to Lasers and Masers, (McGraw-Hi11, 1971), p. 313 .

26. Tatarski, V.I., Wave Propagation in a Turbulent Medium, (McGrawHi11, 1961).

27. Lipson, C., and Sheth, N., Statistical Design and Analysis of Engineering Experiments, (McGraw-Hi11, 1973), p. 74.

28. Tucker, H.G., An Introduction to Probability and Mathematical Statistics, (Academic Press, 1962), p. 102.

29. Pincus, P., Oregon Graduate Center, Beaverton, Oregon, Private Communication. 
30. Tennekes, H. and Wyngaard, J.C., "The Intermittent Sma11Scale Structure of Turbulence: Data Processing Hazards," J. Fluid. Mech. 55, p. 93 (1972).

31. Jaff'e, H.H. and Orchin, M., Theory and Applications of Ultraviolet Spectroscopy, (Wiley, 1962), p. 3 .

32. Seinfeld, J.H., Air Pollution, (McGraw-Hill, 1975), p. 28.

33. Zaidel', A.N., and Shreider, E.Y., Vacuum Ultraviolet Spectroscopy, (Ann Arbor-Humphrey Science Publishers, 1970), p. 281 .

34. Tsongas, G.A., Portland State University, Portland, Oregon, Private Communication.

35. Melfi, S.H., "Remote Sensing for Air Quality Management," in Laser Monitoring of the Atmosphere, (S.pringer-Verlag, $1976)$, p. 15 .

36. Clements, J.H., "On the Absorption Spectrum of Sulpher Dioxide," Physical Review, 47, 22A (1935).

37. Hal1, T.C. and Blacet, F.E., "Separation of the Absorption Spectra of $\mathrm{NO}_{2}$ and $\mathrm{N}_{2} \mathrm{O}$ in the Range of 2400-5000 A," Journal of Chemical Physics, 20, 1745 (1952).

38. Griggs, M. "Absorption Coeffiecients of Ozone in the U1traviolet and Visible Regions," J. of Chem. Phys. 49, 857 (1968).

39. O'Shea, D.C. and Dodge, L.G., "NO, Concentration Measurements in an Urban Atmosphere Using Differential Absorption Techniques," App. Opt. 13, 1481 (1974).

40. Herzberg, G., Spectra of Diatomic Molecules, (Van Nostrand, 1950), p. 54 .

41. Wolfe, W.L., Handbook of Military Infrared Technology, (Office of' Naval Research, 1965), p. 228.

42. O'Neil1, F., and Whitney, W.T., "A High Power Tunable Laser for the 9-12.5 m Spectral Range," App. Phys. Lett. 31, 270 (1977).

43. Pollack, M.A., "Molecular Gas Lasers," Handbook of Lasers, (The Chemical Rubber Co., 1971), p. 329.

44. Gerry, E.I. and Leonard, D.A., "Measurement of $10.6 \mu \mathrm{CO}_{2}$ Laser Transition Probability and Optical Broadening Cross Sections," Applied Physics Letters 8, 227 (1966). 
45. Bridges, T.J., Burkhardt, E.G., and Smith, P.W., " $\mathrm{CO}_{2}$ Waveguide Lasers," Applied Physics Letters 26, 403 (1972).

46. Panish, M.B., "Heterostructure Injection Lasers," Proc. IEEE 64, 1512 (1976).

47. Pankoye, J.I., "Injection Lasers," Handbook of Lasers with Selected Data on Optical Technology, R. Pressley, Ed., (Chemica1 Rubber Co., 1971), p. 365.

48. Laser Analytics, Lexington, MA, 02173, Ibid.

49. Laser Focus Buyers Guide, (Advanced Technology Publications, Inc., 1977), p. 165.

50. Harris, S.E., "Tuneable Optical Parametric Oscillators," Proceedings of the IEEE 57, 2096 (1969).

51. "Laser Selection Guide," Electra-Optical Systems Design, Dec. 1973, p. 25.

52. "Sophisticated Sensor Targets Pollutants," Oregonian, April 23, 1978.

53. Patel, C.K.N. and Shaw, E.D., "Tunable Stimulated Raman Scattering From Mobile Carriers in Semiconductors," Phys. Rev. B. $\underline{3}, 1279$ (1971).

54. Hinkley, E.D., Nill, K.W., and Blum, F.A., "Tuneable Lasers in the Infrared," Laser Focus, April 1976, p. 49.

55. Snavely, B.B., "Dye Lasers," Journal of Society of Photo-Optical Instrumentation Engineers 8 , 119 (1970).

56. Furumoto, H.W. and Ceccon, H.L., "Ultraviolet Organic Liquid Lasers," IEEE Journal of Quantum Electronics QE-6, 262 (1970).

57. Searles, S.K. and Hart, G.A., "Stimulated Emission at $281.8 \mathrm{~nm}$ from XeBr," Appl. Phys. Lett. 27, 243 (1975).

58. Kudryavtsev, Y.A. and Kuz'mina, N.P., "Excimer Ultraviolet Gas-Discharge XeF, XeC1, and KrF Lasers," Sov. J. Quantum Elect. I, 131 (1977).

59. Zernike, F. and Midwinter, J.E., Applied Nonlinear Optics (John Wiley, 1973), p. 102.

60. Dunning, F.B., Tittel, F.K., and Stebbings, R.F., "The Generation of Tuneable Coherent Radiation in the Wavelength Range 2300-3000 A Using Lithium Formate Monohydride," Optics Communications I, 181 (1973). 
61. Dewey, C.F., Cook, W.R., Hodgson, R.T., and Wynne, J.J.,

"Frequency Doubling in $\mathrm{KB}_{5} \mathrm{O}_{8}-4 \mathrm{H}_{2} \mathrm{O}$ and $\mathrm{NH}_{4} \mathrm{~B}_{5} \mathrm{O}_{8}-4 \mathrm{H}_{2} \mathrm{O} \mathrm{ti}$

$217.3 \mathrm{~nm}, "$ Applied Physics Letters 26, $744^{5}$ ( 1975$\}$.

62. Eastman Kodak Company, Data Release No. JN-169.

63. Amman, E.O., Decker, C.D., and Falk, J., "High-Peak-Power $532 \mathrm{~nm}$ Pumped Dye Laser," IEEE Journal of Quantum Electronics. QE-10, P. 463 (1974).

64. Hansch, T.W., "Repetitively Pulsed Tuneable Dye Laser for High Resolution Spectroscopy," Applied Optics 11, 895 (1972).

65. Hansch, T.W., Schawlow, A.L. and Toschek, P., "Simple Dye Laser Repetitively Pumped by a Xenon Ion Laser," IEEE Journa1 of Quantum Electronics QE-9, 553 (1973).

66. Peterson, O.G., Tuccio, S.A., and Snavely, B.B., "CW Operation of an Organic Dye Solution Laser," Applied Physics Letters 17, 245 (1970).

67. Snavely, B.B., "Flashlamp Excited Organic Dye Lasers," Proceedings of the IEEE 57, 1374 (1969).

68. Molectron Dye List, August 1975.

69. Gilbert, R.W., "Coherent Anti-Stokes Raman Spectroscopy Using Tuneable Dye Lasers Pumped by an Ultraviolet Nitrogen Lser," Masters Thesis, Naval Postgraduate Schoo1, 1975.

70. Itzkan, I. and Cunningham, F.W., "Oscillator Amplifier Dye Laser System Using $N$ Laser Pumping," IEEE Journal Quantum Electronics 8 , 101 ( 1972$)$.

71. Sutton, D.G. and Cappelle, G.A., "KrF-Laser-Pumped Tunable Dye Laser in the U1traviolet," App. Phys. Lett. 29, p. 563 (1976).

72. Pappalardo, R., Samuelson, H., and Lempicki, A., "Calculated Efficiency of Dye Lasers As a Function of Pump Parameters and Triplet Lifetime," Journal of Applied Physics 43, 3776 (1972).

73. Mack, M.E., "Optical Design Problems in Dye Lasers," SPIE 69.

74. Special Function Databook, National Semiconductor, 1976.

75. Drexhage, K.H., "Structure and Properties of Laser Dyes," in Dye Lasers, F.P. Schafer, editor (Springer Verlag, 1973), Chapter 4, p. 161.

76. Webb, J.P., McColgin, W.C., Peterson, O.G., Stockman, D.L. and Eberly, J.H., "Intersystem Crossing Rate and Triplet State Lifetime for a Lasing Dye," Journal of Chemical Physics $\underline{5} 3,4227$ (1976). 
77. Pappalarde, R., Samuelson, H., and Lempicki, A., "Long Pulse Laser Emission from Rhodamine 6G Using Cyclooctatetraene," App. Phys. Lett. 16, 267 (1970).

78. Snavely, B.B. and Schafer, F.P., "Feasibility of CW Operation of Dye Lasers," Physics Letters 28A, 728 (1969).

79. Mar1ing, J.B., Wood, L.L., and Gregg, D.W., "Long Pulse Dye Laser Emission Across the Visible Spectrum," IEEE Journal of Quantum Electronics QE-8, 498 (1972).

80. This was roughly the minimum voltage found necessary to fire a 3 inch long, 1 atmosphere, argon filled lamp.

81. Gusinaw, M.A., "Effective Blackbody Temperature of High Current Gas-Filled Flashlamps," J. App. Phys. 44, 4567 (1973).

82. Private Communication, Condenser Products, Brooksville, Florida.

83. Rao, N.N., Basic Electromagnetics With Applications, (PrenticeHa11, Inc., N.J., 1972), p. 382.

84. These dimensions were chosen because they approximate the dimensions used in the dye laser.

85. Holzrichter, J.F. and Schawlow, A.L., "Design and Analysis of Flashlamp Systems for Pumping Organic Dye Lasers," Annals New York Academy of Sciences 168, 703 (1970).

86. Markiewicz, J.P. and Emmett, J.L., "Design of Flashlamp Driving Circuits," IEEE JQE QE-2, 707 (1966).

87. ILC Technology Air Cooled Flashlamp Catalog.

88. Ross, D., Lasers, Light Amplifiers and Oscillators, (Academic Press, 1969), p. 427 .

89. American Institute of Physics Handbook, (American Institute of Physics, 1976).

90. Yariv, A., Introduction to Optical Electronics, (Holt, Rinehart and Winston, 1971), p. 61.

91. Diffraction Grating Handbook, published by Bausch and Lomb (1970), - P. 24.

92. Loth, C., and Meyer, Y.H., "Study of a 1-Watt Repetitive Dye Laser," App. Opt.

93. Mack, M.E., "O.2-W Repetitively Pulsed Flashlamp-Pumped Dye Laser," App. Phys. Lett. 19, p. 108 (1971). 
94. Klein, M.V., Optics, (John Wiley and Sons, Inc., New York, $1970)$, p. $5 \overline{7} 1$.

95. Bourn's Optical Glass Catalog, Bourn's Optical Glass, 1200 Columbia Avenue, Riverside, California, 92507.

96. Pilloff, H.S., "Sinultaneous Two Wavelength Selection in the $\mathrm{N}_{2}$ Laser Pumped Dye Laser,", App. Phys. Let. 21, 339 (1972).

97. Beasly, J.D., "Electrooptic Laser Scanner for TV Projection Display," Applied Optics, 10, p. 1934 (1971).

98. Zernike, F.; "Refractive Indices of Ammonium Dihydrogen Phosphate and Potassium Dihydrogen Phosphate Between $2000 \AA$ and $1.5 \mu^{\prime \prime}$ J.o.s.A., 544, 1215 (1964)

99. Quantum Technology Ltd., Data Sheet 非:04, Quantum Technology, Toronto, Ontario, Canada.

300. Phillips, R.A., "Temperature Variation of the Index of Refraction of ADP, KDP, and Deuterated KDP," J.0.S.A., 56, 629 (1966).

101. Quantum Technology Data Sheet $\$$ F 704, Quantum Technology, Toronto, Ontario, Canada.

102. USM Corporation, Wakefield, Massachusetts.

103. Boyd, G.D. and Kleinman, D.A., "Parametric Interaction of Focussed Gaussian Light Beams," J.:App. Phys. 39, 3597 (1968). 
PROOF THAT THE MINIMUM SIZED BEAM WHICH CAN BE SYMETRICALLY PROPAGATED BETWEEN TWO POINTS IS ONE WHICH IS CONFOCALLY FOCUSSED In Chapter II it was asserted that the smallest beam which could be symetrically propagated between two points a distance L apart, was one whose beam radius w at the two ends of the path of propagation $z=0$ and $z=L$, was related to the beam radius $w_{0}$ at the path midpoint $z=L / 2$, by:

$$
w=w_{0} \sqrt{2}
$$

In this appendix we shall show that this assertion is in fact true.

The question can be posed simply as, "For a given path $\mathrm{L}$, what is the minimum value for the beam radius at the ends of the path?" Kogelnik and $\mathrm{Li}$ showed that the beam radius $w$ at the ends of the path was related to the pathlength $z$, the wavelength of the light and the radius of the beam in the waist region by:

$$
w=w_{0}\left[1+\left(\frac{\lambda z}{\pi w_{0}^{2}}\right)^{2}\right]^{1 / 2}
$$

The maxima and minima of this function can be determined by taking the derivative of (A1.2) with respect to $\mathrm{w}_{0}$, and setting that derivative equal to zero. Taking the derivative we get:

$$
\frac{d w}{d w_{0}}=\left[1+\left(\frac{\lambda z}{\pi w_{0}^{2}}\right)^{2}\right]^{1 / 2}-2\left(\frac{\lambda z}{\pi w_{0}^{2}}\right)^{2}\left[1+\left(\frac{\lambda z}{\pi w_{0}^{2}}\right)^{2}\right]^{-1 / 2}
$$

This can be reduced to

$$
\frac{\lambda z}{\pi w_{0}^{2}}=1
$$

a result which when substituted back into (A1.2) yields

$$
w=w_{0} \sqrt{2}
$$


which is the case of confocal focussing as was asserted in Chapter II. 